UNIVERSITÉ DU QUÉBEC

\author{
MÉMOIRE \\ PRÉSENTÉ A \\ L'UNIVERSITÉ DU QUÉBEC A CHICOUTIMI \\ COMME EXIGENCE PARTIELLE \\ DE LA MAITTRISE EN ÉDUCATION (M.A.)
}

PAR

FRANCE CLOUTIER

BACHELIĖRE EN ÉDUCATION

\begin{abstract}
Étude descriptive des caractéristiques des stades graphiques d'un groupe d'enfants de 11-12 ans ayant accès à un atelier de peinture dans la classe.
\end{abstract}




\section{Bibliothèque}

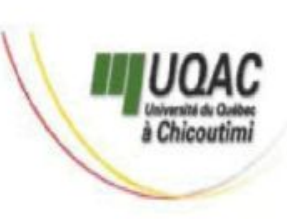

\section{Mise en garde/Advice}

Afin de rendre accessible au plus Motivated by a desire to make the grand nombre le résultat des results of its graduate students' travaux de recherche menés par ses research accessible to all, and in étudiants gradués et dans l'esprit des accordance with the rules règles qui régissent le dépôt et la governing the acceptation and diffusion des mémoires et thèses diffusion of dissertations and produits dans cette Institution, theses in this Institution, the I'Université du Québec à Université du Québec à Chicoutimi (UQAC) est fière de Chicoutimi (UQAC) is proud to rendre accessible une version make a complete version of this complète et gratuite de cette œuvre. work available at no cost to the reader.

L'auteur conserve néanmoins la The author retains ownership of the propriété du droit d'auteur qui copyright of this dissertation or protège ce mémoire ou cette thèse. thesis. Neither the dissertation or Ni le mémoire ou la thèse ni des thesis, nor substantial extracts from extraits substantiels de ceux-ci ne it, may be printed or otherwise peuvent être imprimés ou autrement reproduced without the author's reproduits sans son autorisation. permission. 


\section{RÉSUMÉ}

Cette étude a été réalisée entre novembre 1987 et mai 1988 auprès d'un groupe de 26 enfants de 11-12 ans dans une classe de sixième année. L'étude a pour objectif, d'une part, d'identifier où se situent les enfants de 11-12 ans dans l'acquisition des stades graphiques et de constater quels sont les traits distinctifs qu'ils utilisent pour constituer une image. D'autre part, l'auteure désire montrer qu'une organisation matérielle adéquate facilite l'accès constant à l'atelier de peinture en classe.

Le premier chapitre pose la problématique et définit le cadre conceptuel selon deux pôles. Le premier pôle identifie les stades du développement graphique à partir des recherches réalisées par Lowenfeld, Stern, Luquet et Ozinga. Le second présente l'importance de l'organisation matérielle de l'espace et plus précisément l'importance de l'aménagement physique d'un atelier de peinture.

Le deuxième chapitre présente une description de l'organisation matérielle et des modalités de fonctionnement de l'atelier de peinture privilégié pour l'expérimentation de l'étude. Les grilles d'analyse utilisées pour la lecture des peintures de ce groupe d'enfants sont élaborées à partir des recherches des quatre auteurs cités précédemment.

Les résultats présentés au chapitre III font ressortir l'apparition des caractéristiques des stades graphiques des pré-adolescents. Ces caractéristiques sont notées à partir de la représentation du personnage, de l'animal, du soleil, du nuage, de l'arc-en-ciel, de la maison, de l'arbre, de l'utilisation de la couleur et de l'organisation de l'espace feuille. La discussion tente de comprendre, d'expliquer l'utilisation des traits distinctifs et l'évolution des stades graphiques chez ce groupe d'enfants. La discussion propose également des pistes de travail pour une recherche ultérieure. Les observations reliées à l'organisation matérielle utilisée pour l'expérience font valoir que ce type d'aménagement physique permet l'accès régulier à un atelier de peinture en classe. 


\section{REMERCIEMENTS}

Je désire exprimer ma reconnaissance à ma directrice de mémoire, Madame Jacqueline Thériault, Ph.D., professeure-chercheure au département des Sciences de l'éducation de l'Université du Québec à Chicoutimi, pour la relation d'aide si précieuse qu'elle a su établir tout au long de cette étude, sa disponibilité et son soutien ont permis la réalisation de ce mémoire et le développement d'attitudes de recherche.

Je remercie Manon Doucet, M.A., pour les conseils judicieux relatifs à l'élaboration des grilles d'analyse et pour les petits mots complices qui aident à progresser.

Un merci affectueux à Auguste Cloutier qui a su semer en moi la curiosité, qui a su bricoler avec moi; à Henriette Trudeau pour ses encouragements persuasifs; à Alain Collard pour la reproduction en diapositives des peintures et le soutien constant sans lequel ce projet n'aurait pu se réaliser.

Un merci spécial à Johanne Beaumont pour son travail remarquable de dactylographie.

Enfin, je remercie la Commission scolaire Valin, en particulier Andrée Barrette, pour le support financier. 


\section{TABLE DES MATIÈRES}

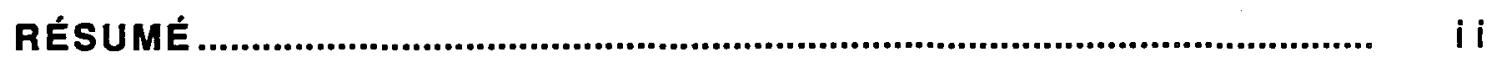

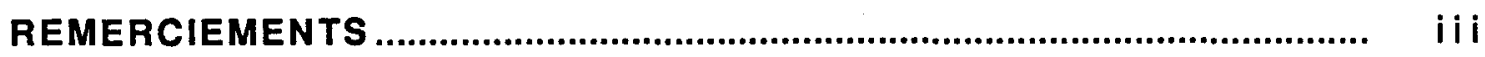

TABLE DES MATIĖRES ...................................................................... iv

LISTE DES TABLEAUX ...................................................................... vi

LISTE DES FIGURES.............................................................................. viii

LISTE DES ILLUSTRATIONS .................................................................... ix

INTRODUCTION ........................................................................................

CHAPITRE PREMIER: La problématique et le cadre conceptuel............... • 4

La problématique ............................................................................................... 5

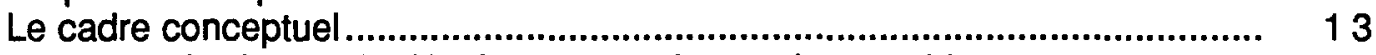

Les études sur le développement des stades graphiques.................... 15

Le stade du gribouillage ou début de l'expression de soi (2-

4 ans)

Le stade pré-schématique ou premier essai de représentation

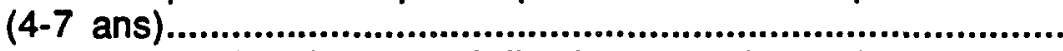

Le stade schématique ou réalisation de la forme (7-9 ans).....

Le stade du réalisme visuel ou âge du phénomène de bande (9-

12 ans)

Le stade du pseudo-naturalisme ou âge du raisonnement (12-

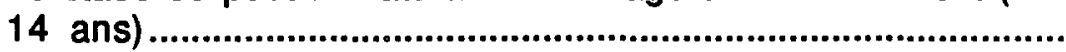

Les études sur l'importance de l'organisation matérielle de l'espace

CHAPITRE II: Méthodologie ................................................................... 50

Les sujets ...................................................................................... 51

L'organisation matérielle de l'atelier de peinture ........................................ 51

La procédure ............................................................................................. 54

Les modalités de fonctionnement ..................................................... 54

La technique et l'utilisation du matériel............................................. 55

Le plan d'analyse des données ............................................................. 56 
CHAPITRE III: Description et discussion des résultats..............................

Les résultats relatifs a l'apparition des caractéristiques des stades graphiques des pré-adolescents.

Les résultats de la représentation du personnage..............................

Les résultats de la représentation de l'animal.

Les résultats de la représentation du soleil, du nuage et de l'arcen-ciel

Les résultats de la représentation de la maison .................................. 75

Les résultats de la représentation de l'arbre..................................... 77

Les résultats de l'utilisation de la couleur.......................................... 79

Les résultats de l'organisation de l'espace feuille............................ 81

Discussion

85

Les observations reliées à l'organisation matérielle............................. 96

Conclusion

RÉFÉRENCES

100

\section{ANNEXES}

Annexe 1 Tableau-synthèse des stades graphiques selon Lowenfeld............ 104

Annexe 2 Modèles de schémas proposés par A. Stern................................ 110

Annexe 3 Manifestations idéographiques proposées par Clasca Ozinga........ 126

Annexe 4 Reproduction du modèle table-palette de Stern adapté aux réalités d'une classe........................................................................... 128

Annexe 5 Reproduction du modèle de rangement pour la conservation des peintures.

Annexe 6 Reproduction des grilles utilisées pour l'analyse des peintures .. 132

Annexe 7 Le nombre de productions réalisées par chacun des pré-adolescents 


\section{LISTE DES TABLEAUX}

Tableau

$1 \quad$ Nombre de traits distinctifs du stade pré-schématique présents dans les personnages des enfants de 11-12 ans

2 Nombre de traits distinctifs du stade schématique présents dans les personnages des enfants de 11-12 ans

3 Nombre de traits distinctifs relatifs à la manière de réaliser le personnage

$4 \quad$ Variété et nombre de traits distinctifs présents dans la représentation de l'animal chez des enfants de 11-12 ans.

5 Variété et nombre de traits distinctifs du stade schématique présents dans la représentation de l'animal chez des enfants de 11-12 ans......

6 Variété et nombre de traits distinctifs du stade réalisme visuel présents dans la représentation de l'animal chez des enfants de 11-12 ans

$7 \quad$ Variété et nombre de traits distinctifs du stade pseudo-naturaliste présents dans la représentation de l'animal chez des enfants de 1112 ans.

8 Représentation de la forme soleil

9 Représentation de la forme nuage.

10 Représentation de la forme maison.....

11 Représentation de la forme arbre

12 Utilisation de chacune des couleurs 
13 Le nombre de couleurs utilisées dans chacune des peintures.............. 80

14 Utilisation de la couleur par rapport aux quatre traits distinctifs.... 80

15 Utilisation de la couleur par rapport aux six traits distinctifs........... 81

16 Utilisation des éléments composant l'organisation de l'espace au stade pré-schématique................................................................................

17 Utilisation des éléments composant l'organisation de l'espace au sta-

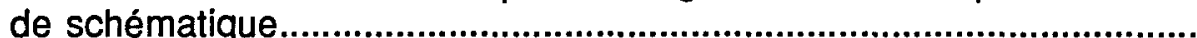

18 Utilisation des éléments composant l'organisation de l'espace au stade réalisme visuel. 


\section{LISTE DES FIGURES}

\section{Figure}

1 Nombre de traits distinctifs de la représentation du personnage pour les stades étudiés.................................................................................

2 Nombre de traits distinctifs de la représentation du personnage propres au stade de réalisme visuel.

3 Nombre de traits distinctifs de la représentation du personnage propres au stade pseudo-naturaliste.........................................................

4 Pourcentage de la variété des formes représentées dans l'ensemble

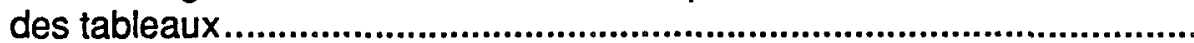




\section{LISTE DES ILLUSTRATIONS}

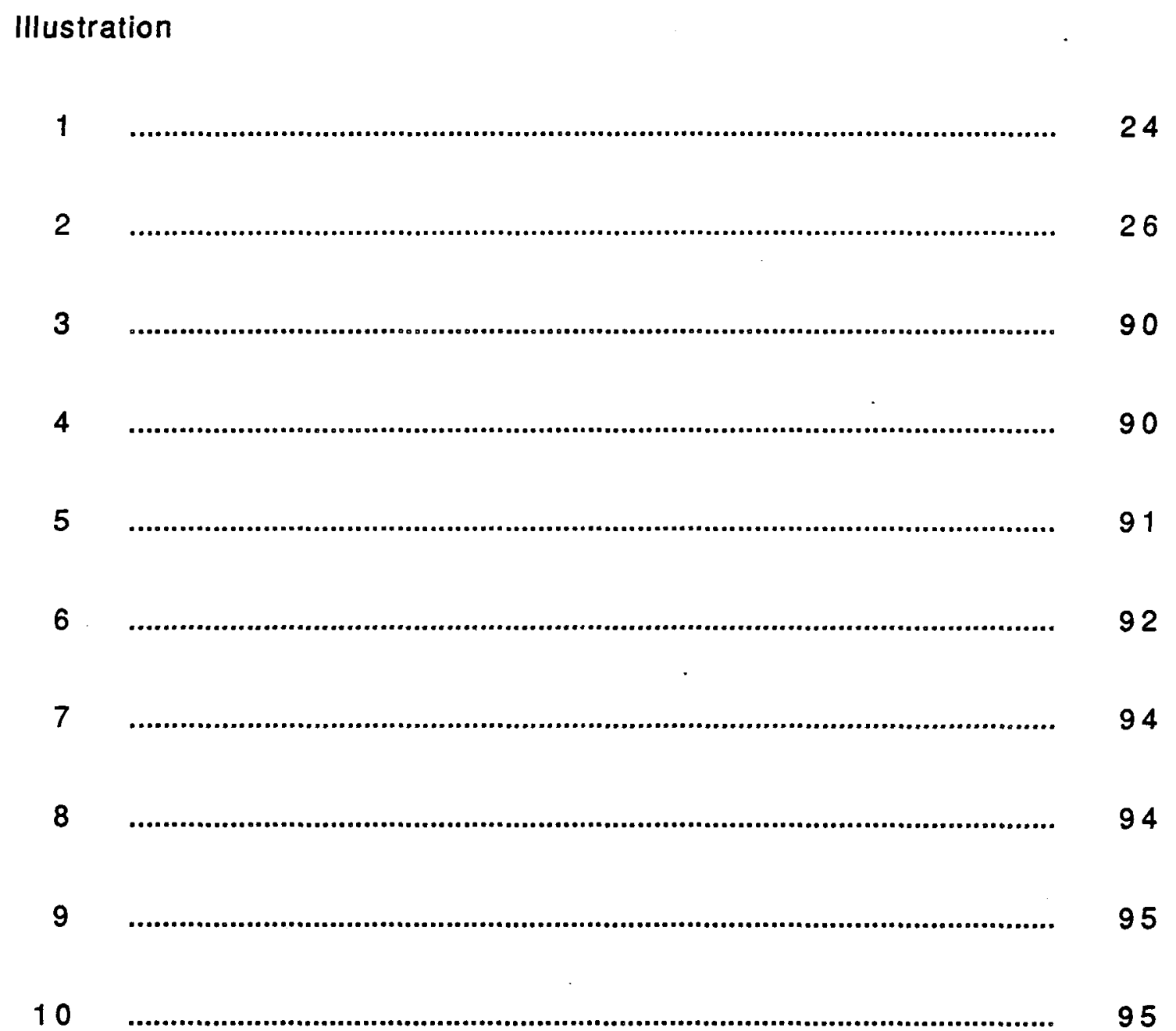


INTRODUCTION 
Le médium peinture est utilisé par l'individu pour exprimer les connaissances, les sentiments, les craintes, les espoirs. C'est un outil de communication qui s'est transformé au fil des temps; du message gravé sur la pierre au fond des cavernes, il est devenu affiche, aquarelle, gouache, huile, ..., qui habitent maintenant maisons, musées, édifices publics, ..., mais continue de témoigner des préoccupations de l'homme vers une meilleure compréhension de soi et du monde qui l'entoure.

Le jeune enfant saisit spontanément le crayon, le pinceau, afin d'exprimer ses émotions, ses espérances, ses souvenirs et ses interrogations. Notre organisation sociale ne privilégie pourtant pas les arts plastiques dans le développement de l'enfant. Elle axe plutôt les énergies sur un développement pratique, rentable, comptabilisable de l'individu.

Ce travail tente de clarifier une notion, celle de la construction du langage plastique chez les enfants à travers la peinture. Cette réflexion est avant tout limitée à des fins pédagogiques. Une dimension importante de la production artistique de l'enfant sera ignorée, soit l'aspect psychologique de la peinture d'enfant. II est quand même probable que par les thèmes étudiés, une orientation générale de la communication que veut organiser l'enfant pourra en être dégagée. Cette approche ne tient pas compte de l'importance du signifiant que chaque enfant révèle à travers ses productions, mais elle en reconnaît tout de même l'existence certaine. 
La présente étude cherche plutôt à offrir à l'enfant un aménagement de l'espace appelé atelier de peinture et à observer comment il traduit sa connaissance d'un langage plastique.

Ce rapport de recherche comprend trois parties. La première fait état de la problématique et du cadre conceptuel. Le deuxième chapitre élabore la méthodologie utilisée et présente le mode d'analyse des données. La dernière partie tient compte des résultats et est suivie d'une discussion. 


\title{
CHAPITRE PREMIER
}

\author{
La problématique \\ et le cadre conceptuel
}




\section{LA PROBLÉmATIQUE}

La classe maternelle est un milieu de vie aménagé en grande partie par l'adulte pour l'enfant de 5 ans, dans le but de "permettre à l'enfant de niveau préscolaire de . poursuivre son cheminement personnel, d'enrichir sa capacité d'entrer en relation avec les autres et celle d'interagir avec son environnement" (Programme d'éducation préscolaire, MEQ, 1981).

Afin de répondre à cet objectif global, le programme d'éducation préscolaire privilégie le fonctionnement en atelier, expliqué à travers les différents documents d'accompagnement mis à la disposition des intervenantes $^{1}$. Ce fonctionnement se traduit habituellement par un regroupement de deux à six enfants qui travaillent à une activité de même type avec un matériel, une organisation spécifique, et ce, dans une aire, un espace qui est régulièrement enrichi, aménagé de façon à susciter un intérêt continu. Un des facteurs caractérisant ce type de fonctionnement pédagogique . est l'importance accordée à l'organisation de l'environnement.

Kritchesky, Prescott et Walling (1969) soulignent que l'organisation de l'espace physique invite a la manifestation de certains comportements et que l'un des facteurs les plus importants de la qualité d'un programme est la richesse de l'envi- . ronnement. Pommerleau et Malcuit (1983), pour leur part, rappellent que "depuis

1 Tout au long de l'étude, le genre féminin est utilisé parce que la majorité du personnel cuvrant au primaire est féminin. 
plusieurs années, les chercheurs s'accordent pour reconnaître que l'environnement matériel et non pas seulement l'environnement social, a un effet sur le développement de l'enfant" (p.134). Selon eux, un environnement matériel varié et complexe . qui favorise la réactivité des objets disponibles affecte le comportement.

Le rôle de l'éducatrice à la maternelle est d'intervenir afin d'orienter l'enfant vers une plus grande maîtrise de son potentiel, vers l'acquisition de connaissances et d'habiletés permettant une appropriation de plus en plus raffinée et consciente de son espace-vie dans un cadre évolutif.

II s'agit, pour elle, de provoquer une réflexion, un questionnement, une action, un changement, de suggérer des problèmes, de favoriser leurs résolutions, de répondre à une interrogation, de stimuler un cheminement, de faire preuve de présence continue, de conscience des enjeux, de prospective, de communiquer le goût, le désir d'apprendre à reconnaître ce qui est bon pour soi dans le respect des règles de fonctionnement, dans la négociation des changements désirés, et cela, dans une perspective holistique. L'éducatrice n'exige pas son choix, sa préférence, ne dicte pas le conformisme ou la solution institutionnalisée. Elle propose des situations d'apprentissages signifiantes. Cette tendance dérive de la philosophie humaniste, des finalités que le MEQ privilégie à travers les nouveaux programmes et qui rejoignent les valeurs moins conformistes et plus individualistes de la société actuelle.

Ce type d'organisation pédagogique se concrétise entre autres, par le choix de l'aménagement physique, par les différentes injections de matériel effectuées en cours d'année, par la planification et la distribution des outils dans le temps. "II 
semble que le materiel offert à l'enfant, et davantage encore l'organisation de ce . matériel dans les aires et les ateliers, ont une influence sur la qualité du jeu" (Thériault et al., 1987b).

Parmi les organisations matérielles hautement privilégiées par les enfants de . 5 ans, il y a l'atelier de peinture. L'enfant y est naturellement invité, par la magie du pinceau et de la couleur, à exprimer des formes. Il a donc ainsi la possibilité de raconter ses préoccupations, de raffiner son geste, de cheminer dans le monde du symbole.

Ce moyen d'expression est, pour l'adulte qui l'accompagne, une forme de lec- . ture de l'image intérieure, de l'image-mot que l'enfant se fait progressivement de la réalité. C'est ainsi que nous retrouvons dans presque toutes les classes de maternelle un coin, un espace réservé à la peinture, et ce, avec un équipement de base varié.

A partir d'une analyse du matériel utilisé dans les classes maternelles de la région du Saguenay-Lac-St-Jean, on a répertorié différents modèles d'organisation d'atelier peinture pouvant se regrouper autour de quatre types à partir d'un équipement spécifique: la table, le chevalet pliant, le mur avec un support au bas de la surface de travail et l'utilisation du mur avec la table-palette de Stern présentant une gamme de couleurs (Thériault et al., 1987d).

Le modèle table propose des pots de gouache, des pots d'eau, des pinceaux et des feuilles. Les enfants s'assoient autour de cette table et partagent le matériel. Le modèle chevalet pliant permet de placer un choix de-six à huit couleurs sous la 
feuille de l'enfant. Après avoir fixé sa feuille, il effectue son travail debout en même temps qu'un autre enfant placé en face de lui, mais qu'il ne voit pas. Le modèle au mur offre un panneau au mur couvrant un espace de trois pieds de hauteur et d'environ dix pieds de longueur et ce, à deux ou trois pieds du sol, sur lequel l'enfant pose sa feuille. Les pots de peinture sont plaçés sous la feuille et quatre ou cinq enfants peuvent réaliser un travail simultanément. Chaque enfant bénéficie d'un choix de cinq à sept couleurs de gouache et d'un choix de pinceaux correspondants. Ils travaillent côte à côte. Le modèle mur et table-palette de Stern propose un mur recouvert de haut en bas de "tintes" ou d'un autre matériau équivalent sur lequel l'enfant pique sa feuille. Une longue table située à quelques pieds de ce mur contient huit, dix, 18 godets gouache et parallèlement huit, dix, 18 godets eau. II y a un seul pinceau correspondant à chacune des couleurs et chaque pinceau a la même dimension, soit trois-quart de pouce. Les couleurs et les pinceaux sont toujours disposés dans le même ordre, au même endroit sur la table.

Après l'analyse de productions d'enfants évoluant dans chacun des modèles cités, selon une grille basée sur les stades graphiques établis par Lowenfeld, Stern et Luquet, les résultats portent à croire qu'une adaptation du modèle mur et table-palette de Stern favoriserait davantage la manifestation d'un plus grand nombre d'éléments constitutifs de chacun des critères d'analyse et, par conséquent, aurait une influence sur l'apparition des stades graphiques propres à l'enfant de cinq ans (Thériault et al., 1987d).

Cet atelier peinture, pourtant primordial au préscolaire, perd de sa force au primaire, au profit d'autres disciplines. L'échelle des valeurs éducatives semble 
modifiée et l'atelier peinture, évidemment nécessaire à l'entrée en institution scolaire, disparait à peu de chose près, faute de temps, faute de formation adéquate chez les enseignantes, faute d'organisation matérielle. Cette activité semble perdre, tout au long de l'élémentaire, le signifiant qu'elle avait pour l'enfant qui ne voit plus un grand intérêt à ce moyen d'expression.

Le programme des Arts Plastiques au primaire (1981) poursuit pourtant un • objectif global compatible avec celui du préscolaire, soit "d'amener l'enfant à faire et à voir son image à chaque étape de son évolution graphique pour acquérir une connaissance intuitive de lui-même et de son environnement" (p.48).

Ce programme soutient également que "c'est dans la mesure où l'individu est conscient d'être un être vivant, sensible, unique et créateur qu'il peut saisir son rôle dans la société et contribuer à son évolution" (p.47).

Or, très peu d'enfants de $6^{\theta}$ année jouissent d'environnement leur permettant de développer des habiletés graphiques malgré les énoncés émis dans ce programme, puisque l'atelier peinture est absent chez les 11-12 ans en milieu scolaire au Québec.

Un enfant placé dans un lieu de fọtune reçoit le message subtil que l'environnement n'a pas d'importance. II faut organiser l'espace de manière à offrir une variété dans les objets offerts, leur accessibilité, leur mode de rangement simplifié et leur classification claire (Goetz et Allen, 1983). 
Ces pré-adolescents ont donc jusqu'ici été très peu encouragés dans le domaine de la peinture. Luquet affirme que "la plupart des enfants ont perdu tout plaisir au dessin, précisément parce qu'ils se sont rendu compte que dans ce domaine, ils ne sauraient de toute façon rien produire de valable. Ce fait n'est presque jamais mis en doute". II poursuit en disant "que cette situation proviendrait du mauvais enseignement qui ne répond pas aux aspirations légitimes de l'enfant, celui-ci est découragé . d'arriver au réalisme visuel".

Parmi les études réalisées au Québec et voisinant la présente étude dans le domaine des Arts Plastiques au primaire, le Répertoire de l'ensemble des recherches effectuées entre 1960 et 1980 en enseignement des Arts au Québec (1982) mentionne une seule étude sur "l'état actuel de l'enseignement des Arts Plastiques" menée par Wim Huysecom en 1976 à l'Université Concordia, ce mémoire de maîtrise révèle que seulement $28 \%$ des enfants, sur un échantillonage de 1500 garçons et filles de 11-12 ans, présentent l'expression du stade graphique correspondant à leur âge mental. La majorité des autres enfants s'inscrit encore dans la période du stade schématique, donc en retard sur leur âge mental. Au cours de cette étude, les enfants ont produit un seul dessin sur un thème précis, dessin qui devait présenter un personnage, un arbre et une maison. Cette production a été analysée à partir des carac- . téristiques du tableau synoptique de Lowenfeld concernant l'évolution graphique et tentait, entre autres, de vérifier si les enfants qui ont une évolution normale et qui ont suivi depuis quelques années des cours d'arts plastiques à l'école se situent à un stade d'évolution graphique qui correspond, à peu près, à leur évolution mentale. 
Les résultats ont soulevé de nouvelles questions, l'auteur a donc poursuivi l'é- tude en tentant de savoir s'il y a des différences dans les productions des enfants entre les écoles où l'enseignement des arts plastiques est assuré par un spécialiste et d'autres écoles où cet enseignement est donné par les titulaires.

Une nouvelle expérimentation fut donc reconduite auprès de 1200 élèves de 6, 8,11 et 12 ans. Les résultats ne montrent pas de différence pour le groupe de 6 ans si l'enseignement des arts plastiques est assuré par un généraliste ou un spécialiste. Le type d'enseignement donné influence cependant les productions plastiques des deux autres groupes. Règle générale, dans les écoles sans spécialiste, il y a progression dans les stades graphiques de 6 à 8 ans mais cette évolution se trouve arrêtée peu après. Dans les écoles comptant sur les services d'un spécialiste, la spontanéité progresse de façon normale de 6 à 12 ans.

L'auteur a, par la suite, suggéré que les enseignants généralistes soient assistés dans leur tâche en enseignement des arts plastiques par une personne-ressource compétente en la matière afin de remédier à cette faiblesse dans l'éducation des enfants. Cette étude ne tenait compte que d'une seule production pour asseoir ces recommandations et semble difficilement généralisable, quoique fort acceptable.

Différentes Commissions Scolaires du Québec, soit Baldwin-Cartier, Sherbrooke, Mont-Fort, Port-Royal ont, pour leur part, tenté des expériences afin de remédier à l'absence de spécialistes en arts, au manque d'activités susceptibles de stimuler l'évolution graphique des enfants et à l'absence d'activités de formation des maîtres. 
Ces expériences visaient plus précisément à sensibiliser à la pratique de l'enseignement des arts plastiques, à amener les généralistes à approfondir leur perception du rôle des arts dans le développement intégral de l'enfant, à développer une pédagogie basée sur l'expression libre et personnelle, à reconnaître l'art comme facteur de structuration de la pensée, à combler le besoin de perfectionnement ressenti par les généralistes, à simplifier l'utilisation du matériel, à élargir le répertoire des formes d'arts connues des élèves et l'éventail de leurs habiletés techniques.

La plupart de ces études ont été évaluées globalement. Les personnes impliquées dans ces expériences ont déploré le manque de suivi et le manque de temps alloué à la réalisation des projets. II semble que ces tentatives n'aient pas réussi à intégrer l'éducation artistique dans la vie quotidienne de l'école primaire, elles demeurent isolées, fragiles et facilement délogeables.

La majorité des recherches effectuées dans ce domaine jusqu'à maintenant sont souvent cloisonnées, c'est-à-dire expérimentées dans le cadre spécifique d'un atelier peinture ou hors du contexte scolaire habituel. L'atelier de peinture demeure un lieu auquel l'enfant accède durant son temps de loisir, il n'est pas intégré à l'organisation scolaire, il est accessoire à ce milieu. Par conséquent, seulement quelques jeunes privilégiés grandissent en poursuivant cette démarche artistique qu'est la peinture. Le développement du langage plastique à travers les stades qui le caractérisent est donc sous-stimulé chez l'ensemble des pré-adolescents à cause du peu de valeurs que nous y accordons comme société, des budgets et des espaces restreints offerts à chaque classe afin de créer un climat physique favorable et à cause d'une formation sommaire des enseignants dans ce domaine. 
L'expression par la peinture demeure pourtant, à cet åge, la manifestation d'un ensemble de signes, linventaire de sensations, de sentiments, de connaissances de l'image du moi qui peut devenir un moyen de connaissance de l'enfant pour l'adulte et d'équilibre intérieur pour l'enfant.

Cette étude a pour objectif, d'une part, d'observer si les éléments caractérisant . les stades graphiques des enfants de 11-12 ans se retrouvent dans les productions d'un groupe d'enfants de cet âge, et d'autre part, d'évaluer le rôle que joue dans la classe l'organisation matérielle d'un atelier de peinture permanent et facilement accessible.

\section{LE CADRE CONCEPTUEL}

Le cadre conceptuel s'appuie d'abord sur la recension des écrits concernant les stades graphiques qui constituent le développement du langage plastique chez l'enfant et, par la suite, sur les écrits démontrant l'importance qu'a l'environnement matériel sur le développement de l'enfant.

Lowenfeld (1970), Luquet (1967), Stern (1963a, 1963b) et Ozinga (1969) * sont les auteurs qui ont travaillé le langage plastique et les stades graphiques qui le caractérisent comme pouvant être réinvestissabile en pédagogie, avec l'oeil du practicien plutôt que du clinicien. En effet, ils ont étudié les manifestations graphiques de l'enfant en relation avec le développement. 
Malgré leurs écrits nuancés, ils ont repéré des stades graphiques plus ou moins reliés à l'âge de l'enfant, des périodes de manifestations graphiques associables à la construction du monde que se fait ce dernier, à la connaissance qu'il en retient. Ils ont construit des échelles graduées illustrant l'évolution du mode d'appropriation que fait l'enfant par la ligne, par la représentation d'objets animés et inanimés, par l'utilisation de la couleur et par l'organisation de l'espace.

Lowenfeld situe le développement du langage plastique à travers des stades gra- . phiques correspondant plus ou moins à six groupes d'âge. Pour lui, le langage se construit du simple au complexe. Le gribouillage, cette tentative involontaire et maladroite, prépare l'apparition de la représentation de la forme, d'abord dessinée sans ordre dans l'espace, puis précisée à travers une organisation partielle. La re- . présentation de la forme se positionne ainsi dans un espace de plus en plus défini, ordonné, correspondant à des manifestations comprises dans les stades ultérieurs.

Luquet attache l'étiquette de conquête du réalisme à la démarche graphique. D'un réalisme qui se frotte à l'imaginaire, le réalisme fortuit se précise ensuite, gagne en consistance pour rejoindre le réalisme adulte.

Stern définit, pour sa part, la formation de ce langage à travers le raffinement . des formes de base: personnage, maison, arbre, animal, et également à travers la croissance de l'organisation spatiale et la représentation de plus en plus objective de la couleur. II explique ce langage comme une grammaire, c'est-à-dire la construction de structures logiques qui se précisent, se juxtaposent, se complexifient. 
Clasca Ozinga soumet et définit des caractéristiques concrètes de représentations graphiques qu'utilise l'enfant afin de développer son langage plastique.

Les écrits que chacun de ces auteurs nous proposent présentent des ressemblances par le fond mais sont construits d'un oeil différent. Ils sont élaborés dans les pages qui suivent afin de préciser leur point de vue respectif dans la compréhension du développement du langage plastique.

\section{Les études sur le développement des stades graphiques}

Lowenfeld $(1968,1969,1970)$ affirme que l'expression artistique joue un - rôle vital dans l'éducation. Dessiner et peindre sont des gestes complexes par lesquels l'enfant rassemble des éléments de son expérience afin de représenter un nouveau signifiant. L'enfant choisit ces éléments, les interprète, les représente et nous livre ainsi sa pensée, ses sentiments, sa vision du monde, c'est une activité qui l'engage totalement.

L'importance de l'activité créatrice dans la compréhension de la croissance se situe au plan émotif, à travers la flexibilité des modèles; au plan intellectuel, à travers l'évolution de la représentation des formes; au plan physique, dans le contrôle du geste; au plan perceptuel, dans l'habileté à apprendre qui dépend de la qualité et du sens des expériences sensorielles; au plan social, dans l'identification de soi et des autres; au plan esthétique, dans le sens d'organisation et d'ordre et au plan créateur, dans le sens de fluidité ou de liberté émotive. 
À la lecture de Lowenfeld, c'est la construction d'une personnalité que l'on envisage; il est convaincu que l'expression artistique contribue à la formation de la personnalité parce qu'elle présente une confrontation avec soi-même et son expérience. Le besoin d'identité étant primordial, il s'agit de permettre le développement " de la sensibilité à l'environnement, à ses besoins et à ceux des autres à travers le percevoir, le faire et le voir par la compréhension des connaissances et leur utilisation en habileté. La perception des objets et des êtres dans l'environnement se * structure en image intérieure, se dégage en image représentée que l'enfant peut discuter. Le développement de cette sensibilité favoriserait l'ouverture d'esprit, la flexibilité, la fluidité et l'autonomie.

It is the flexibility of expression wich has a decisive influence on his emotional growth and his total personality (Lowenfeld, 1969, p.88).

L'enfant qui demeure ouvert à son expérience se construit, développe l'habileté à chercher la réponse au lieu de l'attendre. II manifeste sa présence, son identité dans une démarche dynamique, dans un contexte de prise en charge, d'autonomie élargissant ainsi son cadre de référence.

II se structure dans sa logique, consolide ses acquis, travaille à l'identification et l'expression de soi à travers des apprentissages signifiants. Chaque manifestation artistique est le reflet d'une étape dans le long processus du développement de la pensée et procure richesse et équilibre intérieur. 
L'enfant, sensible à son expérience, à ses besoins et attentif à sa démarche, peint une relation signifiante qui colle à ses préoccupations et démontre l'importance qu'a l'objet ou la personne dans sa relation. II décrit ainsi la relation entre son ex- . périence et son expression créatrice.

Lowenfeld insiste sur l'interdépendance entre développement, croissance et création, gage de confiance en soi, de communication, de formation d'attitudes positives.

Lowenfeld a identifié différents stades graphiques associés à l'organisation in- . terne, à l'intégration que l'enfant exprime, à la perception qu'il a de ce qui l'entoure. II regroupe ces représentations en six étapes qui sont interreliées. Ces stades sont compris par le biais des préoccupations maintenant classiques de chaque âge de l'enfance. Pour le besoin de l'étude, cinq de ces stades sont expliqués.

\section{Le stade du griboullage ou début de l'expression de sol (2-4 ans)}

C'est à cette période que l'enfant commence à s'approprier ses premiers modèles d'apprentissage. Il dégage alors une image de lui qui deviendra déterminante tout au long de sa vie. La qualité des mécanismes de perception qu'il développe compose . une structure fondamentale. Ils constituent la rampe de lancement de l'acquisition des stades graphiques et doivent être reçus avec toute l'importance qu'une base requiert parce qu'ils sont aussi une avenue supplémentaire de communication pour l'enfant, une expérience de confiance en soi à travers la maîtrise de la ligne. II divi- 
se ce stade en trois étapes: le gribouillage en désordre, le gribouillage contrôlé et le gribouillage identifié.

Le gribouillage en désordre est l'exploration du geste dans toutes les directions, l'oeil n'étant parfois même pas complice de ce mouvement. C'est la découverte de préhension du crayon, la découverte de l'ampleur du mouvement de l'avant-bras, l'expérience kinesthésique sans intention, ni représentation.

Le griboullage contrólé est la découverte par l'enfant de la relation entre le geste et la marque. II établit le contrôle visuel sur la trace, varie la ligne à l'horizontale, à la verticale, en cercle, droite, sinueuse, angulaire puis répète ce geste afin de le contrôler. Ce sont là ses premières tendances à exprimer le rythme, l'apprentissage de la coordination entre l'oeil et le mouvement. Ces découvertes l'amènent à poursuivre sa recherche dans l'essai, la variété, le choix de différentes couleurs, le remplissage de la page. II emploie alors la couleur pour signifier la relation entre les formes.

Par le griboullage identlflé, l'enfant manifeste maintenant une intention à travers la description verbale. La marque est devenue moyen de communication, de . la pensée kinesthésique il passe à la pensée imaginative. Il équilibre sa présence sur la feuille, se décrit à lui-même ce qu'il réalise. Sa description demeure méconnaissable pour l'adulte, ne colle pas à la représentation réelle, ce qui est en jeu c'est l'intention qu'il accorde à la ligne qui peut être une échelle au début de l'activité et une glissade à la fin, c'est la représentation au présent de la relation qu'il établit avec l'environnement qui motive son action avant tout. 


\section{Le stade pré-schématique ou premier essal de représentation (4-7 ans)}

A ce stade, l'enfant dessine différemment, il devient plus conscient des formes qui l'entourent, de sa relation dans le monde. II traduit ce qui a de l'importance pour lui et comment il organise sa relation avec l'environnement, sa façon de le penser, de le construire. II représente ce qu'il perçoit, cette expérience qu'il construit en prenant conscience de l'environnement est par la suite déterminante sur le développement de ses attitudes coopérantes. Cette période permet le raffinement et l'ajustement de la coordination visuelle. Un changement dans sa production est un changement dans ses sensations, ses perceptions et sa pensée.

Afin d'organiser sa production picturale, l'enfant travaille à partir de trois plans ou éléments de composition. Ce sont: la représentation de la forme, l'utilisation de la couleur et l'organisation de l'espace. Au fur et à mesure qu'il grandit, ce sont ces aspects de la composition qui évoluent. Chacun des stades ultérieurs est donc analysé en fonction de ces trois critères.

\section{La représentation de la forme.}

La représentation de la forme, c'est-à-dire d'un objet animé (personnage ou . animal) ou inanimé (objet de l'environnement), est l'évolution d'une série de lignes vers la représentation, la définition, la reconnaissance de formes et la communication graphique. Le personnage en est la première manifestation, il présente une tête . et des jambes, puis l'enfant lui ajoute des bras et enfin un corps. 
Ces formes deviennent fonctionnelles et sont ce que l'enfant sait des choses, seIon la valeur qu'elles ont pour lui, la concrétisation de sensations qu'il juge importantes. Son personnage, sa maison traduisent sa recherche de nouveaux concepts, ils représentent des ajustements et des modifications constantes.

Vers 5 ans, on distingue dans son travail, un personnage, une maison, un * arbre. Vers 6 ans, ces éléments sont reconnaissables et dessinés en relation les uns avec les autres, ce n'est plus une maison, mais la maison du personnage, puis à 7 ans, ces représentations de la forme se stabilisent.

\section{L'utilisation de la couleur.}

Le souci de la forme domine celui de la couleur. L'enfant utilise la couleur pour établir une relation entre ses formes, sans lien avec la réalité mais plutôt à travers un échange émotif, une organisation harmonieuse de sa page.

\section{L'organisation de l'espace.}

Lorsque l'enfant dessine un personnage, il place les objets autour de lui. Ces. objets peuvent être au-dessus, en-dessous, à côté les uns des autres et sans relation spatiale entre eux sur la feuille. Ils sont rendus selon la compréhension qu'il en a. II ne se peint pas avec, mais bien à côté des objets selon l'ordre qui surgit dans sa tête. II n'a pas établi de relation spatiale en dehors de lui, tout est lié au concept qu'il a . de lui-même. C'est l'époque du "body space". 
Ce stade est donc caractérisé par la flexibilité et le changement dans la représentation des concepts. L'enfant exagère ce qu'il perçoit, changeant ainsi son mode de représentätion et la représentation qu'il a de lui.

\section{Le stade schématique ou réalisation de la forme (7.9 ans)}

L'enfant arrive maintenant à un concept défini du personnage, de l'animal et de l'environnement et le traduit par une connaissance active et consciente de l'objet représenté. C'est pour lui le moment de renforcer ses formes en répétant les figures, en les intégrant, en consolidant les acquis. II change ou transforme l'objet seulement s'il y a signifiance, si un fait ou une situation lui a permis d'en connaître davantage les propriétés et donc d'en exprimer la substance.

\section{La représentation de la forme.}

A 7 ans, le personnage dessiné présente tête, bras, corps, jambes, mains différenciées, doigts, pieds différenciés, cou, cheveux, yeux différents du nez et parfois vêtements. II est vu de face par certains, de face et profil par d'autres. Son corps, ses jambes, ses bras, ses vêtements peuvent être un cercle, un triangle, un carré, un ovale ou un rectangle. Ce personnage est devenu le reflet du développement de l'enfant, de son individualité et est la combinaison de plusieurs facteurs: le proces- * sus de pensée, la sensibilité aux sensations et lé développement de la perception.

\section{L'utilisation de la couleur.}

Au stade schématique, le jeune peintre découvre la relation de l'objet et de la * couleur, catégorise, fait des regroupements et généralise: le ciel est bleu, l'herbe est 
verte. II trouve un ordre logique dans le monde et établit la relation concrète avec les choses qui l'entourent, il reproduit les objets en relation les uns avec les autres. Les règles et les normes font donc partie de ses sujets privilégiés. La première re- lation signifiante qu'a un enfant avec un objet détermine l'utilisation de la couleur qu'il en fait et se développe dans la répétition, l'équilibre et la spontanéité. II utilise les couleurs sans variante, dessinant ainsi un ciel bleu malgré la pluie.

\section{L'organisation de l'espace.}

L'enfant prend conscience de l'ordre et de la relation spatiale qu'il traduit par la ligne de sol sur laquelle il dépoșe toutes ses formes. Cette ligne peut être plan- , cher, rue, base sur laquelle les objets sont posés, debout un après l'autre.

En découvrant le sens de la ligne droite ou courbe, l'enfant explorera deux types de lignes de sol: une au bas de la feuille et une ligne de ciel en haut de la page. II explorera également l'exagération de l'objet, le rabattement, l'espace entre le sol et le ciel. II démontrera ainsi son expérience subjective de l'espace par le mélange plan-élévation réglant son souci de montrer tout ce qu'il veut et sait. La présence du rabattement annonce la fin du "seesaw itself".

A ce stade, en plus des éléments de représentation de la forme, l'utilisation de . la couleur et l'organisation de l'espace, s'ajoute l'élément temps-espace qui présente des caractéristiques propres à cette période et qui disparaîtront dans les stades ultérieurs. 


\section{Le temps-espace.}

Cette notion de temps-espace permet à l'enfant d'exprimer différentes sé- . quences d'un même endroit sur la même feuille ou différentes actions placées dans des temps différents. Cette représentation spatio-temporelle surgit avec l'urgence de communication et l'importance de l'action. A titre d'exemple, l'enfant dessine à la fois l'intérieur et l'extérieur d'une maison, en utilisant la transparence, afin de démontrer une situation qui ne peut être vue en même temps mais qui le préoccupe sous ces deux aspects.

\section{Le stade du réalisme visuel ou âge du phénomene de bande (9-12 ans)}

L'expérience de groupe prend à ce stade, beaucoup d'importance. L'enfant se perçoit comme membre d'une société avec ses intérêts et ses secrets à partager. II découvre des amis réels et tient à explorer une indépendance sociale. Garçons et filles manifestent des intérêts différents et entrent en contact avec les structures sociales à leur manière.

\section{La représentation de la forme.}

C'est à ce stade que les personnages ont un sexe, des vêtements étudiés, des détails qui caractérisent leur comportement. Ils sont présentés dans un contexte, en situation.

\section{L'utllisation de la couleur.}

L'enfant raffine sa connaissance des couleurs et porte maintenant attention aux . nuances. II recherche la subtilité à travers les textures (roche sèche ou mouillée), 
les superpositions, mais n'est pas encore attiré par les clairs-obscurs, les plis dans les vêtements.

\section{L'organisation de l'espace.}

La ligne de sol est plus naturelle, courbée et rejoint le ciel. L'expression de I'horizon approche dans la représentation de deux plans (auto devant et maison derrière). Les tableaux sont souventes fois titrés.

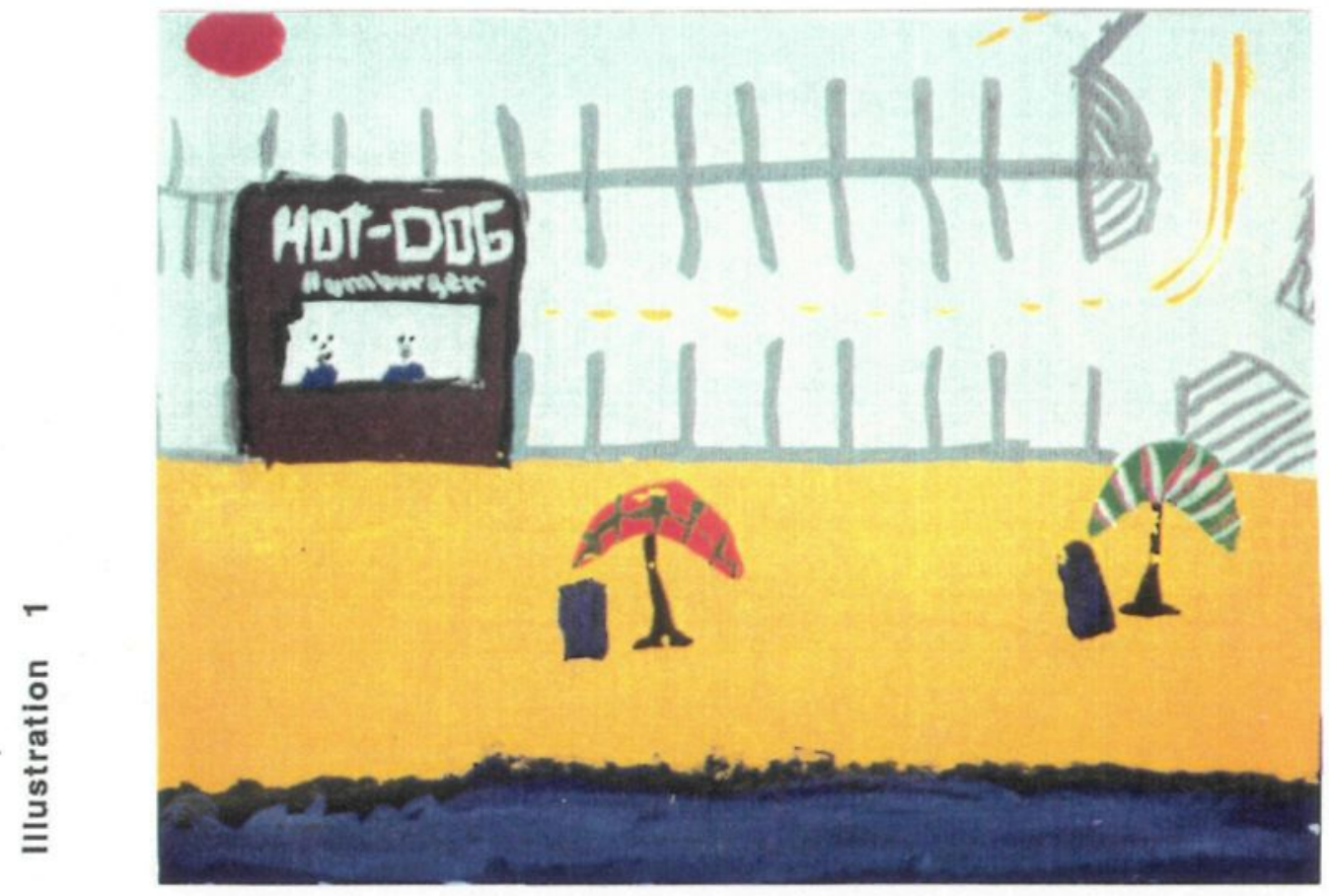

Le stade du pseudo-naturalisme ou âge du raisonnement (12-14 ans)

Cette période est importante parce que les attitudes et les habiletés qui y sont développées influenceront les réactions et les sentiments de l'adolescent face à l'art, devenu adulte. 
C'est maintenant la raison qui l'emporte, c'est une période riche en critique, c'est la fin du naturalisme et de l'activité spontanée. Les premières manifestations du stade d'expression adulte sont ainsi abordées par l'adolescent. II est de plus en plus conscient de ce qu'il voit, se soucie de ce que l'autre pense de lui, se questionne, veut savoir ce qui est important pour lui dans la vie. II ne dessine plus ce qu'il sait . des choses, mais ce qu'il voit.

Le dessin est devenu miroir dans lequel il se fait face. II se traduit surtout en bande dessinée, en caricature ou en dessin humoristique, dans des calepins, des cahiers à dessins qu'il ne présente pas à l'adulte. II se projette à travers les personnages qu'il peint, mais ne se dessine presque plus et manifeste souvent une opinion négative de son travail

\section{La représentation de la forme.}

Les articulations des membres du personnage sont représentées et l'habillement ainsi que la coiffure ont gagné en importance. Ce souci, l'adolescent le traduit par le pli du vêtement et le mouvement des tissus.

\section{L'utillsation de la couleur.}

L'enfant utilise la couleur à partir de l'expérience qu'il en a et des préférences qu'il a établies. II travaille avec des couleurs atténuées, en gradation, explore les clairs-obscurs, les ombres-lumières et veut créer une ambiance à partir de ces propriétés. II sait maintenant que la lumière peut influencer, altérer son travail. II se plait à reproduire des textures à travers les carapaces de tortues, les écailles de poissons, les crevasses dans le sol ou la rue. 


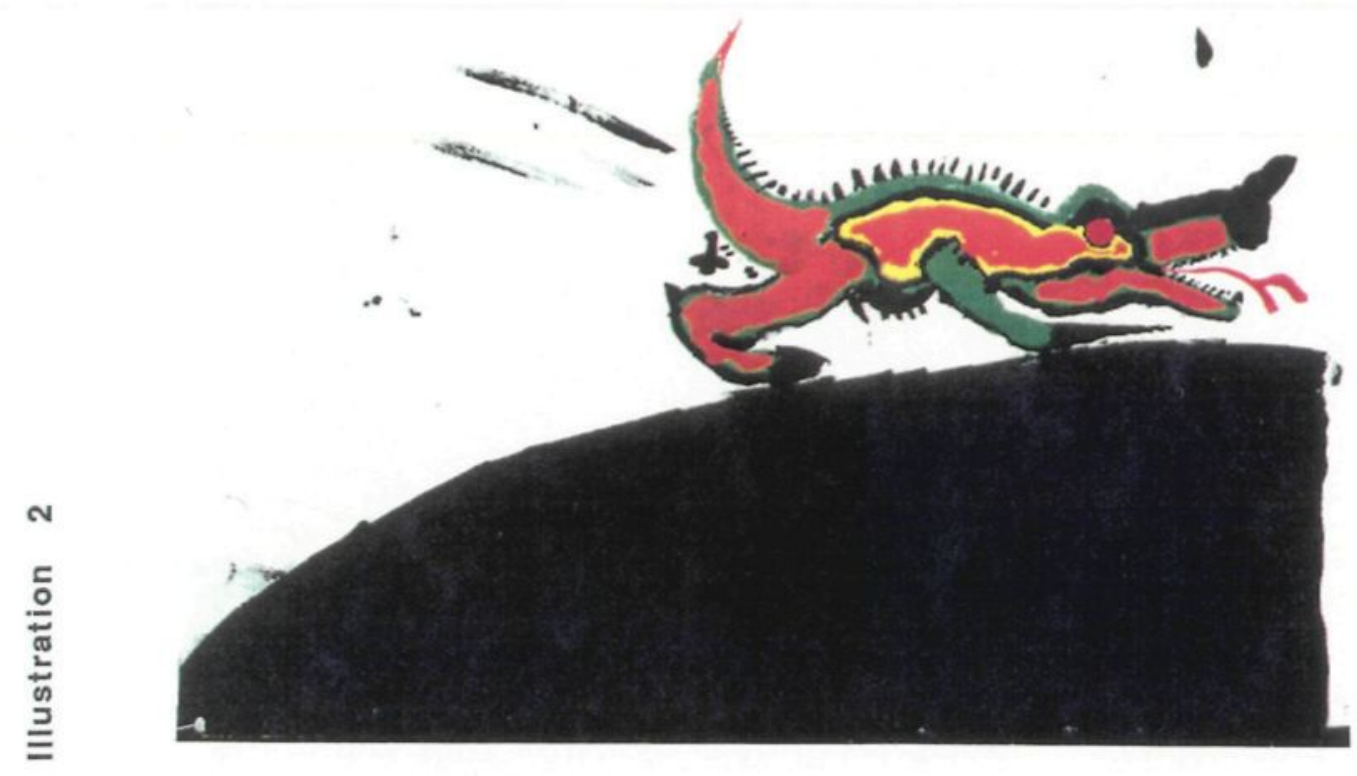

L'organisation de l'espace.

La dimension, les détails, l'intensité en couleur des objets éloignés sont réduites, l'enfant annonce ainsi l'acquisition de la notion de profondeur. La présentation d'un tableau-synthèse des stades graphiques de Lowenfeld (annexe 1) permet de situer les éléments principaux de chacun de ces stades.

Luquet (1967), pour sa part, articule sa compréhension du développement du dessin chez l'enfant autour de la notion d'acquisition ou d'appropriation du réalisme. Le dessin de l'enfant est réaliste par les sujets qu'il traite et par la nature de ses motifs. C'est un ensemble de lignes qui représente une forme et qui témoigne de l'observation en éveil. 
Le dessin trouve son importance dans l'intention, dans la manifestation de la représentation mentale beaucoup plus que dans le résultat. Luquet présente quatre stades de développement du dessin chez l'enfant: le réalisme fortuit, le réalisme manqué, le réalisme intellectuel et le réalisme visuel.

Au stade du réalisme fortult, l'enfant trace des lignes pour le plaisir de laisser une marque, puis il prend conscience qu'il possède un pouvoir créateur et recommence l'activité; son dessin devient alors intentionnel. En découvrant que les tracés veulent dire quelque chose, il crée l'analogie entre un de ses tracés et un objet réel, d'abord de façon accidentelle, puis de façon constante. Son interprétation du travail est davantage l'idée qu'il a à l'esprit et devient une représentation volontaire. A la fin de ce stade, il peut annoncer son intention, ce qu'il va faire.

Au second stade appelé réalisme manqué, l'enfant veut représenter l'objet mais se heurte à des obstacles de motricité fine, de capacité d'attention limitée et de disproportion. Lorsqu'il dessine, il ne pense qu'à ce qu'il trace et oublie les autres éléments du tableau. L'importance qu'il attribue au moment présent lui fait exagérer la dimension des objets ou la rapetisser s'il manque d'espace.

La période du réalisme intellectuel est caractérisée par la ressemblance que l'enfant veut donner au dessin. Pour cela, l'objet doit contenir tous les éléments réels, même invisibles, soit du point de vue d'où il est envisagé ou d'un autre point de vue possible. Il cherche à représenter l'usage qu'il fait de l'objet, sa dimension, l'importance qu'il a pour lui. C'est ainsi que le contour d'un champ, les racines de pommes de terre, les joues ou le ventre sont représentés. La tête et le corps du 
personnage sont des éléments différents qu'il divise par un trait au niveau du cou ou qu'il distingue par la formation de deux contours tangents. Son incapacité synthétique l'amène à détacher des détails qui, dans la réalité se confondent (chapeau posé sur le dessus de la tête, train posé sur le dessus d'une rail...). Transparence, rabattement et plan à vol d'oiseau sont des moyens graphiques fréquemment observés à ce stade.

La période du réalisme visuel voit la face et le profil se départir l'un de l'autre, la transparence se transformer en opacité et le rabattement en perspective. La narration graphique apparaît alors sous plusieurs formes: une scène ou plusieurs scènes de l'histoire sur le même dessin ou encore un mouvement décomposé dans le même dessin (grimper à un arbre).

Luquet pense que l'enfant aborde l'utilisation de la couleur de deux façons: réaliste ou décorative. Réaliste lorsque la couleur est essentielle à l'objet (plante, ciel, eau) et décorative quand l'objet pourrait être d'une autre couleur.

Stern $(1963,1966)$ a, pour sa part, bâti un modèle d'éducation artistique dans le quotidien, en vivant avec les enfants, en observant leurs comportements face à la peinture, en ajustant son action éducative petit à petit. II a davantage orienté ses énergies vers l'organisation matérielle du miliēu, la présence de l'éducateur et l'analyse de la structuration des signes, des phénomènes généraux qui composent la grammaire du langage plastique chez l'enfant. 
L'enfant peint ce qu'il a vu, il s'approprie le monde et traduit ses préocccupations; ce qu'il dessine est une projection de son corps, un miroir qui filtre à travers des apparences le visage intérieur, c'est le complément de l'expression verbale. Son tableau est l'image du moi (Stern, 1961), ses éléments forment un portrait transfiguré, un inventaire de sensations, de sentiments, de connaissances, l'ensemble de données dont la plupart sont inexprimables par un autre moyen.

Selon Stern, l'enfant adopte une attitude constructive face à ses angoisses lorsqu'il les enregistre sur papier, lorsqu'il les met à jour; c'est un peu la résolution d'un problème ou du moins la prise de conscience.

Si l'adulte peut lire l'évolution plastique de l'enfant à travers ses réalisations, cet épanouissement prouve que le travail a atteint les couches profondes de sa personnalité, que le travail fait partie des acquis plus que des impositions, et c'est dans cette perspective que Stern réfléchit. La notion d'évolution étant, selon lui, un des grands principes de l'art enfantin. II ne s'agit pas d'imposer une idée, parce que l'enfant adoptera alors la solution de facilité; il s'agit de favoriser le choix, d'exciter l'imagination, d'exercer l'esprit de décision permettant ainsi à chacun de préciser sa nature, son identité.

A partir de sa conviction de la nécessité è du besoin de peindre chez l'enfant, Stern (1967) a cherché à définir les points majeurs de la réussite de l'atelier de peinture et le rôle de l'éducateur. II a vécu en atelier avec les enfants. II les a observés, a recueilli des dossiers de peintures d'enfants, les a analysés, a cumulé la 
fréquence d'apparition de certains symboles et nous a proposé un plan détaillé de ce qu'il croit être l'architecture du langage plastique.

Ce langage (Stern, 1963) se traduit par l'image et son symbole; l'image étant la formulation plastique de sentiments et le symbole étant le réservoir, la grammaire, le langage, la communication rapportant les sensations et les connaissances de l'enfant. Selon lui, l'art enfantin naît de deux facteurs: la faculté de jouer et l'obéissance à la dictée de l'expression. Cette expression qui satisfait un besoin profond, se concrétise en un langage formel selon des mécanismes établis dans la logique un peu comme le langage verbal et ce, sur trois plans d'expression: le présent, le passé rapproché et le passé lointain. Les formes, les couleurs, l'utilisation de l'espace, la notion de temps, de mouvement sont utilisés symboliquement comme des signes de la structure de ce langage.

Stern (1966) n'envisage pas l'évolution graphique de l'enfant par l'apparition de stades graphiques, il organise plutôt ce cheminement par la formation d'une grammaire plastique. Cette grammaire se compose de la représentation des formes de base et de la représentation des images résiduelles.

\section{La représentation des formes de base.}

Stern a bâti une grammaire des formes de base à partir des premiers schémas reconnaissables fréquemment utilisés par l'enfant pour représenter sa connaissance du monde. II a, par la suite, traçé un cheminement des compositions que l'enfant expérimente, un cheminement des relations qu'il établit entre les formes. Ces nouvelles formes sont qualifiées de dérivés ou d'images résiduelles, c'est une sorte d'enri- 
chissement du vocabulaire de base, une précision visuelle qui se construit avec la pensée de l'enfant.

Au début, le geste de peindre, cette manifestation d'abord naturelle est une sorte d'expression vitale et ne représente ni tout à fait l'objet, ni tout à fait l'espace. Ce sont des spirales, des hachures, des traces incontrôlées, des traits équilibrés ou déséquilibrés: c'est la période de gribouillis qui donnera naissance au premier stade de formes arrondies.

Ce qui est arrondie est la première forme que l'enfant reconnaît, qu'il intègre, puis, avec la connaissance qu'il a de lui et de son monde, il raffine son geste, gagne en emprise sur l'outil et nous présente boucles, ovales, gouttes, ellipses, carrés estompés. Ces manifestations sont la base d'une grande structure qui se complexifie avec l'exercice, l'acquisition de connaissances et d'expériences émotionnelles.

Le cercle est donc le point de départ de la figure humaine, la goutte se perfectionne en devenant prémaison, poisson, et le carré deviendra un type de maison ou un véhicule motorisé (annexe 2: a).

Ces formes évoluent d'abord séparément puis se réunissent par juxtaposition ou interpénétration durant le processus. Elles deviennent images d'objets ou contenus (personnage, maison) et images d'espace ou contenants (annexe 2: b, e, f, g, v).

Après la période de formes arrondies, c'est l'élaboration des images-mots alignées sans lien apparent, disposées un peu partout dans l'espace feuille (banane, so- 
leil, trace de lapins ...). Ces images sont le reflet des préoccupations du moment; elles se lisent à travers un discours simple et à travers l'environnement quotidien d'un tout-petit. Ce sont le bonhomme, la maison, la fleur, l'arbre, le nuage, le soleil, l'animal, le bateau. Même si ces formes ne sont pas toujours représentées de façon reconnaissable pour l'adulte, c'est ce à quoi l'enfant pensait lorsqu'il peignait.

En énumérant les éléments placés un à côté de l'autre, l'enfant s'amuse à répéter et à transformer les formes simples; le soleil est à la fois personnage, biscuit, raisin ... L'enfant a maintenant conscience qu'il peut se servir des mêmes symboles pour représenter des éléments différents. La construction de nouvelles formes se fait alors par association et comparaison des éléments et des moyens connus avec le nouvel élément. Cette construction, Stern la qualifie d'images résiduelles de l'évolution du bonhomme. Elles constituent le répertoire d'images primaires du langage plastique, l'enrichissement, les variations de la forme de base et forment le vocabulaire dont se sert. l'enfant, ce qui explique à la fois leur permanence et leur fréquence. Elles sont la preuve que ce que dessine l'enfant est une image par procuration de lui-même. II a alors découvert une structure commune à chaque groupe d'éléments, un système.

Des images alignées un peu partout sur la feuille, surgissent ensuite les thèmes que l'enfant explore en leur donnant une dimension temporelle (jour, nuit, saison, vent ...). Il poursuit l'enrichissement de sa connaissance du monde et la traduit en langage du ressenti, à travers la représentation de concepts qui le fascinent, en nous livrant sa construction du dedans. 


\section{Évolution de la forme de base: bonhomme.}

La représentation du bonhomme grandit avec l'expérience ou la conscience du moi selon trois lignées généalogiques:

\footnotetext{
- têtard - patate - cloche

- têtard - deux membres

- têtard - autour d'une tige (annexe 2: h).
}

Durant son évolution, le bonhomme têtard à quatre membres (1ère lignée) du stade préfiguratif devient le bonhomme patate, puis le bonhomme cloche. La pieuvre, le soleil, la table rabattement, la table debout sur deux pieds, le personnage maison et la maison sont des images qui se développent conjointement avec ce type de personnage. Ce sont des images résiduelles qui se construisent à partir de cette lignée du bonhomme. Le personnage buste en est également un prolongement (annexe 2: i, j).

Le têtard-deux membres ( $2^{\circ}$ lignée) évolue en permettant de préciser la route, le nombril, le bateau à voile, la tente, la barque, la pré-maison et la maison (annexe 2: $k, 1)$. A partir du têtard-autour d'une tige ( $3^{e}$ lignée) se développe des images de fleurs et d'arbres (annexe 2: I, m, n, o). Pendant que la représentation du bonhomme se construit, les images dérivées entrent ainsi dans l'agencement du tableau enfantin et s'ajoutent au vocabulaire plastique de l'enfant. 


\section{Les Images résiduelles.}

Les images résiduelles du bonhomme sont souvent exprimées par l'enfant à travers le rabattement. Le rabattement est un moyen que l'enfant utilise pour dire ce qu'il ressent de son environnement spatial. Ce peut être un rabattement rayonnant (ex.: table) ou un rabattement axial (ex.: route). Ce moyen se compose de trois éléments: corps, centre et membre dans le cas du rabattement rayonnant, corps, axe et membres dans le cas du rabattement axial. Par cette ressource, l'enfant explore, manipule, définit l'objet qu'il veut représenter en recomposant la forme de base pour enfin lui donner une représentation synthèse (annexe 2: $m, n, 0$ ).

Ce moyen, d'abord simple et facilement identifiable dans la peinture du jeune enfant, se perfectionne en image plus subtile un peu plus tard, à travers les franges, les reflets dans l'eau ... Stern dit à ce sujet qu'il y a "entre une image évoluée et sa forme primaire, la même relation qu'entre un mot français moderne et sa forme originale latine". Les structures de base demeurent stables mais leurs images évoluent. Les images résiduelles sont l'adaptation des signes à l'expression.

Les principales images résiduelles provenant de la première lignée sont: la table, l'animal, la maison (annexe 2: i, p, q). Les images dérivant de la deuxième lignée sont: la route et la ligne axiale (annexe $2: k, n, 0$ ). La troisième lignée produit: l'arbre, la tente, le moulin et le signe $V$ (annexe 2: r, s, t, u).

On peut supposer que les images résiduelles que Stern a classifiées peuvent être plus nombreuses, mais celles qu'il a représentées demeurent les principales. 


\section{La transparence.}

La transparence est une manifestation d'abord influencée par des expériences de connaissances, des expériences d'ordre intellectuel, comparativement aux manifestations énoncées précédemment qui sont plutôt de nature émotive (annexe 2: v). L'enfant dessine maintenant ce qu'il sait de son environnement spatial.

\section{L'utilisation de la couleur.}

Stern (1976) n'a pas recensé de manifestations spécifiques au sujet de l'utilisation de la couleur par l'enfant. II pense que le jeune enfant la choisit selon la fantaisie du moment, avec l'affection qu'il lui accorde et la disponibilité ou l'accessibilité qu'elle présente. La couleur est une image donnée aux sensations, elle est expression. Le choix répété d'une couleur est la preuve d'une préoccupation qui est exprimée aussi longtemps que dure la motivation.

Stern (1959) accorde de l'importance à l'ordre de présentation des couleurs sur la table-palette. II propose une invitation à la couleur.

\section{L'organisation de l'espace.}

Au fur et à mesure que le personnage se raffine, les images résiduelles aussi prennent corps dans l'espace. L'ordre dans l'environnement devient préoccupation, la perception de la réalité augmente et se fait voir dans tous les aspects de l'agencement. Les détails et les costumes, le face et le profil, le geste et l'humour à travers l'exagération ou la déformation d'un trait; voilà autant d'éléments avec lesquels l'enfant exprime maintenant son style, son vocabulaire personnel. 
Le personnage a un nom, il joue un rôle dans le tableau et est élaboré dans un contexte. Cette évolution est parallèle à celle de l'espace. Au début, la notion d'espace se confond avec le remplissage de feuille. Au stade de rabattement, l'objet est une figure sans définition spatiale, puis lorsque cet objet bascule en position verticale, il entre en relation avec le sol. La ligne du sol et du ciel permet alors de reconnaître les éléments du tableau dans une relation plus ou moins définie dans l'espace. Lorsque l'objet présente une existence horizontale et verticale ou une existence spatiale, Stern prétend que la feuille est remplie et l'espace conquis.

Dans son ouvrage intitulé L'activité créatrice et l'enfant, Clasca Ozinga (1969) a étudié le dessin d'enfant en observant l'évolution et les lois du pouvoir figuratif de celui-ci sous deux aspects: l'évolution du griffonage vers le volume et l'évolution de la tâche vers le volume. Son travail s'inspire des auteurs cités préçédemment.

Pour elle, cette évolution se traduit par trois périodes: la période de griffonage, la période de l'idéoplastique ou de la représentation du concept et la période du naturalisme, du réalisme visuel ou du dessin de l'image observée.

La période de griffonnage est une écriture sans image, c'est un geste moteur avant tout. A l'intérieur de la période idéoplastique, il y a la phase schématique, la phase du premier sentiment de la ligne et de la forme et la phase de la silhouette ou du contour. On retrouve quatre phases dans la période du naturalisme: la phase latente ou négative, la phase chaotique ou de recherche, la phase d'ordonnancement et la phase du plus ou moins grand perfectionnement. 
Elle soumet des caractéristiques concrètes de manifestations graphiques de la période idéoplastique ou période de représentation du concept qui s'étend de la $3^{e}$ à la $11^{\circ}$ années de vie de l'enfant. Un peu dans la lignée de Lowenfeld, elle affirme qu'à cet âge, l'enfant représente ses sujets selon la valeur qu'ils ont pour lui, selon ce qu'il en sait et ce, en fonction de ses rapports avec le milieu. Le dessin est un moyen créateur qui aide à maîtriser la réalité. Elle a répertorié ces manifestations idéographiques ou procédés graphiques que l'enfant exprimè à travers sa recherche de lois du pouvoir figuratif, dans une étude menée dans plusieurs pays, dans des milieux socio-culturels différents et sur une période de 25 ans. Au nombre de 12, ces manifestations permettent de préciser les stades graphiques. Ce sont des éléments que l'on retrouve dans le cheminement de l'organisation spatiale et que l'enfant développe tout au long des stades élaborés par Lowenfeld.

\section{Exemplarité.}

Par l'exemplarité, l'enfant représente l'objet selon l'importance qu'il a pour lui et non tel qu'il est en réalité. II s'agit plutôt, pour le dessinateur, de nous dévoiler toute la connaissance qu'il a de l'objet. Cette représentation est la première manifestation de la notion de volume. Notons que c'est d'abord Luquet qui a employé ce terme pour expliquer cette caractéristique.

\section{Rabattement.}

A ce stade, l'enfant est au centre de l'expérience, il veut exprimer tout ce qu'il a vu et il le fait en s'appuyant sur une ligne ou autour d'un cercle, d'une ronde. II est en quête de notion de conservation de la matière, de réversibilité. 
Inclinaison.

Par ce procédé, l'enfant dépose ses objets et ses personnages sur une ligne qui fait état de sol et tout est vu à la perpendiculaire par rapport à cette ligne (annexe 3).

\section{Exagération de l'objet.}

La représentation de ses objets ou de ses personnages est traduite selon la place qu'il occupe en lui. La dimension de la représentation de ses formes est affective et présente donc des détails oubliés ou amplifiés selon le moment.

\section{Transparence.}

Par la transparence, l'enfant nous fait voir à travers les murs d'une maison, à travers le ventre d'une mère (annexe 3).

\section{Répétition automatique.}

Des objets, des personnages presqu'identiques seront dessinées en grand nombre, c'est là une façon de désigner la quantité et le plaisir de répéter un nouveau procédé.

\section{Juxtaposition.}

L'enfant dessine les objets les uns au déssus des autres, à côté les uns des autres ou tout simplement séparés (annexe 3). 


\section{Symétrie.}

C'est une recherche d'équilibre entre la droite et la gauche, le haut et le bas que l'enfant entreprend en plaçant ses formes répartis également sur la feuille.

\section{Perspectlve étagée.}

Par cette démarche, le dessinateur dépose sur une ligne horizontale ou ligne de sol tous ses objets ou personnages et s'il manque d'espace, il dessine une deuxième ligne afin de répondre à son besoin (annexe 3).

\section{Perspective plan.}

L'enfant représente maintenant l'image selon trois plans: l'avant-plan, le plan central et l'arrière plan (annexe 3).

\section{Perspective à vol d'olseau.}

De la juxtaposition, le dessinateur passe à cette technique qui se lit un peu comme si l'on volait au-dessus du travail.

\section{Perspectlve polygone}

Un côté de l'immeuble est dessiné en fuite tandis que l'autre n'existe pas pour l'enfant.

Au sujet de l'utilisation de la couleur, Ozinga a simplement noté que le jeune enfant utilise les couleurs criardes, puis il les harmonise et après l'âge de 11 ans, il leur accorde une nuance. 
Elle souligne également l'évolution du mouvement dans le dessin d'enfants à partir de recherches effectuées par Georges Rouma dans son livre Le langage graphique de l'enfant, où il distingue quatre phases de développement. Dans la première phase, le mouvement est absent et l'enfant dessine les formes sans rapport réciproque entre elles. Peu à peu, il représente ces formes en relation mais n'arrive pas encore à exprimer l'action. Ce n'est qu'à la troisième phase d'évolution du mouvement que l'enfant exprime sa compréhension par la représentation des personnages en action, une action présente, mais rigide et maladroite. Dans la quatrième phase, l'enfant arrive à traduire une reproduction exacte du mouvement et de l'action, il a alors 16 ans.

Ces auteurs ont souligné des approches différentes afin de répondre à leurs objectifs. Lowenfeld et Ozinga ont, pour leur part, étudié des productions enfantines provenant de contextes différents et ce, avec une perspective esthétique. Stern et Luquet ont davantage étudié les productions de mêmes enfants dans un contexte pédagogique et ce, sur une longue période.

Chacun des auteurs retenus dans la présente étude apporte des informations qui permettent de lister un nombre suffisant de traits distinctifs dans chacun des stades graphiques à travers lesquels l'enfant bâtit un langage plastique. Ces traits distinctifs seront retenus pour constituer une grille d'analyse afin d'observer dans chacune des peintures réalisées durant l'expérience s'il y a présence des caractéristiques du stade graphique propre aux pré-adolescents. 
En questionnant l'évolution des stades graphiques, l'importance de l'organisation matérielle d'un atelier de peinture retient l'attention. La recension des écrits fera maintenant valoir quelques études sur l'environnement physique afin d'en saisir l'importance.

\section{Les études sur l'importance de l'organisation matérlelle de l'espace}

Lorsqu'on parle d'environnement, on se refère à un ensemble de variables qui ont une influence sur le comportement des individus. C'est ainsi que la variable milieu socio-économique, âge, sexe, interaction avec les pairs, interaction avec les objets et aménagement de l'espace sont étudiés pour évaluer leur influence sur le développement ou les comportements des individus (Pommerleau et Malcuit, 1983). Dans la présente étude, seule la variable aménagement physique est retenue.

Une recherche qui vise la clientèle préscolaire, publiée par Goetz et Allen (1983), considère que le degré d'impact d'un programme varie selon les limites physiques d'espace, selon la quantité de matériel et d'équipement disponibles et selon l'interaction entre l'adulte et les enfants. D'après elles, la santé et la sécurité sont les standards minimum d'environnement nécessaires au bien-être de l'enfant mais les effets de l'environnement physique sur la qualité de vie de l'enfant sont aussi prioritaires. Ces priorités doivent être orientẻes vers un arrangement fonctionnel et attrayant du matériel et de l'équipement. L'accès aux espaces de rangement doit être invitant pour l'adulte afin qu'il établisse un roulement de matériel et pour l'enfant afin qu'il ait accès facilement à ce matériel. 
Kritchevsky, Prescott et Walling (1969) considèrent, pour leur part, que plus il y a d'activités offertes, plus il y a variété dans les espaces de jeux, plus l'aire est aménagée minutieusement, plus les comportements fonctionnels se manifestent. L'usage adéquat de l'espace, le choix d'un matériel présentant un éventail d'expériences sensorielles sont autant de facteurs de développement optimal. Enfin, ces auteurs croient que l'organisation physique, l'agencement de l'équipement, le mobilier, les facilités qu'offre l'architecture supportent ou découragent l'enfant dans sa démarche.

Proshansky et Wolfe ont noté qu'il y a deux effets importants de l'organisation matérielle sur le processus d'apprentissage en classe. On peut qualifier ces effets de réversibles. II s'agit, d'une part, du message symbolique qui est donné par le choix de l'organisation physique et, d'autre part, des conséquences ou comportements fonctionnels qui se manifestent face à l'arrangement du mobilier.

Nash (1981) énonce que naturellement nous organisons l'espace autour de nous selon ce que nous y faisons. II souligne que les décorateurs nous rappellent que le choix des couleurs, le choix du type d'ameublement suggèrent des comportements dans une maison. Il observe des situations toutes simples, comme l'aménagement des laboratoires de science pour les classes avancées et affirme que l'aménagement de l'espace peut être pensé dans le but de favoriser des apprentissages spécifiques. Après avoir mené une étude sur une période dè trois ans dans 19 classes témoins et 19 classes expérimentales avec des enfants de 4 et 5 ans, Nash dégage le principe suivant: la disposition du matériel qui se ressemble dans des coins voisins favorise le transfert d'apprentissage. Par contre, la séparation physique des activités donne un message contradictoire à l'enfant et nuit à son développement. 
Dans une étude sur l'influence de l'environnement physique, Phyfe-Perkins (1979) souligne que le matériel utilisé en classe doit être choisi en tenant compte de cinq critères: l'attention qu'il suscite, la valeur sociale qu'il a, la variété et la complexité qu'il propose et enfin, il doit être offert en quantité suffisante.

Deux autres recherches, visant des aménagements spécifiques cette fois, ont été recensées. II s'agit du travail de l'équipe PRAMÉ (Projet d'Analyse du Matériel Éducatif des classes maternelles) qui a étudié l'exploitation du matériel dans l'aire des jeux symboliques en maternelle et du travail de Fortier-Lavoie qui a mis en place, dans une classe de première année, une organisation matérielle en fonction de l'apprentissage de la lecture.

Les indices provenant de l'étude menée par Pramé (Thériault et al., 1987b) sur l'exploitation du matériel dans l'aire des jeux symboliques démontrent qu'il semble y avoir un lien entre la présence de critères d'organisation matérielle et celle des comportements appropriés que l'on s'attend à retrouver dans cette aire.

Cette équipe a d'abord analysé, dans sept classes maternelles, les comportements que présentaient les enfants dans cette aire, sous trois caractéristiques: l'ordre de construction ludique, le souci de vraisemblance et l'attribution des rôles complémentaires. Ces caractéristiques ont été, pàr la suite, associées à quatre critères d'organisation matérielle dans l'aire des jeux symboliques: l'agencement logique du mobilier, la diversification des scénarios suggérés, la présence d'un matériel complet pour les rôles suggérés et enfin, l'intimité de l'aire. 
Cette étude a démontré que l'agencement logique du mobilier suggère à l'enfant des thèmes et des scénarios. II suscite des comportements appropriés à la fonction de l'aire des jeux symboliques. L'absence de variété du matériel offert pour les rôles suggérés limite l'activité, empêche l'interaction entre les rôles et engendre des comportements inappropriés. L'étude dégage donc l'importance de prévoir un aménagement logique de l'aire et l'accès à un matériel complet pour les scénarios suggérés afin de permettre la réalisation et l'expression de rôles élaborés.

Selon Fortier-Lavoie (1984), enseignante en première année, la croissance se réalise au contact des objets et des personnes et la connaissance découle des instruments eux-mêmes, s'ils sont bien conçus et présentés au bon moment. Le matériel . doit être suggestif, à la portée de la main, varié et doit présenter des degrés de diffiçulté.

La diversité des situations proposées, l'exploration active d'un environnement . riche et une grande variété de matériaux manipulables deviennent ainsi des facteurs facilitant le développement.

Cette orientation poursuit la démarche amorcée au préscolaire à travers le fonctionnement en atelier; c'est ainsi que lorsque l'apprentissage de la lecture est visé, un "coin salon" et un "coin pouf", par exémple, sont aménagés dans le but de motiver l'enfant à cette activité, de lui permettre d'être participant à la démarche, de favoriser en lui un choix de lecture signifiante, d'encourager la manipulation des lettres par le jeu. 
À l'intérieur de la classe, l'aménagement du matériel est planifié de façon à créer des aires de travail. Le "coin salon" est prévu pour les lectures individuelles et collectives, les causeries et les jeux. Le "coin pouf" est prévu pour la lecture collective, le mot fichier, le théâtre de marionnettes, la présentation de productions et le travail individuel et collectif. Le "salon lecture" offre des livres ou revues de tout genre, des livres faits par les enfants, et prévoit une rotation afin de maintenir l'intérêt.

Le matériel est inventorié sous quatre rubriques: le matériel de base, le matériel de soutien, le matériel de production et le matériel de lecture. Chacun de ces types de matériel poursuit des objectifs particuliers, supportés par un contenu approprié.

Ainsi l'organisation matérielle est un outil de travail pour l'apprentissage de la lecture et l'enfant vivant dans cette démarche, devient un lecteur qui comprend ce qu'il lit, qui aime lire, qui apprend à chercher activement les informations nécessaires à son développement.

Lowenfeld, Stern et Ozinga ont égalemnt réfléchi sur l'organisation matérielle de l'atelier de peinture. Ils ont arrêté leur réflexion, avec un degré différent d'importance, sur le type d'organisation matérielie à privilégier afin d'offrir à l'enfant les conditions optimales de développement du langage plastique.

Pour Lowenfeld, l'utilisation du matériel se fait à partir de quelques données techniques, mais la découverte de ses possibilités vient dans l'ensemble de la curio- 
sité de l'enfant. II s'agit de créer une atmosphère de découverte face au matériel familier et l'utilisateur se sentant davantage impliqué, répondra alors positivement aux tâches de production, de rangement, de distribution et d'approvisionnement.

II prétend que la peinture, réalisée sur une table ou sur le sol, favorise l'apparition de la transparence à cause de l'approche dans toutes les directions qu'elle propose puisque l'enfant peut circuler, se déplacer autour de la feuille. II ne préconise donc pas d'organisation spécifique, il mise sur une organisation spontanée.

Lowenfeld considère donc l'accessibilité et le choix du matériel mais sans modèle précis d'organisation. II s'agit d'offrir un matériel simple, contrastant et varié, en tenant compte des limites de l'enfant.

However, there is much variations among children, and even the material that the child uses influences how he draws (Creative and menthel growth, 1970, p.119).

Pour Stern (1970), l'acte de peindre est avant tout une confidence, un secret que livre l'enfant. S'exprimer, c'est raffiner un besoin naturel par le renforcement de moyens techniques. L'enfant qui peint régulièrement devient observateur, il devient plus sensible et attentif à ce qui est autour de lui. Pour cela, il doit produire ses réalisations picturales dans des conditionș particulières. C'est ainsi que Stern affirme que: organisation, discipline, rigueur matérielle, ordre, simplicité efficace et transparence sont les principes du travail éducatif.

Éduquer, c'est favoriser l'éclosion des valeurs personnelles. Lorsqu'il peint, l'enfant concrétise ses émotions, fait des expériences, entre en contact avec le monde. Ces facultés natu- 
relles peuvent devenir des moyens éducatifs si l'on crée des conditions appropriées (Aspects et techniques de la peinture, 1959, p.5).

L'atelier est toujours pareil à lui-même, l'enfant sait à l'avance ce qu'il y trouvera, ce qu'il y fera (Entre éducateurs, 1967, p.22).

Chez Stern, latelier est un petit sanctuaire; les fenêtres bloquées afin d'éviter les influences que le jour peut offrir, les murs qui invitent l'artiste, la table-palette au coeur du spectacle, les huit, dix ou 18 couleurs toujours disposées au même rang sur la table offrent une référence visuelle sécure et font acte de compagnonage. Les pinceaux de qualité obéissent a la main avec grand plaisir. Ils appartiennent à une couleur et sont utilisés selon une procédure déterminée, au même titre que les mélanges, tout est prévu pour capter l'importance du geste.

Tout est organisé pour que l'enfant sache que son travail est sérieux, qu'il mérite attention et respect. Les séances sont planifiées à l'avance (remplissage de godets, nettoyage de pinceaux, dossiers...), l'ordre, la sécurité, l'assurance de retrouver des références sont rigoureusement respectés. Chacun est ainsi assuré qu'il retrouvera couvre-tout, peinture, pinceau au même endroit, il n'a pas à questionner l'organisation matérielle, il n'a qu'à s'exprimer.

Les peintures sont soigneusement conservées dans un dossier laissant ainsi à l'enfant l'option de retour, d'objectivation et de réflexion tout en préservant l'oeuvre des critiques extérieures. 
Tout près de la table-palette, les accessoires sont disponibles (punaises, guenilles, couteaux, seringues, éventails, escabeaux ...), favorisant le contact optimal et méthodique entre le support et la peinture. La recherche que Stern a poursuivi accorde donc une grande importance à l'organisation matérielle. En effet, l'acquisition des stades graphiques est ici reliée à la facilité, à la disponibilité que l'aménagement physique offre à l'enfant.

Ozinga (1969) mentionne à travers son étude, l'importance d'un matériel varié mais ne propose pas de modèle d'organisation.

À partir des observations et des références offertes par les auteurs étudiés, une lecture des productions de peinture d'enfants devient un exercice nécessaire. Ces auteurs ont apporté des éléments susceptibles de décoder l'image que nous offre l'enfant pour situer sa réalisation à travers l'échelle des stades graphiques. Si les mêmes éléments d'évolution graphique se retrouvent à travers les modèles de Lowenfeld, Stern, Luquet et Ozinga et si le répertoire d'études concernant ce sujet est limité, il est donc justifiable d'entreprendre la présente recherche afin d'observer, d'une part, la présence des caractéristiques des stades graphiques dans les productions d'enfants de 11-12 ans et, d'autre part, d'observer si l'organisation matérielle d'un atelier de peinture en classe a une influence sur le développement du langage graphique de l'enfant.

La présente étude tentera donc de décrire l'organisation matérielle d'un atelier de peinture dans une classe d'enfants de sixième année ayant accès régulièrement à cet atelier et de répondre à la question suivante: 
Est-II possible d'observer, dans les productions des enfants de cette classe, les caractéristiques du stade graphique propre aux pré-adolescents? 
CHAPITRE II

Méthodologie 
Le deuxième chapitre de ce mémoire explique les orientations méthodologiques utilisées dans la présente étude. II s'agit de la description des sujets, de la description de l'organisation matérielle de l'atelier de peinture dans la classe, de la procédure utilisée et de la présentation du plan d'analyse des données choisi afin de répondre à la question posée.

\section{LES SUJETS}

Cette étude exploratoire se propose de décrire ce que traduisent les tableaux des enfants relativement à la présence des caractéristiques des stades graphiques des pré-adolescents. Les sujets sont un groupe d'élèves de 11-12 ans d'une classe de sixième année, soit 12 filles et 14 garçons d'une Commission scolaire de la région 02. Ils ont réalisé 399 peintures entre le 15 novembre 1987 et le 15 mai 1988. Ces productions constituent le matériel d'analyse de ce travail. Le nombre de productions réalisées par chacun des participants est listé en annexe 6.

\section{L'ORganisation matérielle de l'ATELIER DE PEINTURE}

L'aménagement physique de l'atelier de peinture a été pensé à partir du modèle table palette d'Arno Stern (1959). Dans un espace restreint, il s'agissait de réunir tout le matériel nécessaire à la réalisation d'une peinture, en minimisant les difficultés d'installation inhérentes aux réalités de fonctionnement d'une classe. 
Des panneaux couvrant une surface de huit pieds en hauteur et 16 pieds en largeur ont été fixés au mur arrière de la classe, permettant simultanément à huit enfants l'accès à cette activité pour une période d'une heure par semaine chacun.

Une table palette de 26 pouces de haut, sept pieds de long et deux pieds de large, sur roulettes, a été rangée le long du mur de travail et éloignée de ce mur, en parallèle de quelques pieds, au moment de l'utilisation. Cette table comprend:

\section{a) Sur la tablette supérleure}

- Dix godets de gouache professionnelle disposés en une rangée dans l'ordre suivant: noir d'ivoire, terre de Sienne brûlée, rouge vermillon, ocre jaune, jaune chrome clair, vert anglais fonçé, bleu outremer fonçé, terre d'ombre brûlée, jaune de chrome orange, blanc permanent.

- Dix godets d'eau disposés en une rangée parallèle aux godets de gouache et correspondant respectivement à chacune des couleurs ci-haut mentionnées.

- Quatre espaces godets de gouache additionnels utilisables au besoin en allongeant la table par les extrémités (deux espaces à chaque extrémité) [Annexe 4].

- Quatre espaces godets d'eau également additionnels accompagnant les godets de gouache.

- Trois pinceaux ronds en poils d'écureuil de un demi pouce de long et sept pinceaux ronds en poils d'écureuil de trois-quart de pouce de long déposés à l'horizontal sur un support. Chaque godet de gouache est ainsi outillé 
d'un pinceau (un demi pouce ou trois-quart de pouce) de façon permanente.

\section{b) Sur la deuxième tablette}

- Des feuilles cartridge $(18 \times 24)$.

- Des punaises servant à fixer les feuilles.

- Des couteaux servant à arrêter les coulisses de peinture.

- Des seringues sans aiguille servant à récupérer la peinture décantée dans les godets d'eau.

- Un rouleau de pellicule cellophane servant à couvrir les godets après la séance.

- Un éventail servant à sécher plus rapidement la peinture appliquée.

- Un timbre-dateur servant à indiquer le moment de production.

- Des guenilles et des éponges.

c) Sur la tablette inférieure

- Deux baguettes de 30 pouces de long aimantées, servant à ramasser les punaises tombées sur le plancher.

- Huit tabliers de tissus différents.

- Des pots de gouache en réserve correspondant aux couleurs déjà offertes.

- Des pinceaux en réserve qui accompagneront les nouveaux mélanges de couleurs sont disposés dans les espaces additionnels.

- Des abaisse-langue servant à mélanger ou transvider la peinture. 
Un berceau classeur comprenant le dossier identifié de chacun des enfants, a été placé le long du mur à la suite des panneaux. Les enfants y rangent leurs productions lorsque terminées.

\section{LA PROCÉdURE}

II s'agissait de présenter le matériel de base aux futurs utilisateurs en expliquant les modalités de fonctionnement exigées et la technique d'utilisation de ce matériel.

Les modalités de fonctionnement ont été définies à partir des informations recueillies dans les études citées précédemment, pour ensuite être adaptées aux objectifs de la présente étude.

\section{Les modalites de fonctionnement}

Trois périodes d'une heure seront bloquées durant la semaine et pourront être utilisées à cet atelier. Huit participants peuvent utiliser le matériel simultanément. Aucun espace n'est désigné à l'avance, chacun choisit son espace de travail. Chaque enfant bénéficie d'un espace fichier dans le berceau afin de conserver ses réalisations. Chaque utilisateur doit participer au rangement et au nettoyage du matériel à la fin de la séance, même si une équipe est responsable de superviser cette tâche. Toutes les productions seront conservées dans le berceau classeur. 
Les éléments considérés déterminants dans la manipulation de ce matériel ont été répertoriés, puis sélectionnés, pour ensuite être présentés aux utilisateurs sous forme de code.

\section{La technique et l'utilisation du matériel}

Les feuilles sont fixées au panneau avec des punaises et lorsqu'un utilisateur désire travailler sur une grande surface, plusieurs feuilles sont fixées suivant ce modèle: celles du bas en premier, puis celle du haut par-dessus en chevauchant de trois-quart de pouce. La table palette est plaçée à environ quatre pieds du panneau en parallèle, les godets peinture près du mur.

La liberté dans le choix du tablier, du thème, du format, des couleurs, du rythme d'exécution, de la position de la feuille, est laissée à l'utilisateur. Un pinceau est déposé à côté de chaque godet de couleur; il est assigné à ce godet et ne peut changer de couleur. Chaque couleur a ainsi son pinceau.

Le travail doit être exécuté debout. Il est important de se placer face à la couleur avant d'y tremper le pinceau. La pointe du pinceau seulement doit être trempée en premier dans l'eau, puis dans la gouache au milieu du godet. La pointe du pinceau doit être utilisée en perpendiculaire, comme unè caresse à la feuille. La peinture ne doit être appliquée que sur une surface sèche. Pour superposer une couleur, la première application doit être sèche. 


\section{Le plan d'analyse des données}

Afin d'étudier les tableaux que les enfants avaient peints tout au long de l'année, sept grilles d'analyse ont été réalisées. Ces grilles s'inspirent d'une étude effectuée par l'équipe de recherche Pramé (Thériault et al., 1987d). L'équipe a analysé des dessins d'enfants de 5 ans à l'aide de grilles bâties à partir du recensement d'éléments susceptibles d'être observés dans la production des enfants de cet âge. Les éléments retenus correspondaient à la définition du stade pré-schématique et schématique que Lowenfeld met de l'avant. II s'agissait de poursuivre l'élaboration de ces grilles par l'addition des éléments correspondants aux stades graphiques ultérieurs, soit le stade du réalisme visuel et le stade du pseudo-naturalisme, pour ensuite observer le nombre de présence et le processus de représentation de ces éléments à partir des informations livrées par les auteurs cités précédemment.

Cinq grilles de représentation de la forme, une grille d'utilisation de la couleur et une grille d'organisation de l'espace feuille ont donc servi à répertorier les éléments observables dans la production artistique des enfants de 11-12 ans (voir annexe 5).

Les grilles personnage, animal, solell, nuage, maison, arbre et arc-enciel ont été retenues parce qu'elles représentent les principales formes de base explorées par l'enfant dès son entrée dans le monde de la représentation graphique. Ces formes, tel qu'énoncées précédemment, se raffinent et se complexifient. Le présence d'éléments observables dans là production s'associe à la description d'un stade d'évo- 
lution du langage plastique et témoigne du processus d'acquisition du vocabulaire plastique.

Dans le cas du personnage, trois cases l'identifient d'abord, provenant de la première, de la deuxième ou de la troisième lignée selon l'étude de Stern (annexe 2: h). Puis, s'il se rapproche de la réalité ou est dessiné en profil, deux cases le soulignent à l'élément figuration humaine ou profil. Les éléments sont ensuite listés de la tête au pied, afin de faciliter la lecture des productions. La présence du mouvement et du costume figure en fin de grille.

L'élément costume de rôle regroupe des personnages habillés en joueur de hockey, en clown, en pirate, ... L'élément coiffure retient tous les types de chapeaux. Sous la rubrique accesssoire, on doit considérer le sac à main, les lunettes, les boucles d'oreilles, les barettes, les boutons sur le vêtement, l'imprimé dans un tissu, les souliers.

La façon dont la peinture est appliquée pour la réalisation du personnage est notée par les éléments "contour" qui informent: soit de la présence unique du contour de la forme par un trait de pinceau, soit de la présence d'un intérieur de forme différent du contour, soit d'un intérieur et d'un contour identique ou soit de l'absence du contour.

La case "autres" recense les informations supplémentaires comme la présence des dents, des pupilles, des pipes, des cravates, des moustaches, de la barbe et de cercles roses pour signifier les joues... 
'Les éléments retenus dans la grille de l'animal sont d'abord la représentation réelle ou irréelle de cette forme. Ils sont ensuite présentés de la tête à la queue. La représentation du type de peau et la position de l'animal sont notés. Enfin, le choix des couleurs utilisées est observé en regard de l'aspect réel ou irréel de l'animal représenté. La rubrique "autres" relève les informations supplémentaires.

Les grilles du soleil, de la maison et de l'arbre présentent les éléments dans l'ordre d'évolution de ces formes. La grille du nuage retient la façon dont les enfants réalisent cette forme et l'aspect réel ou irréel dégagé par la couleur du nuage. La grille de l'arc en clel est observé à travers l'utilisation de la couleur sous six aspects.

La grille, utilisation de la couleur, dresse d'abord une liste du choix de couleurs offert aux enfants dans le même ordre de présentation que sur la table-palette, puis comptabilise les mélanges réalisés.

Cette grille permet également de vérifier la façon dont l'enfant applique la couleur par le biais des rubriques contour, contour différent du contenu, contour égal au contenu, pas de contour et par la représentation réelle ou imaginaire que l'enfant veut exprimer de l'objet qu'il dessine.

Le troisième volet de cette grille identifie l'utilisation de la couleur en superposé, en nuancé, en ombre et lumière et avec texture ou motif. 
La grille, organisation de l'espace feuille, présente la série de procédés graphiques récupérés par l'enfant, dans le but d'organiser son travail dans l'espace feuille et ainsi projeter un message signifiant. Ces procédés sont présentés dans l'ordre de correspondance à chacun des stades d'après les informations théoriques mentionnées précédemment. C'est ainsi que les éléments dessinés à la verticale ou en suspension dans l'espace feuille, les formes close-contenant font partie du stade pré-schématique et sont définies dans les paragraphes qui suivent.

\section{Éléments à la verticale.}

Les formes sont disposées debout dans l'espace feuille, les unes à côté des autres, en flottement vertical et témoignent de la compréhension de la notion de haut et de bas.

\section{Éléments en suspension.}

Les formes sont disposées dans l'espace feuille, les unes à côté des autres, sans organisation précise, sans relation entre elles, et elles sont suspendus dans l'espace, en flottement.

\section{Forme close-contenant.}

Le schéma de base évolue par juxtaposition ou interpénétration présentant ainsi des images d'objets (contenu) et des images d'espace (contenant).

Le stade schématique est ponctué de plusieurs représentations, certaines sont déjà définies dans le cadre conceptuel (exagération de l'objet, inclinaison, exemplarité, répétition automatique, transparence, rabattement, perspective étagée, diffé- 
rentes séquences au même endroit, différentes actions en temps différents [pp.36, $37,38])$, les autres le sont dans les paragraphes subséquents.

Allgnements des éléments.

Les éléments sont alignés sur une ligne fictive, ils ne flottent plus dans l'espace et semblent plutôt s'organiser à l'intérieur de la feuille.

\section{Alignements bas de feuille.}

Les éléments sont représentés sur le bas de la feuille remplaçant ainsi la ligne du sol.

\section{Ligne du ciel.}

Une ligne est tracée dans le haut de la feuille afin de représenter le ciel.

Ligne du sol droite.

Une ligne est tracée dans le bas de la feuille afin de signifier la présence d'un plancher sur lequel sont déposés les éléments.

\section{Ligne du sol courbe.}

Elle témoigne d'une compréhension plus réaliste de la notion de sol soutenant les éléments.

\section{Espace sol et ciel.}

Une ligne est tracée dans le haut et une autre dans le bas de la feuille, créant ainsi un espace sol et ciel. 


\section{Ordre des éléments.}

Les éléments sont placés en ordre et ne flottent plus dans toutes les directions.

\section{Remplissage entier.}

Une couche de peinture couvre toute la feuille et le thème est ensuite travaillé sur cette couche de fond.

\section{Encadrement.}

Un cadre entoure le travail et délimite le contour de la feuille.

Les représentations du stade réalisme visuel et du stade pseudo-naturaliste sont définies dans les lignes qui suivent.

\section{Ligne du sol qui rejoint ciel}

La ligne du sol est plus naturelle, courbée et rejoint le ciel.

\section{Juxtaposition}

L'enfant dessine les objets les uns au-dessus des autres, à côté les uns des autres ou tout simplement séparés (voir annexe 3).

\section{Symétrie}

L'équilibre est recherché entre la droite et la gauche, le haut et le bas et se traduit par une répartition égale des formes sur la feuille. 


\section{Perspective plan}

L'image est représentée selon trois plans: l'avant-plan, le plan central et l'arrière-plan (voir annexe 3 ).

\section{Perspective à vol d'olseau}

Le travail est vu comme si l'on volait au-dessus.

\section{Perspective polygone}

Un côté seulement d'un immeuble est dessiné en fuite, tandis que l'autre ne l'est pas.

\section{Présence d'objets éloignés réduits}

La dimension, les détails, l'intensité en couleur des objets éloignés sont réduites, c'est l'annonce de l'acquisition de la notion de profondeur.

Chacune des peintures a donc été regardée à partir des sept grilles et lorsqu'un élément était perçu, il était coché. Par la suite, une compilation du nombre d'apparition des caractéristiques sera réalisée. Ces données numériques permettront de vérifier où en est ce groupe d'enfants par rapport aux caractéristiques des stades graphiques propres aux enfants de cet âge. 
CHAPITRE III

Description et discussion des résultats 
Le troisième chapitre de ce mémoire présente les résultats relativement aux productions des tableaux de 26 élèves de $6^{\bullet}$ année, dans un atelier de peinture faisant partie de la classe. Il s'agissait d'observer, dans quelle mesure les tableaux traduisent les caractéristiques des stades graphiques, telles qu'énoncées dans la question de recherche au chapitre premier soit:

\section{Est-II possible d'observer, dans les productions des enfants de cette classe, les caractéristiques du stade graphique propre aux pré-adolescents?}

L'analyse des résultats porte sur l'apparition des caractéristiques des stades graphiques des pré-adolescents. Des observations ont aussi été notées durant l'expérience et se veulent un effort d'explication sur l'importance d'une organisation matérielle spécifique pour cet atelier et de son accès par les élèves. Ces observations seront utilisées dans la discussion.

\section{LES RÉSULTATS RELATIFS À L'APPARITION des Caractéristiques des stades graphiques DES PRÉ-ADOLESCENTS}

L'ordre de présentation des résultats sera le suivant: les résultats relatifs à la présence de la forme personnage et des autres formes utilisées par les enfants, soit l'animal, le soleil, le nuage, l'arc-en-ciel, la maison et l'arbre, puis les résultats 
concernant l'utilisation de la couleur et enfin, ceux s'associant à l'organisation de l'espace-feuille. Des statistiques descriptives sont utilisées pour leur présentation.

\section{Les résultats de la représentation du personnage}

Les résultats obtenus pour la représentation du personnage indiquent que 17 des 26 participants ont dessiné le personnage durant l'expérience. 46 productions représentaient cette forme sur un total de 399 peintures, soit $11,5 \%$, et seulement trois des productions présentaient le personnage de profil. II semble donc que les pré-adolescents ne sont pas particulièrement portés à représenter le personnage dans leurs tableaux.

Si l'on observe plus précisément les traits distinctifs qui caractérisent chacun des stades, on s'aperçoit que les enfants qui ont représenté la forme humaine, ont davantage utilisé des traits qui se retrouvent aux stades pré-schématique et schématique que des traits spécifiques aux stades de réalisme visuel et de pseudo-naturalisme.

En effet, 202 éléments identifiant le stade pré-schématique ont été relevés à partir des sept traits distinctifs couvrant cette période. 181 éléments du stade schématique ont été cochés à partir des 12 traits distinctifs propres à cette période. Au stade de réalisme visuel, 73 éléments ont été observés à partir des sept traits distinctifs listés. 75 traits traduisant le stade pseudo-naturaliste ont été relevés. La figure 1 illustre cette situation. 


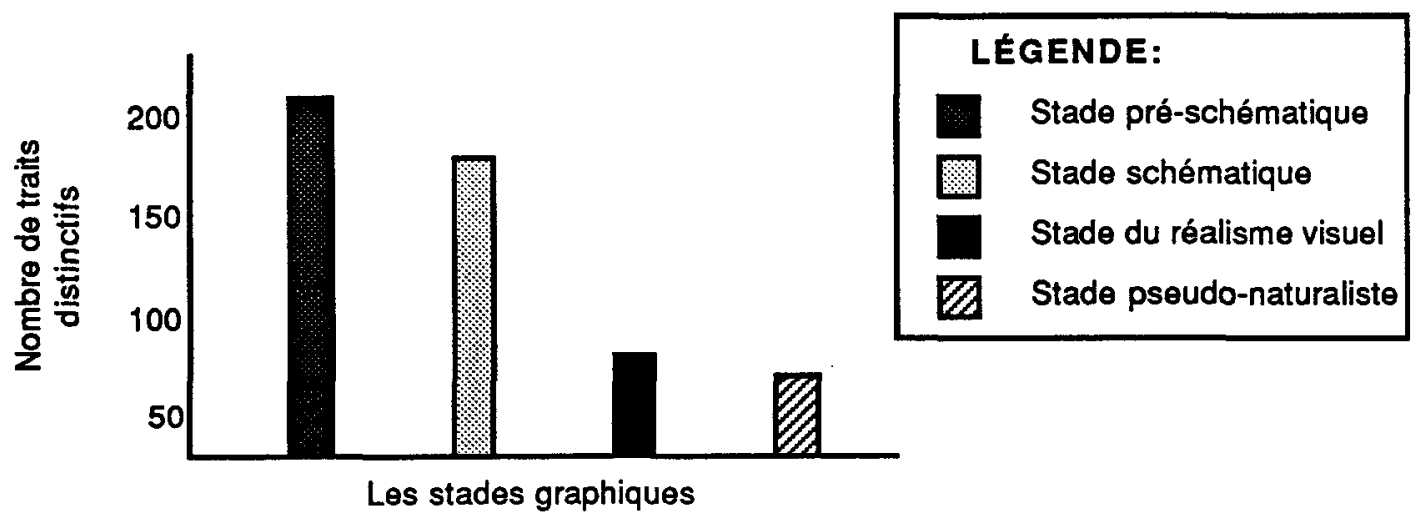

Figure 1: Nombre de traits distinctifs de la représentation du personnage pour les stades étudiés.

Une lecture plus fine de la présence des divers traits distinctifs de chacun de ces stades révèle au tableau 1 que le stade pré-schématique est bien acquis pour ce groupe d'enfants. En effet, à partir des 46 tableaux présentant le personnage, on note que les principaux éléments propres à ce stade sont présents dans une proportion significative.

TABLEAU 1

Nombre de traits distinctifs du stade pré-schématique présents dans les personnages des enfants de 11-12 ans

\begin{tabular}{|c|c|c|c|c|c|c|c|}
\hline Tralts & Tête & Yeux & Bouche & $\begin{array}{c}\text { Présence } \\
\text { cheveux }\end{array}$ & Corps & Bras & Jambe \\
\hline Nombre & 40 & 39 & 33 & 25 & 27 & 22 & 16 \\
\hline
\end{tabular}

La lecture du tableau 2 montre également que les enfants utilisent aisément les traits distinctifs associés au stade schématique. 
TABLEAU 2

Nombre de traits distinctifs du stade schématique présents dans les personnages des enfants de 11-12 ans

\begin{tabular}{|c|c|c|c|c|c|c|}
\hline Tralts & Nez & Oreilles & Sourcils & Cils & Cou & Épaules \\
\hline Nombre & 30 & 10 & 16 & 2 & 24 & 23 \\
\hline Traits & $\begin{array}{c}\text { Épaisseur } \\
\text { bras }\end{array}$ & Mains & Doigts & $\begin{array}{c}\text { Épaisseur } \\
\text { jambes }\end{array}$ & Pieds & Orteils \\
\hline Nombre & 21 & 15 & 11 & 16 & 13 & 0 \\
\hline
\end{tabular}

Si l'on regarde maintenant comment les enfants se situent par rapport aux caractéristiques des stades propres à leur âge, soit les stades du réalisme visuel et du pseudo-naturalisme, les résultats démontrent une présence intéressante des traits "expression du visage" et "longueur des cheveux", de même qu'une présence importante de la dimension "costume" et "accessoire" reliée au stade de réalisme visuel.
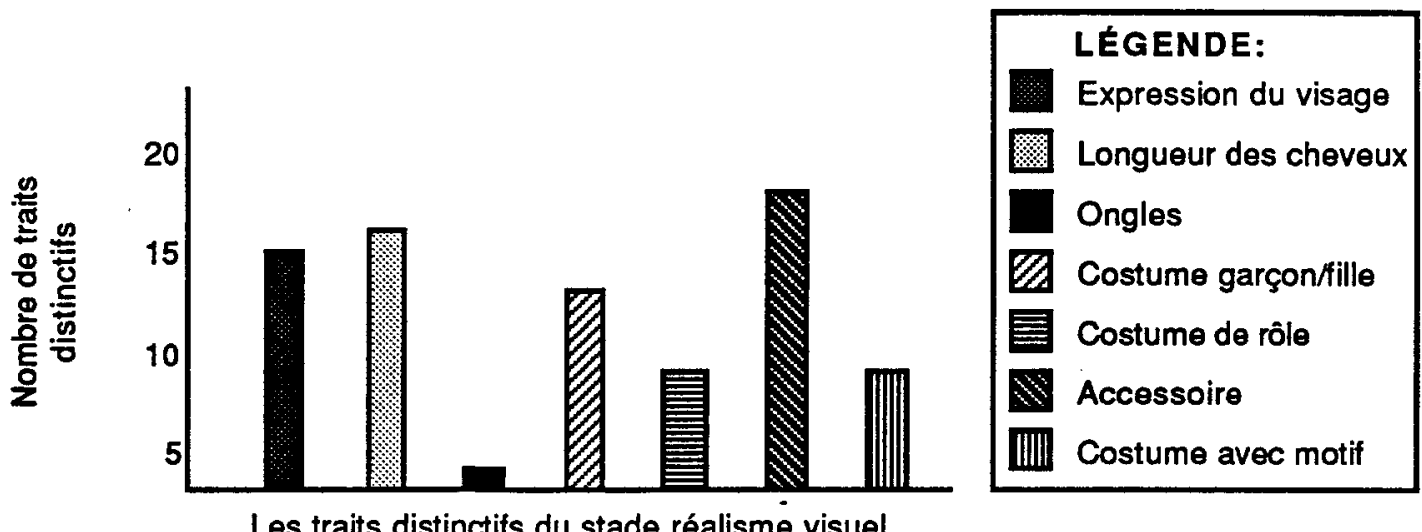

Les traits distinctifs du stade réalisme visuel

Figure 2: Nombre de traits distinctiłs de la représentation du personnage propres au stade de réalisme visuel. 
Par contre, la lecture de la figure 3 révèle que ces enfants se situent au tout début des stades pseudo-naturaliste. Les trois seuls traits distinctifs qui ont été représentés de façon signifiante se rapportent aux cheveux et s'inscrivent dans une continuité de la manifestation observée au stade précédent.

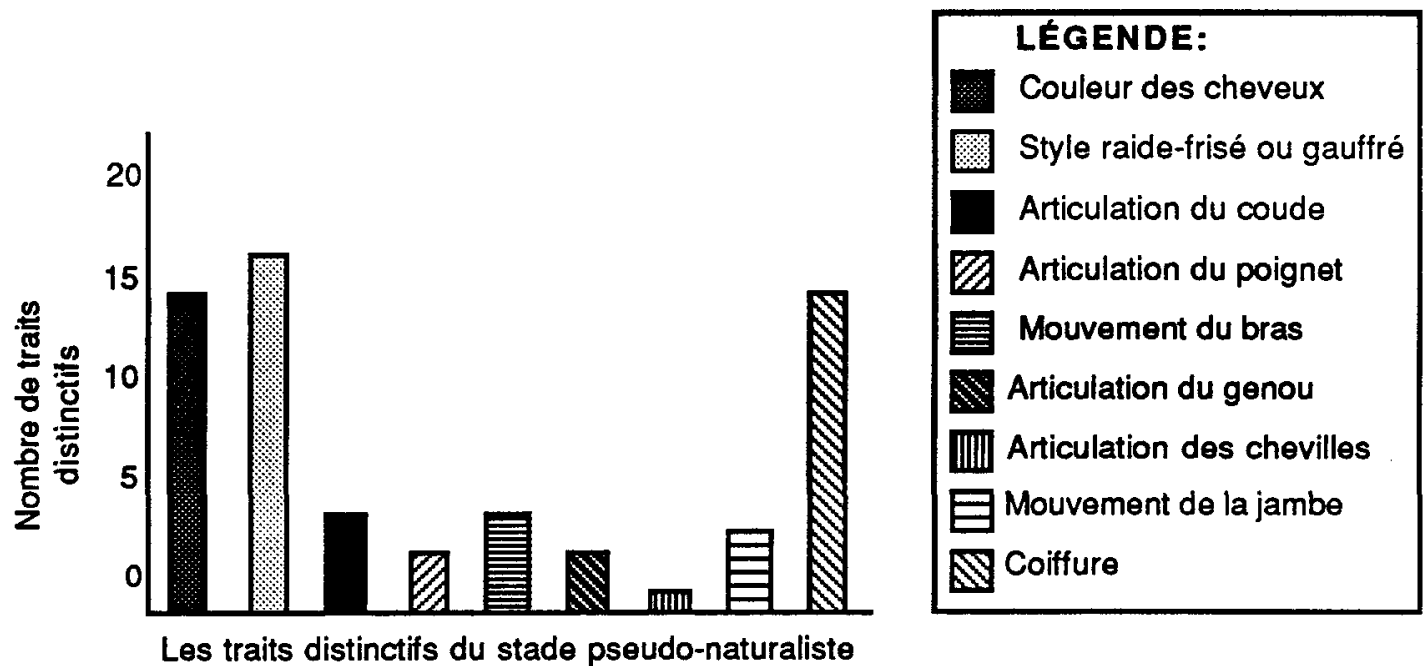

Figure 3: Nombre de traits distinctifs de la représentation du personnage propres au stade pseudo-naturaliste.

Le tableau 3 montre que les enfants utilisent les deux traits distinctifs caractéristiques des stades réalisme visuel et pseudo-naturalisme pour tracer la forme humaine. En effet, ils ont dessiné majoritairement $(65,9 \%)$ le personnage sans contour et avec contour seul dans le cas de la caricature. Les traits distinctifs associés au stade précédent sont donc peu utilisés. - 


\section{TABLEAU 3}

Nombre de traits distinctlfs relatifs à la manlère de réallser le personnage.

\begin{tabular}{lllll}
\hline Contour seul $=19$ & Contour $\neq$ contenu & $=12$ \\
Contour=contenu $=$ & 4 & Pas de contour & $=22$
\end{tabular}

Certains traits distinctifs du stade réalisme visuel et pseudo-naturalisme ont été relevés sous l'étiquette "autres". II s'agit du chapeau melon, de la tuque, du béret, des dents, des pupilles des yeux, des cercles roses signifiant les joues, de la pipe, de la barbe, de la moustache, des narines, des barettes aux cheveux, des bijoux, des cravates, des boutons aux chemises, des appliqués sur chandails et des bas de fantaisie. Le nombre de ces manifestations témoigne de l'évolution des enfants vers la ressemblance du personnage dessiné, à travers la précision qui lui est apportée.

$65,3 \%$ des enfants ont donc abordé le thème du personnage mais seulement quelques-uns d'entre-eux ont poursuivi dans cette voie. Les traits distinctifs dominants qui ont été relevés se retrouvent à des stades plus jeunes que ceux auxquels on devrait s'attendre et les traits distinctifs des stades propres aux enfants de cet âge ne sont exprimés que timidement.

\section{Les résultats de la représentation de l'animal}

Les résultats relatifs à la représentation de l'animal indiquent que 14 des 26 enfants ont réalisé 45 peintures sur lesquelles apparaissaient cette forme. Ce groupe de pré-adolescents accorde une importance équivalente à la représentation du 
personnage (46) et de l'animal (45). Les enfants ont traité ce sujet de façon réaliste à 13 reprises, tandis que 32 tableaux présentaient l'animal fantaisiste, irréel, l'animal toutou ou l'animal de bande dessinée.

La variété et le nombre de traits distinctifs utilisés dans le dessin de l'animal montrent que toutes les caractéristiques se rapportant au stade pré-schématique sont présentes et ce, dans des proportions voisines. Le tableau 4 démontre que les enfants réalisent un animal complet selon les traits observables à ce stade.

TABLEAU 4

Variété et nombre de tralts distinctifs présents dans la représentation de l'animal chez des enfants de 11-12 ans

\begin{tabular}{|c|c|c|c|c|c|}
\hline Tralts & Tête & Youx & Gueule & Corps & Pattes \\
\hline Nombre & 43 & 39 & 36 & 40 & 32 \\
\hline
\end{tabular}

Les enfants utilisent également en nombre important les traits distinctifs associables au stade schématique, comme le présente le tableau 5. Ils ont représenté plusieurs espèces d'animaux et davantage dans la position de face, ce qui permet d'observer un nombre plus restreint de l'élément queue.

TABLEAU 5

Variété et nombre de traits distinctifs du stade schématique présents dans la représentatlon de l'animal chez des enfants de 11-12 ans.

\begin{tabular}{|c|c|c|c|}
\hline Traits & Museau & Cou & Queue \\
\hline Nombre & 28 & 32 & 17 \\
\hline
\end{tabular}


Si l'on regarde maintenant comment les enfants se situent par rapport aux traits distinctifs retenus dans le stade de réalisme visuel et dans le stade pseudonaturaliste pour le dessin de l'animal, les résultats des tableaux 6 et 7 en montrent une faible utilisation. En effet, il semble que les enfants n'expriment qu'avec réserve les traits caractéristiques de ces stades. Le nombre restreint de traits distinctifs observés montre qu'ils sont à la conquête de précision et de raffinement. Ils se situent donc vers le début de l'acquisition de ces stades.

\section{TABLEAU 6}

Variété et nombre de tralts distinctlfs du stade réalisme visuel présents dans la représentation de l'animal chez des enfants de 11-12 ans.

\begin{tabular}{|c|c|c|c|}
\hline Traits & Griffes & Dents & Langue \\
\hline Nombre & 12 & 2 & 5 \\
\hline
\end{tabular}

TABLEAU 7

Variété et nombre de traits distinctifs du stade pseudo-naturaliste présents dans la représentation de l'animal chez des enfants de 11-12 ans.

\begin{tabular}{|c|c|c|c|}
\hline Traits & Poils & Plumes & Écailies \\
\hline Nombre & 11 & 4 & 6 \\
\hline
\end{tabular}

Afin de comprendre davantage la représentation de cette forme, l'utilisation que les enfants ont fait de la couleur ainsi que la position dans laquelle ils ont représenté l'animal, ont également été observées. 
La lecture des résultats de la position de l'animal fait voir une proportion importante de quadrupèdes, soit $\mathbf{4 4 , 4 \%}$. Les autres positions observées sont diversifiées, il s'agit de l'oiseau en vol, de la grenouille assise, de la panthère en plongée ... Ces représentations, souvent originales, permettent de croire que les enfants développent le souci du mouvement à travers cette forme, trait asssociable au stade qui leur est attribué, soit le stade pseudo-naturaliste.

La façon dont les enfants ont utilisé la couleur s'associe au choix de l'animal réel (13) ou irréel (32) représenté. En effet, 29 animaux ont été peints avec un choix de couleurs les rendant fantaisistes et irréels, tandis que 13 sont dessinés avec un choix de couleurs les rendant naturels.

Les résultats de la représentation du solell, du nuage et de l'arc-en-ciel.

\section{Le soleil.}

Les données liées à la représentation du soleil sont présentées sous cinq versions. Au stade pré-schématique, on retrouve le cercle et le cercle avec rayons. Le soleil en coin traduit davantage le stade schématique, tandis que le soleil en triangle et la seule présence de rayons s'insèrent au stade de réalisme visuel.

184 manifestations de cette forme ont été répertoriées sur le total des 399 peintures, c'est-à-dire que $31 \%$ des productions étaient ensoleillées. Le tableau 8 montre qu'une forte proportion des enfants ont utilisé les traits distinctifs du stade pré-schématique. Le nombre le plus important de manifestations, soit $47,5 \%$, est répertorié au stade de réalisme visuel, il s'agit du soleil en triangle. 
TABLEAU 8

Représentation de la forme soleil

\begin{tabular}{|c|c|c|}
\hline & $\begin{array}{l}\text { Nombre d'enfants } \\
\text { ayant utillsé } \\
\text { cette représentation }\end{array}$ & $\begin{array}{l}\text { Nombre de fols } \\
\text { que la représentation } \\
\text { est utillsée }\end{array}$ \\
\hline $\begin{array}{c}0 \\
0 \\
0 \\
0\end{array}$ & $\begin{array}{c}17 \\
6 \\
6 \\
21 \\
0\end{array}$ & $\begin{array}{r}48 \\
10 \\
7 \\
59\end{array}$ \\
\hline
\end{tabular}

Plusieurs autres manifestations originales ont été relevées. II s'agit du soleil couchant à l'horizon, du soleil qui perce entre les nuages, du soleil encerclé et de la présence de l'effet du soleil sur le tableau. Une bonne partie du groupe réalise donc la forme soleil selon les attentes correspondant au stade de réalisme visuel.

\section{Le nuage.}

Le dessin du nuage est observé à partir du type d'application fait avec le pinceau et à partir de la représentation réelle ou irréelle de la couleur d'un nuage.

A la lecture du tableau 9, on observe une utilisation importante d'un trait distinctif propre au stade de réalisme visuel. En effet, le nuage est souvent représenté sans contour traduisant ainsi la maîtrise que les enfants ont acquise vis-à-vis cette forme. Les traits distinctifs associés au stade pré-schématique, soit le trait contour seul et contour différent du contenu sont. également représentés en nombre important. Dans les tableaux où ce type de manifestations a été relevées, le nuage sert de 
remplissage de l'espace feuille plus que d'expression de la forme pour ce qu'elle signifie.

TABLEAU 9

Représentation de la forme nuage

\begin{tabular}{|l|c|c|}
\hline Traits dlstinctifs & $\begin{array}{c}\text { Nombre d'enfants } \\
\text { ayant utllisé } \\
\text { cette représentation }\end{array}$ & $\begin{array}{c}\text { Nombre de fols } \\
\text { que la représentation } \\
\text { est utilisée }\end{array}$ \\
\hline Contour seul & 11 & 27 \\
Contour $\neq$ contenu & 9 & 25 \\
Contour=contenu & 0 & 0 \\
Pas de contour & 16 & 54 \\
Couleur réelle & 5 & 9 \\
Couleur irréelle & 21 & 87 \\
\hline
\end{tabular}

Si l'on observe maintenant le choix de couleurs que les enfants ont priorisé dans la réalisation du nuage, on s'aperçoit qu'il est irréel, c'est-à-dire qu'il ne traduit pas ce que l'enfant voit dans la réalité.

II y a donc un nombre semblable de nuages représentés au stade pré-schématique et au stade de réalisme visuel, mais cette manifestation occupe l'espace feuille différemment.

\section{L'arc en ciel}

L'arc-en-ciel se vérifie par sa représentation réelle ou irréelle des couleurs d'un arc-en-ciel, par l'observation de superposition de couleurs, par la présence de fondue dans les couleurs, par le nombre de couleurs exprimées et enfin, par la répétition de couleurs. 
Ce groupe de pré-adolescents ne semble pas accorder d'importance à l'arc-enciel dans les tableaux qu'ils ont faits. En effet, seulement huit d'entre eux ont dessiné cette forme. Ces résultats nous portent à croire que les enfants se situent davantage dans les stades qui les caractérisent, parce que traditionnellement, le dessin de larc-en-ciel se retrouve plus fréquemment dans les productions de jeunes enfants. De plus, lorsqu'ils ont dessiné cette forme, ils ont utilisé un choix de couleurs appliquées en superposition, trait distinctif propre à leur âge.

\section{La résultats de la représentation de la malson}

Les résultats obtenus concernant la représentation de cette forme sont minces. On peut penser que le faible pourcentage $(6 \%)$ de maisons répertoriées annonce des préoccupations de pré-adolescents qui élargissent leur champ de vision et désirent exprimer des thèmes qui s'inscrivent davantage aux stades qui leur est propre, soit les stades de réalisme visuel et de pseudo-naturalisme.

Si l'on regarde maintenant le tableau 10 , on s'aperçoit que pour dessiner la structure de base de la maison, les enfants ont surtout choisi le trait maison vue de face, trait associé au stade pré-schématique. La façon dont ils ont habillé la maison montre l'utilisation des traits caractéristiques du stade pré-schématique. 
TABLEAU 10

Représentation de la forme maison

\begin{tabular}{|c|c|c|}
\hline Traits distinctifs & $\begin{array}{l}\text { Nombre d'enfants } \\
\text { ayant utllisé } \\
\text { cette représentation }\end{array}$ & $\begin{array}{l}\text { Nombre de fois } \\
\text { que la représentation } \\
\text { est utillsée }\end{array}$ \\
\hline $\begin{array}{l}\text { Porte } \\
\text { Poignée } \\
\text { Fenêtre } \\
\text { Carreaux fenêtre } \\
\text { Rideaux } \\
\text { Cheminée } \\
\text { Fumée } \\
\text { Chemin } \\
\text { Texture mur extérieur } \\
\text { Texture chemin }\end{array}$ & $\begin{array}{r}1 \\
2 \\
10 \\
5 \\
12 \\
6 \\
9 \\
5 \\
3 \\
7 \\
5 \\
5 \\
4 \\
2\end{array}$ & $\begin{array}{r}1 \\
2 \\
14 \\
7 \\
17 \\
6 \\
14 \\
6 \\
3 \\
7 \\
5 \\
6 \\
5 \\
2\end{array}$ \\
\hline
\end{tabular}

Les éléments caractéristiques propres aux enfants de cet âge sont presque absents dans le tableau 10. On en retrouve, par contre, plusieurs à la rubrique "autres". II s'agit de la texture du toit ou de la cheminée, de la sonnette a la porte, de la boîte aux lettres, de la cheminée inclinée, de la fenêtre ronde, de la maison sans ligne de sol, du chemin en diagonale, de la niche, de la maison genre hutte et du gratteciel.

La maison traditionnelle la plus représentée demeure pourtant peu exploitée, et est une forme posée dans le tableau, mais ne semble plus tenir l'importance qu'elle avait chez le jeune enfant. Les portes et les fenêtres sont les seuls traits auxquels une importance est accordée. L'enfant de cet âge paraît préoccupé par d'autres thèmes que celui du gîte et se tourne davantage vers le monde extérieur en traduisant des images que Stern qualifie de résiduelles. II dessine maintenant des maisons de 
tout genre plutôt que sa maison. Peut être est-ce là la marque d'un pas vers les stades de réalisme visuel et pseudo-naturaliste.

\section{Les résultats de la représentation de l'arbre}

21 participants ont peint l'arbre pour un total de 123 manifestations concernant le sujet, soit $30,8 \%$. Les résultats du tableau 11 montrent que la plupart des enfants ont acquis la représentation de cette forme au stade de réalisme visuel, stade qui leur est attribuẻ d'après leur âge. lis ont représenté le feuillu et le conifère de façon sophistiquée. La présence de l'écorce de l'arbre montre également un souci de texture associé à ce stade.

TABLEAU 11

Représentation de la forme arbre

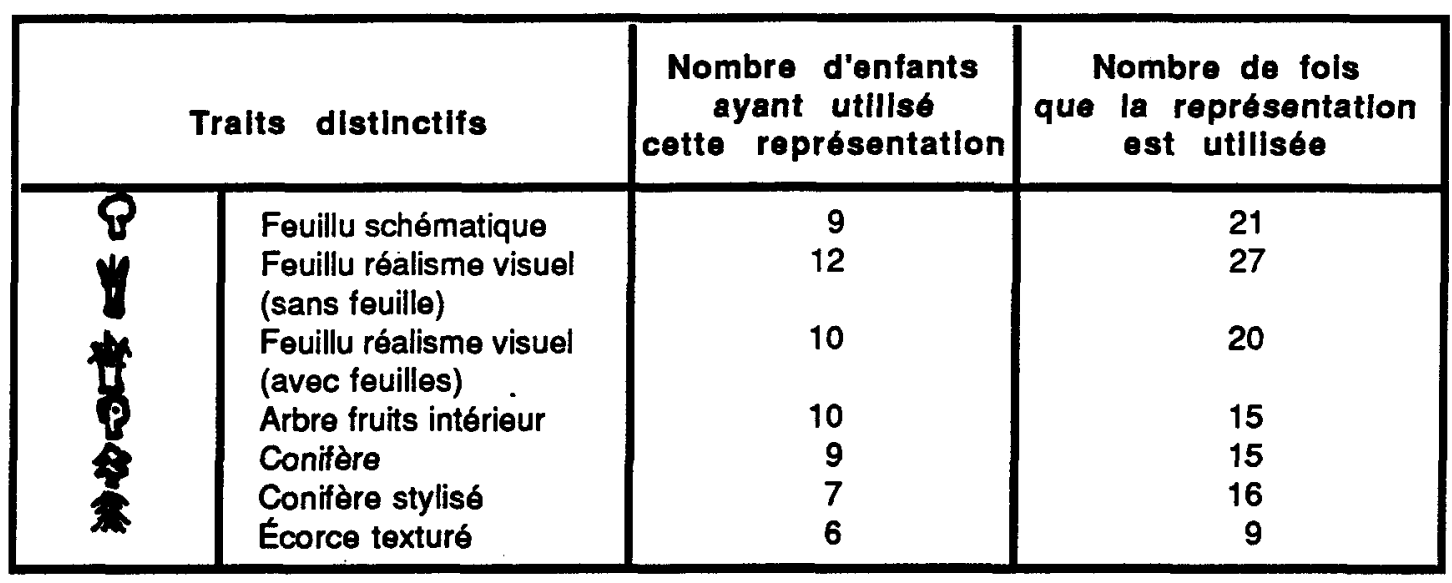

Si l'on regarde maintenant l'utilisation de la couleur réelle dans la présentation de cette forme, on s'aperçoit que c'est un appui pour l'enfant dans son cheminement vers le réalisme visuel. Ces résultats portent à croire que dans l'ensemble, ce 
groupe d'enfants représente l'arbre avec les traits distinctifs associés aux stades de réalisme visuel et de pseudo-naturalisme.

Plusieurs autres traits distinctifs s'associant à ce stade ont été notés. Parmi les plus révélateurs, il y a la nuance dans la couleur du feuillage, la présence d'arbres et de plantes exotiques comme le palmier et le cactus, et enfin, les herbes géantes et les arbres miniatures.

Pour terminer cette partie des résultats, il est intéressant d'observer l'ensemble des formes représentées par les pré-adolescents.

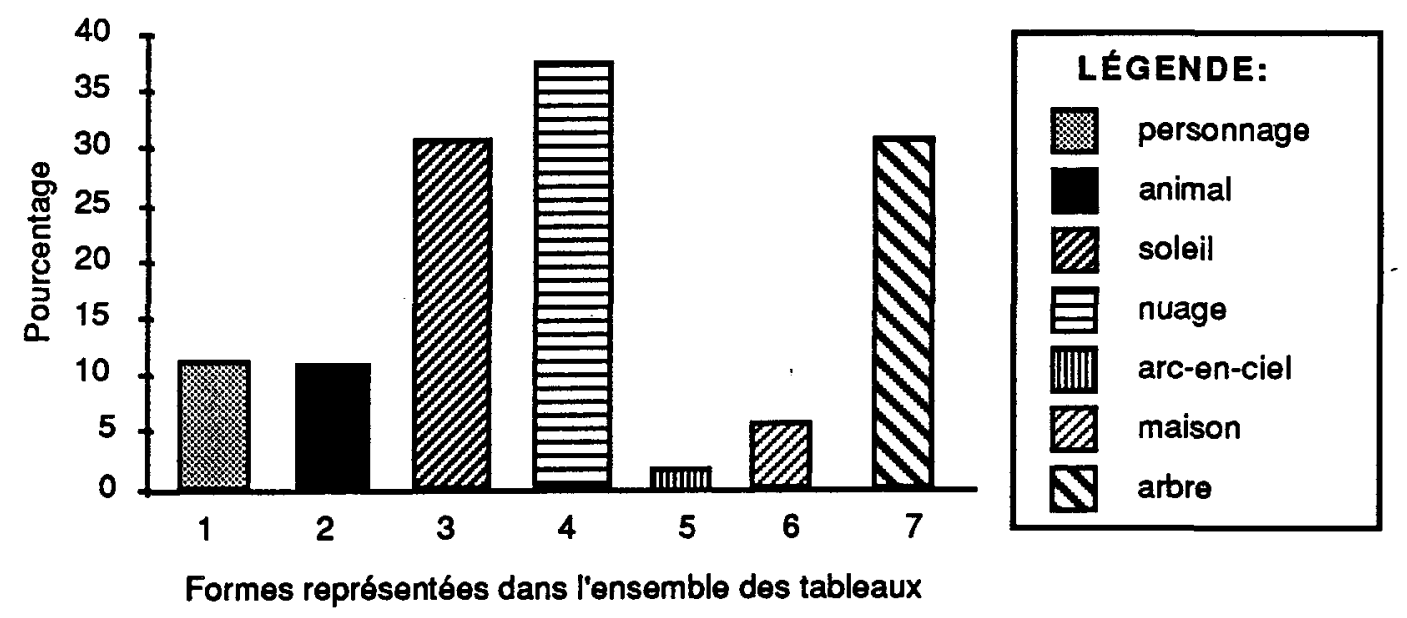

Figure 4: Pourcentage de la variété des formes représentées dans l'ensemble des tableaux.

La figure 4 permet de voir dans quel pourcentage les enfants ont représenté chacune des formes retenues pour l'analyse. Le pourcentage élevé de représentation du soleil, du nuage et de l'arbre situe les productions des enfants dans une entrée dé- 
finitive aux stades propres à leur âge. De plus, ces formes ont été rendues avec un souci du réel lié aux stades réalisme visuel et pseudo-naturaliste.

\section{Les résultats de l'utilisation de la couleur}

Les résultats obtenus concernant l'utilisation de la couleur pour l'ensemble des 399 productions analysées sont présentées au tableau 12 selon le même ordre que celui de la table-palette. Au premier regard, on s'aperçoit que parmi les couleurs les plus utilisées, les couleurs primaires jaune et bleu ont été très populaires. Le vert figure également dans ce registre de popularité. Le blanc s'est retrouvé sur plusieurs productions, couleur permettant la nuance, manifestation souvent explorée au stade du réalisme visuel.

Les mélanges utilisés au nombre de 189 sont surtout le gris, le rose, le vert pâle, le bleu pâle, l'oranger et le lilas.

TABLEAU 12

Utillsation de chacune des couleurs

\begin{tabular}{|c|c|c|c|c|c|c|}
\hline COULEUR & Noir & $\begin{array}{c}\text { Terre de } \\
\text { Slenne }\end{array}$ & Rouge & Ocre & $\begin{array}{c}\text { Jaune } \\
\text { chrome } \\
\text { clailr }\end{array}$ & $\begin{array}{c}\text { Vert } \\
\text { anglals }\end{array}$ \\
\hline Nombre & 26 & 13 & 30 & 68 & 272 & 282 \\
\hline COULEUR & $\begin{array}{c}\text { Bleu } \\
\text { outremer }\end{array}$ & $\begin{array}{c}\text { Terre } \\
\text { ombre } \\
\text { brálé }\end{array}$ & $\begin{array}{c}\text { Jaune } \\
\text { de } \\
\text { chrome }\end{array}$ & Blanc & $\begin{array}{c}\text { Mélan- } \\
\text { ges }\end{array}$ & \\
\hline Nombre & 221 & 141 & 28 & 153 & 189 & \\
\hline
\end{tabular}

Le nombre de couleurs et le nombre de mélanges que les enfants ont utilisés dans la réalisation de chacun des tableaux, permettent de lire au tableau 13 que la 
majorité des productions offre une variété de trois à sept couleurs, ce qui révèle un souci de précision dans la représentation des formes, souci associé au stade schématique et au stade de réalisme visuel. Les enfants utilisent les couleurs de façon variée, s'associant ainsi au stade qui leur est attribué.

TABLEAU 13

Le nombre de couleurs utilisées dans chacune des peintures

\begin{tabular}{|c|c|c|}
\hline $\begin{array}{c}\text { Nombre de couleurs } \\
\text { utillsées }\end{array}$ & $\begin{array}{c}\text { Nombre de dessins } \\
\text { réallsés }\end{array}$ & $\begin{array}{c}\text { Nombre d'enfants } \\
\text { ayant dessiné }\end{array}$ \\
\hline 1 & 9 & 5 \\
2 & 29 & 14 \\
3 & 41 & 20 \\
4 & 60 & 25 \\
5 & 90 & 25 \\
6 & 75 & 24 \\
7 & 62 & 22 \\
8 & 25 & 13 \\
9 & 11 & 10 \\
\hline
\end{tabular}

Si l'on se penche ensuite sur la manière dont les enfants ont choisi de peindre l'ensemble des productions, on note, au tableau 14, un contrôle évident de la forme par l'emploi du trait distinctif sans contour. Les enfants présentent les formes selon les attentes propres à leur åge.

TABLEAU 14

Utilisation de la couleur par rapport aux quatre tralts distinctifs.

\begin{tabular}{|l|c|c|}
\hline Traits distinctifs & $\begin{array}{c}\text { Nombre d'enfants } \\
\text { ayant utilisés } \\
\text { cette représentation }\end{array}$ & $\begin{array}{c}\text { Nombre de fois } \\
\text { que la représentation } \\
\text { est utilisée }\end{array}$ \\
\hline Contour seul & 15 & 49 \\
Contour $\neq$ contenu & 22 & 67 \\
Contour=contenu & 3 & 3 \\
Pas de contour & 26 & 218 \\
\hline
\end{tabular}


En considérant les résultats du tableau 15, montrant la façon dont la couleur a été appliquée aux volets nuance, couleurs superposées, ombre-lumière, texture, motif et couleurs juxtaposées, éléments rattachés au stade de réalisme visuel et au stade pseudo-naturaliste, on distingue des efforts annonçant l'entrée dans ces stades.

TABLEAU 15

Utilisation de la couleur par rapport aux six traits distinctifs.

\begin{tabular}{|l|c|c|}
\hline Traits distinctifs & $\begin{array}{c}\text { Nombre d'enfants } \\
\text { ayant utilisés } \\
\text { cette représentation }\end{array}$ & $\begin{array}{c}\text { Nombre de fois } \\
\text { que la représentation } \\
\text { est utillsée }\end{array}$ \\
\hline Nuance & 12 & 30 \\
Couleurs superposées & 16 & 70 \\
Ombre-lumière & 2 & 6 \\
Texture & 25 & 161 \\
Motif & 11 & 28 \\
Couleurs juxtaposées & 24 & 167 \\
\hline
\end{tabular}

\section{Les résultats de l'organisation de l'espace feullle.}

Les résultats relatifs à l'organisation de l'espace feuille révèlent, au tableau 16, que les enfants n'utilisent que très peu de traits distinctifs propres au stade pré-schématique.

TABLEAU 16

Utilisation des bléments composant l'organlsation de l'espace au stade pré-schématique.

\begin{tabular}{|l|c|c|}
\hline Traits distinctifs & Nombre d'éléments & Nombre d'enfants \\
\hline Éléments verticaux & 15 & 6 \\
Suspension des éléments & 59 & 16 \\
Forme close-contenant & 66 & 20 \\
\hline
\end{tabular}


En effet, parmi les éléments notés à ce stade, la forme de base close-contenant est la plus populaire mais est exprimée de façon sophistiquée, se situant ainsi à un stade plus évolué. La présence d'éléments en suspension ne s'explique pas non plus de la même manière qu'au stade auquel elle est habituellement associé. Les éléments sont en suspension, mais dans la volonté de traduire la texture, l'abstrait. Elle dénote plutôt la présence du souci de l'aspect texture mentionné au stade de réalisme visuel.

Si l'on tient compte maintenant des éléments composant le stade schématique (tableau 17), on s'aperçoit qu'ils représentent le plus grand nombre de traits observés dans l'organisation de l'espace feuille. L'ordre dans les éléments est celui qui a été le plus noté. L'utilisation régulière de cette caractéristique montre que les enfants ont acquis la notion d'ordre dans la représentation qu'ils font de l'environnement.

Le remplissage est la seconde manifestation la plus souvent notée. II semble qu'à cet âge, les enfants occupent et exploitent l'environnement en cherchant à élargir leur connaissance du monde et ils le traduisent par le remplissage de la feuille et le contrôle de l'espace feuille.

La répétition automatique a été utiliséé à plusieurs reprises par 25 participants. Elle était traduite de façon stéréotypée (oiseaux) afin de couvrir la feuille ou de manière à annoncer le souci du motif. Ce souci du motif, exprimé par la répétition automatique, est une manifestation qui peut être davantage associée au stade de réalisme visuel qu'au stade schématique. 
TABLEAU 17

Utilisation des éléments composant l'organisation de l'espace stade schématlque

\begin{tabular}{|l|c|c|}
\hline \multicolumn{1}{|c|}{ Traits distinctifs } & Nombre d'éléments & Nombre d'enfants \\
\hline Répétition automatique & 187 & 25 \\
Inclinaison & 7 & 5 \\
Encadrement & 9 & 9 \\
Remplissage & 254 & 26 \\
Ordre des éléments & 306 & 26 \\
Ligne de sol droite & 117 & 24 \\
Rabattement & 28 & 11 \\
Perspective plan & 154 & 25 \\
Transparence & 6 & 6 \\
Diff. séquences-même endroit & 0 & 0 \\
Diff. actions-temps diff. & 0 & 0 \\
Ligne de ciel & 26 & 16 \\
Exagération de l'objet & 69 & 19 \\
Espace sol et ciel & 42 & 20 \\
Alignement des éléments & 33 & 13 \\
Alignement bas feuille & 163 & 25 \\
Perspective étagée & 22 & 13 \\
\hline
\end{tabular}

L'alignement au bas de la feuille, la ligne de sol, l'espace entre le sol et le ciel et la perspective plan utilisés par la majorité des enfants traduisent leur évolution vers le stade réalisme visuel et présente une compréhension de l'espace, une préoccupation de la représentation de l'horizon.

L'objet dessiné a été exagéré par 19 enfants. Cette manifestation est reliée à la caricature qui se doit d'exagérer pour être réussie et qui exprime bien une attitude fréquente chez les pré-adolescents.

Ce tableau (17) montre également que la ligne de ciel, la transparence, le rabattement, l'encadrement et l'inclinaison sont peu représentés puiqu'ils sont des 
manifestations attendues en début de ce stade. En effet, à mesure que l'enfant avance vers le réalisme, il abandonne ce type de représentation.

Les différentes séquences au même endroit et les différentes actions en temps différents n'ont pas été observées, peut-être à cause de l'absence du choix de thème représenté par la bande dessinée, type de représentation qui s'associe à cet élément.

Les traits d'alignement des éléments et perspective étagée sont peu représentés. Enfin, des données (tableau 17) montrent à quel point les enfants complexifient leur perception de l'environnement et indiquent l'acquisition certaine des caractéristiques du stade schématique.

Au tableau 18, les enfants présentent une perception organisée de la compréhension de l'espace en utilisant des mécanismes raffinés, précisant ainsi une entrée au stade de réalisme visuel.

TABLEAU 18

Utilisation des éléments composant l'organisation de l'espace au stade de réalisme visuel

\begin{tabular}{|c|c|c|}
\hline Tralts distinctifs & Nombre d'éléments & Nombre d'enfants \\
\hline $\begin{array}{l}\text { Ciel rejoint sol } \\
\text { Ligne de sol courbe } \\
\text { Juxtaposition } \\
\text { Symétrie } \\
\text { Perspective vol d'oiseau } \\
\text { Perspective polygone }\end{array}$ & $\begin{array}{r}95 \\
71 \\
17 \\
74 \\
32 \\
4\end{array}$ & $\begin{array}{r}19 \\
22 \\
8 \\
24 \\
18 \\
1\end{array}$ \\
\hline
\end{tabular}


Au stade pseudo-naturaliste, quatre enfants ont utilisé le trait distinctif objets éloignés réduits, pour un total de quatre manifestations. Le peu de représentations répertoriées ne signifie pas que ces enfants sont en retard sur leur âge puisque plusieurs traits distinctifs relevés dans l'organisation de l'espace feuille sont autant de pas vers la perspective. Ces traits révèlent que si ce groupe d'enfants avait eu accès régulièrement à un atelier de peinture dans la classe, le processus développemental qu'ils manifestent aurait été plus sophistiqué et aurait rejoint le stade pseudo-naturaliste.

\section{DISCUSSION}

Les résultats obtenus montrent que dans l'ensemble, les enfants utilisent aisément dans leurs dessins les traits distinctifs associés aux stades pré-schématique et schématique. Ils présentent une utilisation moins importante en quantité de traits distinctifs propres au stade de réalisme visuel et au stade pseudo-naturaliste, tels que listés dans les grilles d'analyse. Ils ont tout de même exploré les avenues de ces stades, comme nous le verrons plus loin, mais souvent avec des traits distinctifs différents de ceux retenus dans la présente étude. II semble, comme Stern l'indique, que les images résiduelles se multiplient à cet âge, tout en se complexifiant durant l'évolution des stades et qu'il devient plus difficile de retrouver des traits distinctifs communs à tous les enfants à partir des stades de réalisme visuel et de pseudo-naturalisme. Le relevé de ces traits distinctifs différents permettra de poursuivre l'élaboration de grilles identifiant les stades graphiques des enfants de 11-12 ans de façon plus précise. 
A la lecture des résultats des personnages dessinés, la plupart des éléments constituant le stade pré-schématique sont apparents dans une proportion élevée, à l'exception des éléments jambe et bras, dont la présence est moins fréquente et peut s'expliquer par le choix répété du personnage de la lignée buste ou de la caricature. Ce choix permet à l'enfant de présenter cette forme dans une perspective plus évoluée. En effet, l'enfant n'a plus besoin de montrer tout le personnage, il décide de nous faire voir l'aspect qu'il retient du personnage.

Parmi les éléments susceptibles d'être notés au stade schématique, les éléments cils et orteils sont à peu près absents. L'absence de représentation du cil et le peu de manifestation de la jambe et du pied peuvent être associés au choix de lignée dans lequel le personnage est dessiné. II est souvent traduit en caricature et rarement en portrait réaliste. II n'est donc pas nécessaire de préciser le cil, il devient plus important de grossir le nez et de faire parler la bouche et les yeux. La difficulté d'utilisation des possibilités du pinceau peut également avoir contribué à restreindre la démonstration de cet élément. Les enfants ont pris un certain temps à manipuler le pinceau en exploitant toutes les facettes qu'il offrait. Ils l'ont d'abord utilisé grossièrement et n'ont que partiellement appris à jouer avec la pointe qui dessine le trait fin précisant la forme.

Le peu de présence de représentation dé la main et du doigt s'explique par la grandè difficulté d'exécution de cet aspect du corps humain. En effet, devant ce problème, l'enfant le soustrait tout simplement, sachant que le défi est trop grand et que de toute manière, il ne situe pas l'importance de son message dans cette dimension. 
Deux représentations fréquentes au stade de réalisme visuel sont apparues, il s'agit de l'expression du visage et la longueur des cheveux. Ces représentations marquent l'entrée de l'enfant dans ce stade, et c'est pour lui un moyen de donner un caractère particulier à la personne qu'il désire peindre. Ce n'est plus un personnage mais une personne qui fait partie du monde. II le représente en lui accordant des particularités.

Le costume, quel que soit son genre, de même que les accessoires sont présents avec une importance certaine. Ils permettent à l'enfant de poursuivre l'expression de préoccupations propres aux enfants de cet âge. On sait que la tenue vestimentaire procure souvent un sentiment d'appartenance à cet âge.

La présence d'ongles ne figure presque pas aux résultats, poursuivant ainsi l'évasion de la représentation de la main et du doigt annoncées dans les stades précédents.

Au stade pseudo-naturaliste, parmi les neuf éléments répertoriables, la couleur des cheveux, le style des cheveux ainsi que la coiffure, sont les plus souvent représentés, venant ainsi renforcer les préoccupations déja exprimées. A la lecture de ces résultats, on peut penser que les traits distinctifs les plus importants illustrant cette forme sont le visage, la coiffure et le vêtement.

Le personnage a été expérimenté par plusieurs utilisateurs, quelques-uns seulement ont poursuivi dans l'exploration de ce thème. Est-ce un choix associé à d'autres préoccupations, ou est-ce un choix de dépannage? II est possible de penser 
qu'à cet âge, l'enfant est conscient de la difficulté que soulève la production réaliste de cette forme et voyant le peu de ressemblance qu'il parvient à lui donner, il décide d'explorer d'autres thèmes. De plus, il est important de se rappeler que ces enfants ont peu utilisé ce médium depuis leur passage en classe maternelle. Enfin, les enfants ont en majorité traité ce sujet sans le situer dans un contexte, sans exprimer de gestes ou de mouvements. Ils ont cependant rempli l'espace qui l'entoure. Le personnage demeure rigide et manque de précision. II serait peut-être intéressant de mettre à la disposition des enfants des livres d'art ou encore de prévoir la projection de diapositives en cours d'année afin de les éveiller à la dimension contexte.

L'animal est un sujet qui a davantage été traité de façon fantaisiste, tant au niveau de la forme que de la couleur. Il a été représenté dans l'irréel sous forme de toutou ou de personnage de bandes dessinées. Ce choix montre que le dessin de l'animal du pré-adolescent est différent de celui du jeune enfant. II est traité en caricature, avec humour et présente des préoccupations davantage liées aux pré-adolescents. De plus, les traits distinctifs relatifs à la représentation de la peau et à la position de l'animal montrent bien que les enfants traduisent cette forme avec des moyens propres à leur âge. Le nombre très peu élevé de représentation de l'élément queue ne doit pas être vu comme un déficit, puisque l'animal est souvent dessiné de face et est un choix de représentation qui ne requiert pas la présence de ce trait distinctif. II est possible que la faible représentation d'éléments répertoriés au stade de réalisme visuel et au stade pseudo-naturaliste soit liée au choix irréel d'animal dessiné puisque les traits distinctifs de ces stades relèvent davantage du réel sur la grille. Il serait important de tenir compte de ces résultats afin d'offrir des informations sur l'art de la caricature. 
La manifestation la plus fréquente de la représentation du soleil peut s'associer à un raffinement de la forme close-contenant. II peut être également important de tenir compte du facteur géographique, risquant d'influencer les productions des enfants, afin d'expliquer la grande présence de cette forme. En effet, sachant que la région est montagneuse, les informations visuelles reçues par les enfants au sujet du lever et du coucher du soleil peuvent favoriser cette manifestation du stade réalisme visuel. Le soleil représenté avec un cercle a été noté également en nombre important. Ce trait distinctif est associé au stade pré-schématique, aux schémas de base qui accompagnent l'enfant. II semble qu'il ait été utilisé de cette façon comme un élément indiscutable du sujet exploité, comme un automatisme.

Le nuage est souvent représenté sans contour et témoigne de l'évolution de l'enfant vers le stade de réalisme visuel. L'enfant qui n'a plus besoin de tracer le contour de la forme pour ensuite la remplir, sait maintenant que le contour et le contenu ne font qu'un tout. Le nuage stratus est donc représenté de cette manière. Le nuage cumulus a souvent été dessiné avec contour. II semble que les enfants aient besoin de tracer les limites du nuage, pour ensuite porter leur attention sur l'effet de rondeur que présente ce type de nuage, effet technique parfois difficile à rendre.

Contrairement aux prévisions, l'arc-en-ciel a été très peu exploité comme représentation et n'exprime pas de préoccupations marquées chez l'enfant de cet âge. Il semble que la forme exprimée soit bien comprise par l'utilisation de la juxtaposition, mais les résultats font croire qu'il y a lieu de soustraire cette forme dans le cadre de recherches ultérieures et peut-être la remplacer par les phénomènes naturels (la montagne, le volcan, les chutes, la nuit, les étoiles filantes, ...) qui ont da- 
vantage retenu l'attention de ce groupe d'enfants comme on peut le constater dans les illustrations suivantes.

m

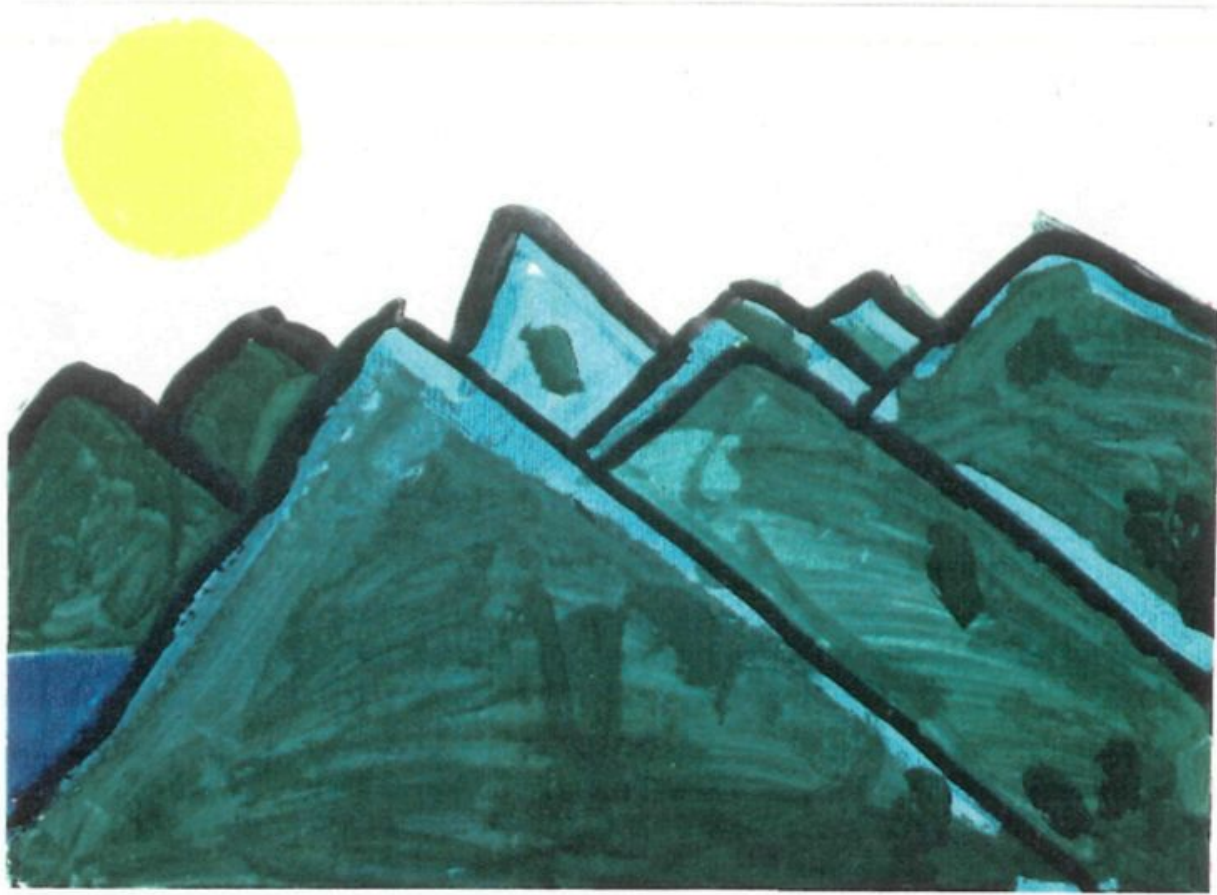

$\checkmark$

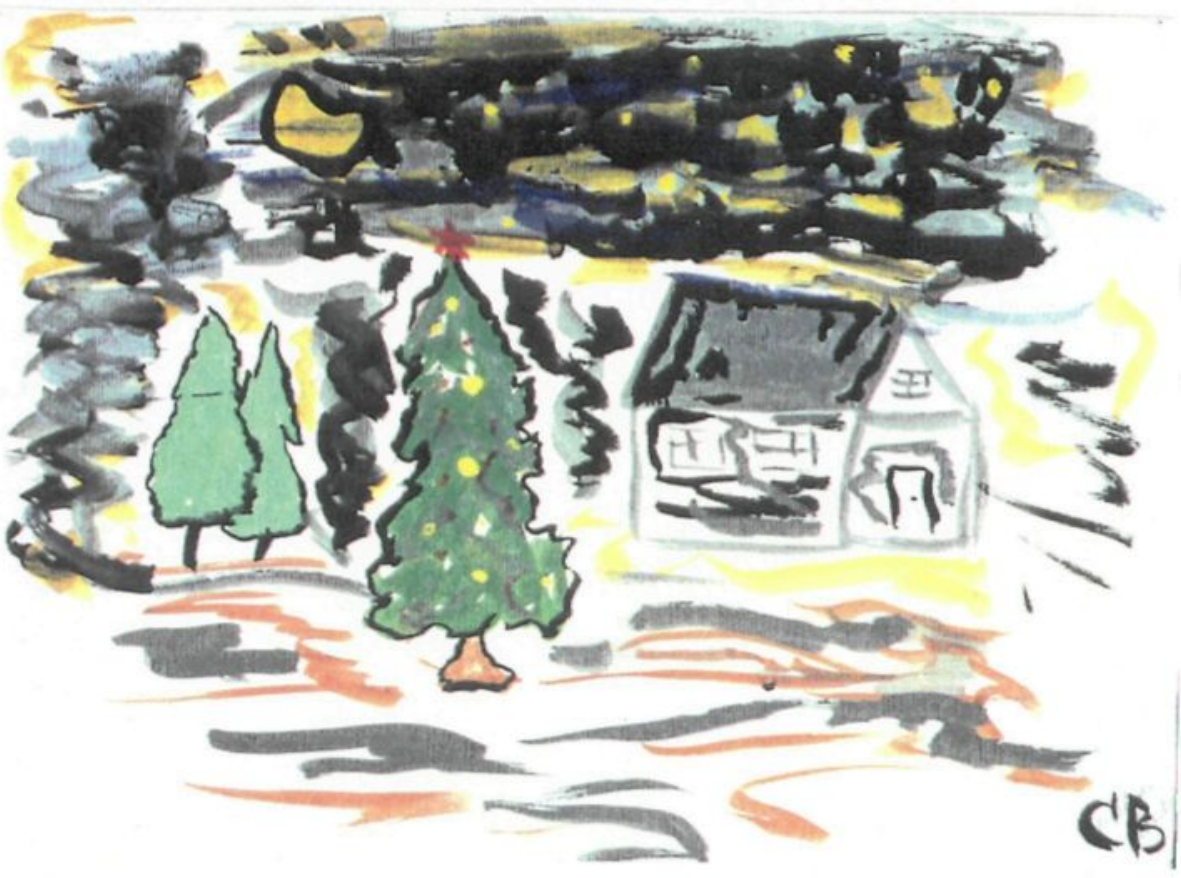




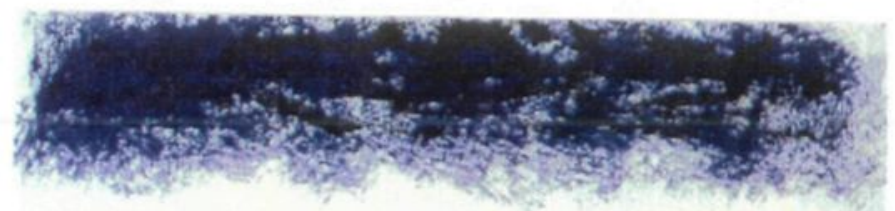

ம

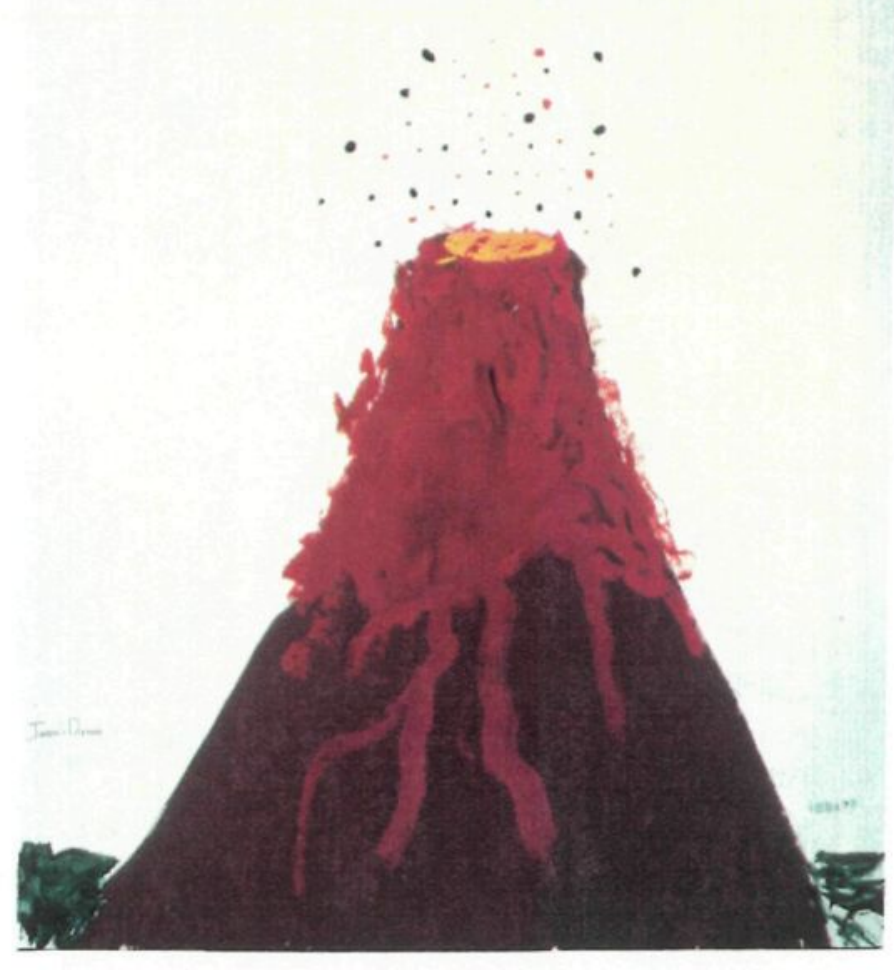

La maison, lorsqu'elle apportait une information supplémentaire a été dessinée à partir de traits distinctifs associés au stade de réalisme visuel. Lorsque cette forme a davantage servi à remplir l'espace, elle a été dessinée à partir de traits distinctifs appartenant au stade pré-schématique. Comme dans le cas du soleil, cette forme a parfois servi à remplir l'espace. On peut questionner ce type de manifestation dans le fait que les enfants n'aient pas grandi avec des possibilités fréquentes de peindre et que leur source d'inspiration en soit diminuée. Ils répètent alors des formes déjà acquises, à titre de technique de dépannage. 
L'arbre, comparativement à la maison, est une forme qui a été développée dans les tableaux observés. En effet, cette forme a servi de thème de travail à quelques enfants qui ont, tout au long de l'année, cherché à rendre un paysage réaliste. Ce genre de productions s'associe aux stades graphiques propres à leur âge. On peut penser que ces manifestations seraient plus nombreuses si les enfants avaient eu tout au long du primaire la possibilité de peindre.

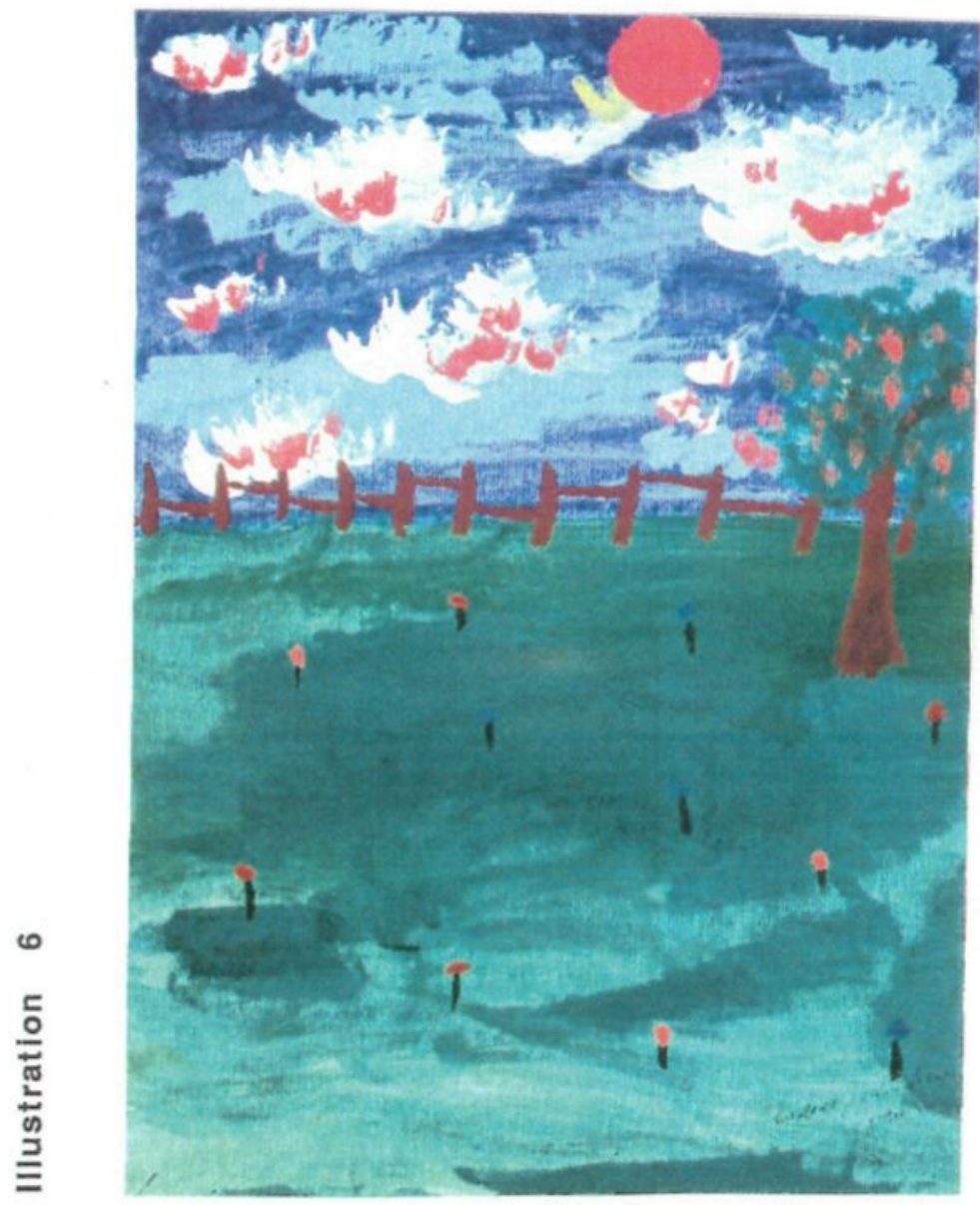


Le choix de couleurs offert à ce groupe d'enfants semble répondre à leurs besoins. L'invention de couleurs a occupé une place considérable dans l'expérience. Elle a permis des discussions et des échanges de recettes. Certains enfants ont même adopté un mélange qui s'est vu utilisé sur une longue période de l'expérience, un peu comme si cette couleur permettait qu'on reconnaisse l'auteur de la production à son choix de couleurs.

Les traits distinctifs associés à l'organisation de l'espace feuille ont été utilisés abondamment. Certaines formes de base habituellement observées au stade préschématique ont été déposées de façon plus ordonnée dans l'espace feuille et sont devenues des images résiduelles, telles que définies par Stern.

Si les enfants avaient eu accès à l'atelier tout au long du primaire, nous aurions sans doute recensé un plus grand nombre de manifestations de ce genre, un plus grand nombre de combinaisons de traits distinctifs reliés à l'organisation de l'espace feuille qui situent l'enfant dans une exploration active du stade de réalisme visuel. En effet, les observations recueillies portent à croire que plusieurs enfants ont peint en utilisant des traits distinctifs de réalisme visuel lorqu'on regarde l'ensemble d'un tableau. 

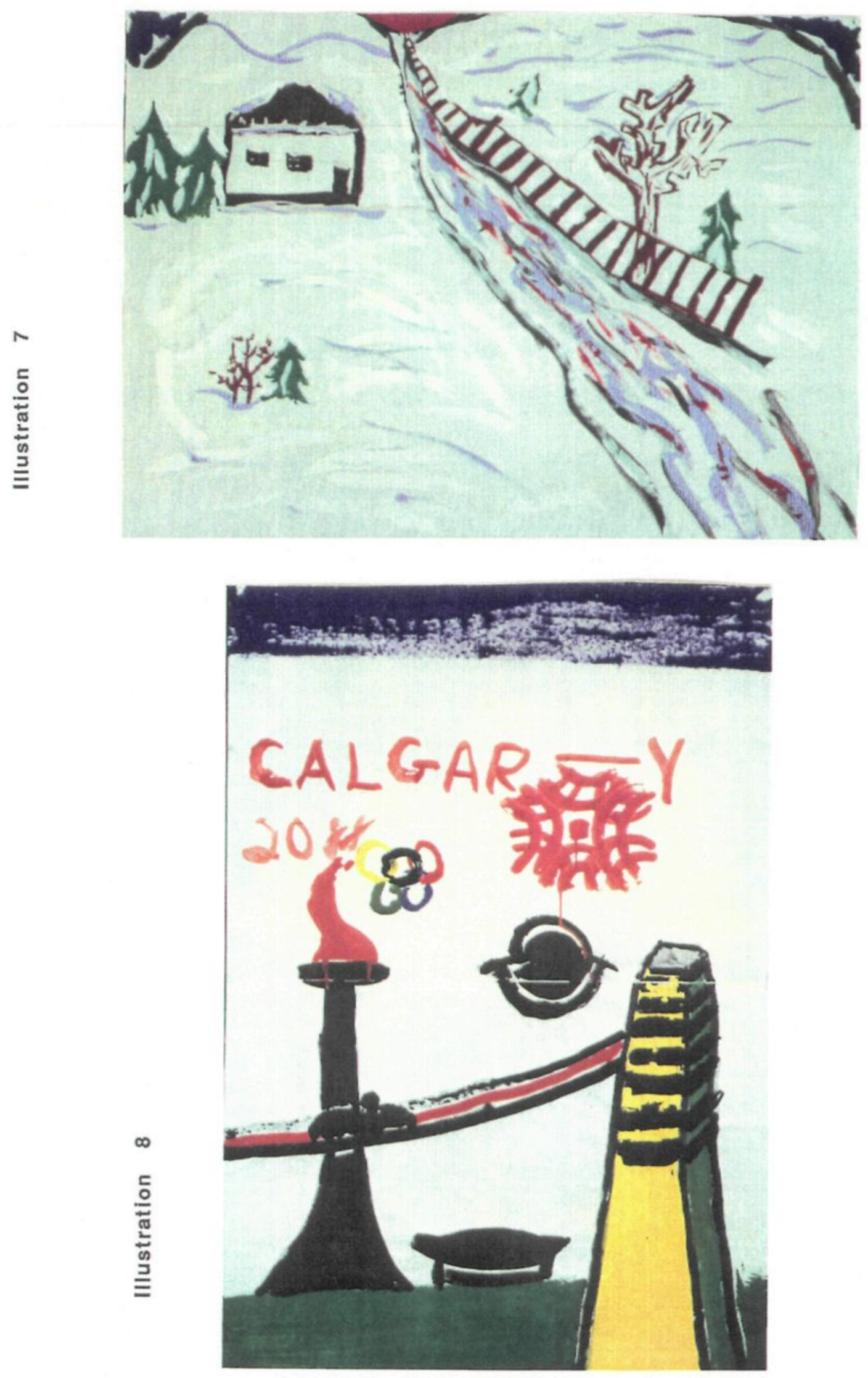
Illustration 10

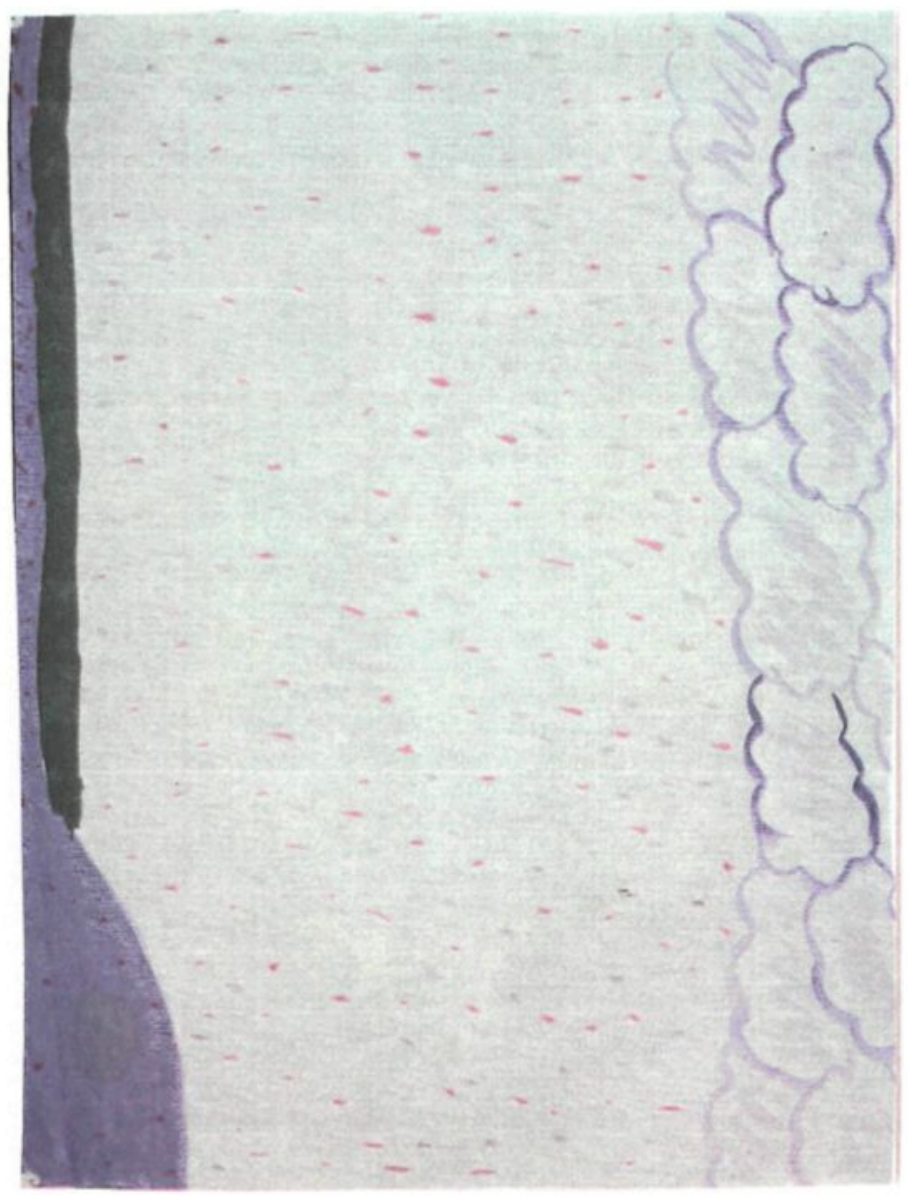

Illustration 9

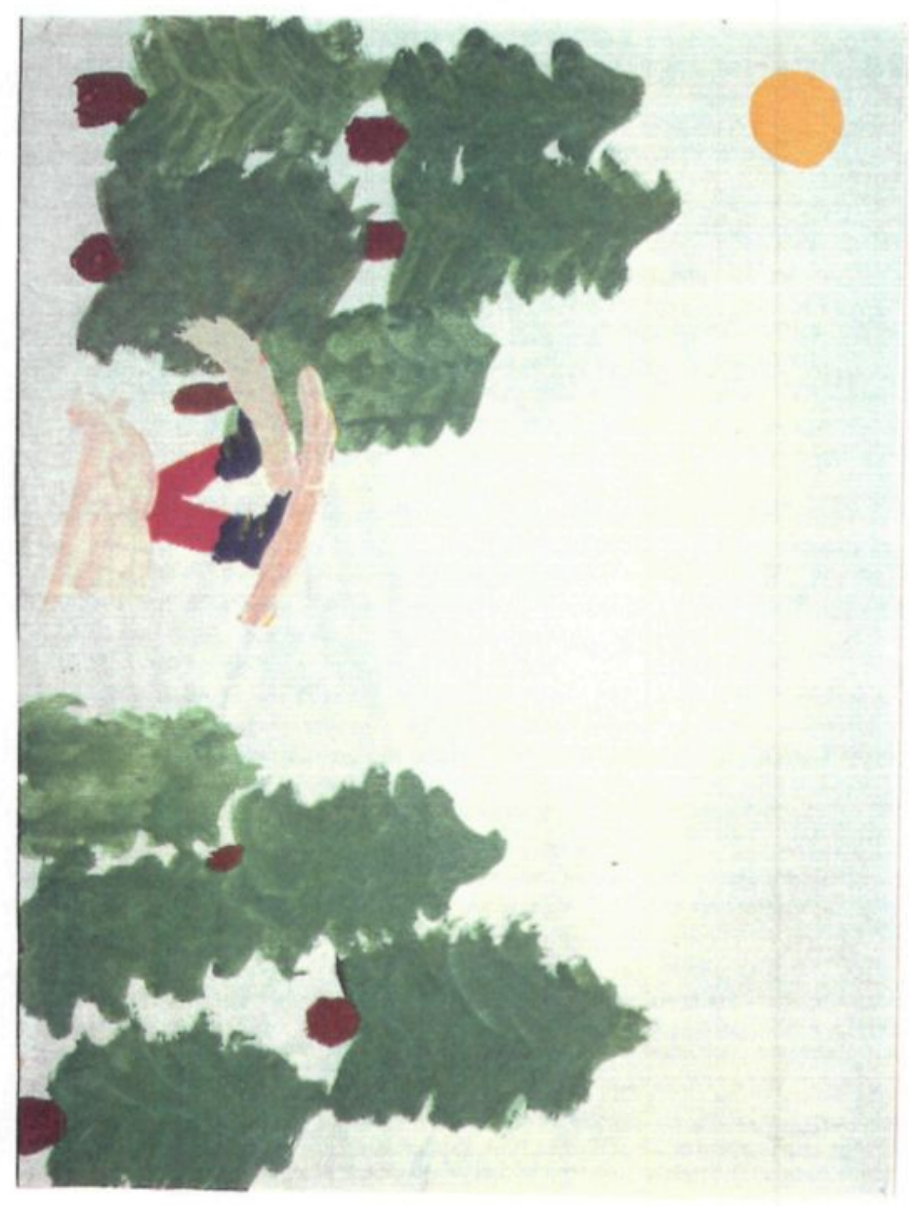


La réalisation de cette expérience était motivée par le désir de répertorier des points de repères le plus précis possible, pour identifier où se situent les enfants de 11-12 ans dans l'acquisition des stades graphiques et pour constater quels sont les traits distinctifs qu'ils utilisent pour constituer une image. II s'agissait également de produire un matériel accessible, facilitant la réalisation continue de l'activité peinture dans une classe.

Les observations qui suivent présentent le taux de fréquentation de l'atelier et l'attitude des enfants dans l'atelier.

\section{LES OBSERVATIONS RELIÉES A L'ORgANISATION MATÉRIELLE}

L'organisation matérielle élaborée pour l'expérience a satisfait les attentes de cette recherche. Les enfants ont eu l'occasion de peindre régulièrement. Ils ont réalisé en moyenne 15 peintures (voir annexe 6).

Durant les premières semaines de l'expérience, seulement quatre enfants pouvaient peindre à chaque séance. Suite à leurs désirs d'une plus grande fréquentation de l'atelier, des panneaux de travail ont été ajoutés, permettant ainsi l'accès à huit enfants. Facile d'utilisation, ce type d'organisation matérielle présente donc une solution au problème vécu en classe et présente également des situations d'intégration de matières, par exemple, au moment de fabrication de décors, d'affiches puisque le matériel est facilement accessible et déplaçable. 
Une faiblesse dans l'utilisation du matériel est retenue. II s'agit du contrôle limité des possibilités du pinceau. Nous aurions pu insister davantage sur cet aspect, mais l'enjeu était délicat puique ces enfants devaient d'abord regagner le goût de peindre. Nous avions convenu en début d'étude d'une intervention verbale limitée. Les modalités de fonctionnement et les directives d'utilisation avaient été présentées clairement, mais n'ont pas été respectées systématiquement. II était parfois difficile d'obtenir, de la part des participants, le comportement souhaité dans le maintien du pinceau, dans le rangement des travaux et de la table-palette. Un encadrement plus structuré et un rappel régulier de la technique d'utilisation du matériel pourraient améliorer cette façette de l'organisation.

Tout au long de l'année, la plupart des participants se sont limités à utiliser une seule feuille. Ceux qui ont exploré des dimensions plus grandes ont réalisé des personnages.

Ce groupe d'enfants, comme déjà mentionné, n'avaient pas peint de façon régulière depuis la maternelle. Ce fait peut être une explication à la faible variété de thèmes qu'ils ont présentés. C'est un peu comme s'ils devaient réapprendre à exprimer des préoccupations à partir de ce médium. La plupart des enfants étaient d'accord et avaient hâte de vivre cette activité. Quelques-uns n'y voyaient pas d'intérêt, éprouvaient des difficultés à choisir un thème de travail, à organiser le temps disponible, un peu comme si, pour eux, le geste était inutile. Par la suite, ces enfants ont trouvé une motivation à l'atelier, mais ont vécu à quelques reprises des pannes d'inspiration. Afin de les stimuler à peindre davantage, deux artistes sont venus vivre une journée en atelier avec le groupe d'enfants pour dire, expliquer et présenter 
leur travail. Les enfants ont ensuite réalisé une activité à partir de la technique utilisée par ces deux artistes.

La majorité des enfants ont réalisés les travaux individuellement, une seule équipe a peint un travail collectif dans le cadre de l'expérience. Durant la période réservée à cette activité, les particiants ont échangé leurs trouvailles en application de peinture, en choix de thèmes et en fabrication de mélanges de couleurs.

\section{CONCLUSION}

L'étude a permis de voir que, même si les tableaux des enfants présentaient davantage de caractéristiques reliées aux stades pré-schématique et schématique, les enfants évoluent en bon nombre vers une appropriation des caractéristiques propres à leur âge. Il est possible d'observer, à partir de la combinaison subtile des traits distinctifs que les enfants ont exprimés, des indices significatifs montrant le processus de construction de stades graphiques plus avancés.

L'étude montre bien qu'il est possible d'organiser un atelier de peinture permanent en classe. Le modèle adapté de la table-palette de Stern, utilisé pour l'expérience, élimine les contraintes d'organisation matérielle rencontrées dans une classe. La table-palette devient un atelier roulant qui offre un matériel de qualité et fait voir aux utilisateurs l'importance de leurs réalisations. Moins longue et plus stable que celle de Stern, c'est un matériel invitant parce que l'enfant passe vite à l'action de peindre. 
L'organisation matérielle privilégiée dans cette étude mérite donc d'être retenue parce qu'elle propose une solution intéressante à l'absence d'atelier de peinture au primaire. Encore est-il nécessaire qu'il y ait une volonté de favoriser le développement du langage plastique dans la personnalité de l'enfant.

Cette étude a permis l'élaboration de grilles d'analyse. Ces grilles identifiaient les traits distinctifs correspondant à chacun des stades graphiques que traversent l'enfant dans sa construction du langage plastique. Tout d'abord basées sur les recherches déjà effectuées par Lowenfeld, Stern, Luquet et Oinga, elles ont permis de songer à l'ajout de nouveaux traits distinctifs pouvant enrichir la lecture des tableaux d'enfants de 11-12 ans. Dans une poursuite éventuelle du sujet, les formes "montagne et volcan" pourront, entre autres, être retenues. Les aspects "présence d'un thème" et "présence de phénomènes naturels" seraient également intéressants à vérifier. Cet outil a permis de situer les tableaux des enfants dans l'échelle des stades graphiques et de répondre à leurs besoins par une organisation matérielle adéquate.

II est souhaitable qu'une étude de ce genre soit entreprise pour l'ensemble des niveaux scolaires, soit de la maternelle à la sixième année afin de vérifier de façon longitudinale l'effet de la présence de l'atelier de peinture sur l'apparition des stades graphiques. II serait également pertinent qu'un perfectionnement soit envisagé pour amener les enseignantes à utiliser des techniques qui privilégient l'expression graphique des enfants. 


\section{RÉFÉRENCES}


DIRECTION GÉNÉRALE DES PROGRAMMES (1986). Guide pédagogique, éveil aux langages artistiques. Québec: M E Q.

DIRECTION GÉNÉRALE DU DÉVELOPPEMENT PÉDAGOGIQUE (1981). Programme d'éducation préscolaire. Québec: M.E.Q.

DIRECTION GÉNÉRALE DU DÉVELOPPEMENT PÉDAGOGIQUE (1981). Programme d'études. Primaire. Art. Québec: M.E.Q.

DIRECTION GÉNÉRALE DU DÉVELOPPEMENT PÉDAGOGIQUE (1982). Répertoire général de la recherche en enseignement des arts au Québec 1960-80, Tome 2, Mémoires et theses. Québec: M.E.Q.

FORTIER-LAVOIE, R. (1984). Apprentissage de la lecture en pédagogie ouverte. Québec: Centre d'intégration de la personne de Québec inc.

GOETZ, E. et ALLEN-K., E. (1983). Early childhood education. Kansas; Aspen publication, University of Kansas.

KUYSECOM, Wim (1976). L'état actuel de l'enseignement des arts plastiques au primaire, mémoire (inédit) présenté à l'Université Concordia.

KRITCHEVSKY, S. et PRESCOTT, E. (1969). Planning environments for young children-physical space. N.Y.: National Association for the education of young children.

LOWENFELD, V. (1968). Speaks on Art and Creativity. London: Ed. W. Lambert Britain.

LOWENFELD, V. (1969). Your child and his art: a guide for parents. London: MacMillan Company.

LOWENFELD, V. (1970). Creative and menthal growth (5th edition). London: MacMillan Company. 
LUQUET, G.H. (1967). Le dessin enfantin. Neuchâtel: Delachaux et Nieslté, Collection Action pédagogique.

NASH, B.C. (1981). The effects of classroom spatial organisation on four and five years old children learning, British Journal of Educational Psychology, vol. 51, pp.144-155.

OZINGA, C. (1969). L'activité créatrice et l'enfant. Québec: P U L.

PHYFE-PERKINO, Elizabeth (1979). Children's behavior in pre-school settings: $A$ review of research concerning the influence of the physical environment. Mémoire présenté à l'University of Massachusetts (inédit).

POMMERLEAU, A. et MALCUIT, G. (1983). L'enfant et son environnement. Québec, Sillery: P.U.Q.

STERN, A. (1959). Aspects et techniques de la peinture d'enfants. Neuchâtel: Delachaux-Niestlé.

STERN, A. (1961). Revue Art Éducateur. Neuchâtel: Delachaux-Niestlé, $n^{\circ} 7$, décembre "La maison image du moi"..

STERN, A. (1963). Le langage plastique. Neuchâtel: Delachaux-Niestlé.

STERN, A. (1963). Le langage plastique. Neuchâtel: Delachaux-Niestlé.

STERN, A. (1966). Une grammaire de l'art enfantin. Neuchâtel: DelachauxNiestlé.

STERN, A. (1967). Entre éducateurs, Réflexions sur l'éducation artistique. Neuchâtel: Delachaux-Niestlé.

STERN, A. (1970). Initiation à l'éducation créatrice. Paris: Éducation nouvelle.

STERN, A. (1976). L'expression ou l'homo-vulcanus. Neuchâtel: DelachauxNiestlé. 
THÉRIAULT, J., DOUCET, M., DOYON, D. et VAN THAM, S. (1987b). "L'exploitation du matériel de l'aire des jeux symboliques à la maternelle", Rapport de recherche, Équipe PRAME, Document \#2, Chicoutimi, UQAC, 124 pages.

THÉRIAULT, J., DOUCET, M., DOYON, D. et VAN THAM, S. (1987d). "Influence de l'organisation matérielle de l'atelier de peinture en classe maternelle sur l'apparition des caractéristiques du ștade graphique correspondant à l'âge de l'enfant", Rapport de recherche, Équipe PRAME, Document \#4, Chicoutimi, UOAC. 


\begin{abstract}
ANNEXE I
Tableau-synthèse des stades graphiques

selon Lowenfeld
\end{abstract}


STADE DU GRIBOUILLAGE (2-4 ANS)

\begin{tabular}{|l|l|l|l|}
\hline Caractéristiques & $\begin{array}{c}\text { Représentation } \\
\text { de la forme }\end{array}$ & $\begin{array}{c}\text { Utllisation } \\
\text { de la couleur }\end{array}$ & $\begin{array}{l}\text { Organlsation } \\
\text { de l'espace }\end{array}$ \\
\hline $\begin{array}{l}\text { Gribouillage en } \\
\text { désordre }\end{array}$ & Aucune & $\begin{array}{l}\text { Sans choix } \\
\text { conscient }\end{array}$ & Aucune \\
\hline $\begin{array}{l}\text { Gribouillage } \\
\text { contrôlé }\end{array}$ & Aucune & $\begin{array}{l}\text { Sans choix } \\
\text { conscient }\end{array}$ & $\begin{array}{l}\text { Expérience } \\
\text { kinesthésique }\end{array}$ \\
\hline $\begin{array}{l}\text { Gribouillage } \\
\text { identifié }\end{array}$ & $\begin{array}{l}\text { Imaginatif, nomme } \\
\text { ce qu'il fait }\end{array}$ & $\begin{array}{l}\text { Pour différencier le } \\
\text { sens des gribouillis, } \\
\text { pour établir relation } \\
\text { entre objets }\end{array}$ & $\begin{array}{l}\text { Représentation } \\
\text { imaginative }\end{array}$ \\
\hline
\end{tabular}


STADE PRÉ-SCHÉMATIQUE (4-7 ANS)

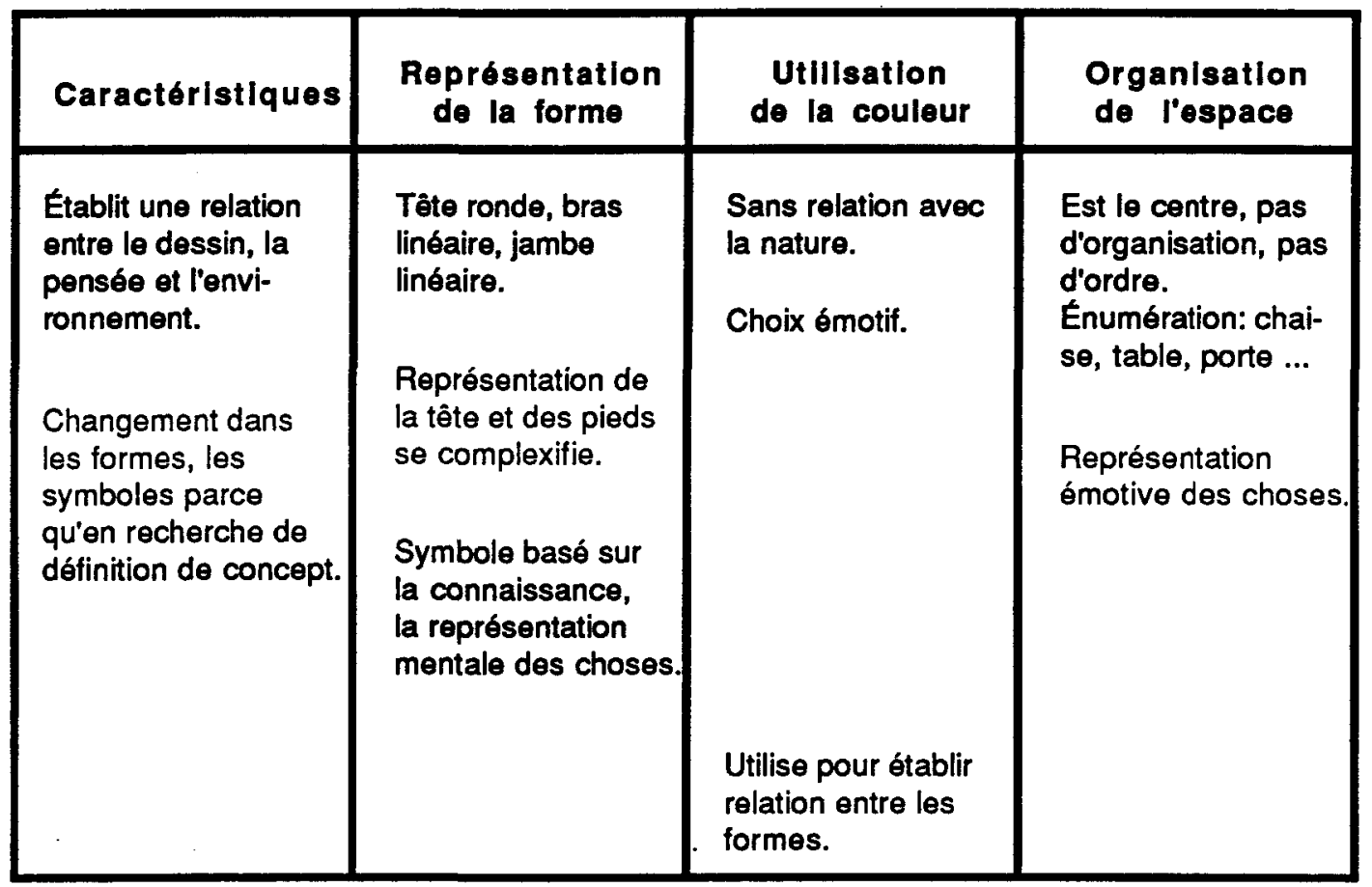


STADE SCHÉMATIQUE (7-9 ANS)

\begin{tabular}{|c|c|c|c|}
\hline Caractéristiques & $\begin{array}{c}\text { Représentation } \\
\text { de la forme }\end{array}$ & $\begin{array}{l}\text { Utillisation } \\
\text { de la couleur }\end{array}$ & $\begin{array}{l}\text { Organisation } \\
\text { de l'espace }\end{array}$ \\
\hline $\begin{array}{l}\text { Formule un concept } \\
\text { défini du personnage } \\
\text { et de l'environnement. }\end{array}$ & $\begin{array}{l}\text { Raffine le schéma } \\
\text { selon l'ajout de } \\
\text { connaissances. }\end{array}$ & $\begin{array}{l}\text { Fait relation entre } \\
\text { couleur et objet, } \\
\text { place les objets en } \\
\text { catégorie: ciel bleu. }\end{array}$ & $\begin{array}{l}\text { Une ligne de sol. } \\
\text { Deux lignes de sol. }\end{array}$ \\
\hline $\begin{array}{l}\text { Bâtit confiance en } \\
\text { soi en répétant for- } \\
\text { mes et symboles. }\end{array}$ & Répétition. & & $\begin{array}{l}\text { Fait partie de l'en- } \\
\text { vironnement: coo- } \\
\text { père. }\end{array}$ \\
\hline $\begin{array}{l}\text { Dessine un schéma, } \\
\text { un symbole de l'objet } \\
\text { réel. } \\
\text { Représente l'idée } \\
\text { qu'il se fait d'un } \\
\text { concept. } \\
\text { Utilise lignes géo- } \\
\text { métriques. }\end{array}$ & $\begin{array}{l}\text { Modifie son expé- } \\
\text { rience et utilise: } \\
\text { - exagération } \\
\text { - omission } \\
\text { - transformation }\end{array}$ & $\begin{array}{l}\text { Varie la couleur } \\
\text { pour exprimer une } \\
\text { émotion. }\end{array}$ & $\begin{array}{l}\text { Espace subjectif: } \\
\text { - rabattement } \\
\text { - plan et élévation } \\
\text { - transparencé } \\
\text { - diff. séquences } \\
\text { d'un même endroit } \\
\text { - diff. actions pla- } \\
\text { cées dans des } \\
\text { temps différents } \\
\text { sur même feuille. } \\
\text { Ordonne. }\end{array}$ \\
\hline
\end{tabular}


STADE DE RéALISME VISUEL (9-12 ANS)

\begin{tabular}{|c|c|c|c|}
\hline Caractérlstlques & $\begin{array}{c}\text { Représentation } \\
\text { de la forme }\end{array}$ & $\begin{array}{l}\text { Utillsatlon } \\
\text { de la couleur }\end{array}$ & $\begin{array}{l}\text { Organisation } \\
\text { de l'espace }\end{array}$ \\
\hline $\begin{array}{l}\text { Age de la bande. } \\
\text { Indépendance } \\
\text { sociale. } \\
\text { Souci du détail, } \\
\text { différences sexuelles } \\
\text { approche de l'horizon }\end{array}$ & $\begin{array}{l}\text { Attention portée aux } \\
\text { vêtements, au sexe, } \\
\text { aux différences gar- } \\
\text { çon-fille. } \\
\text { Approche vers la } \\
\text { réalité: cheveux. }\end{array}$ & $\begin{array}{l}\text { Couleurs objectives, } \\
\text { nuancées ou sub- } \\
\text { jectives et passion- } \\
\text { nées. }\end{array}$ & $\begin{array}{l}\text { Ligne de sol plus } \\
\text { naturelle, } \\
\text { présence de cour- } \\
\text { bes, } \\
\text { ciel nuancé. } \\
\text { Encore rigide. } \\
\text { Deux plans: } \\
\text { - maison derrière } \\
\text { - auto devant } \\
\text { Remplir l'espace. } \\
\text { Ciel rejoint sol. }\end{array}$ \\
\hline
\end{tabular}


STADE DE PSEUDO-NATURALISME (12-14 ANS)

\begin{tabular}{|l|l|l|l|}
\hline Caractéristlques & $\begin{array}{c}\text { Représentation } \\
\text { de la forme }\end{array}$ & \multicolumn{1}{|c|}{$\begin{array}{c}\text { UtIllisation } \\
\text { de la couleur }\end{array}$} & \multicolumn{1}{|c|}{$\begin{array}{c}\text { Organisation } \\
\text { de l'espace }\end{array}$} \\
\hline $\begin{array}{l}\text { Développe intelligen- } \\
\text { ce jusqu'à présent } \\
\text { inconsciente. }\end{array}$ & $\begin{array}{l}\text { Améliore l'action du } \\
\text { personnage. }\end{array}$ & $\begin{array}{l}\text { Nuance se rappro- } \\
\text { che du naturel. }\end{array}$ & $\begin{array}{l}\text { Trois dimensions. } \\
\text { Diminue la taille des } \\
\text { objets éloignés. }\end{array}$ \\
$\begin{array}{l}\text { Approche naturelle. } \\
\text { Aime exagération et } \\
\text { action. }\end{array}$ & $\begin{array}{l}\text { Sertionsie des pro- } \\
\text { portions sur l'expres- } \\
\text { sion. }\end{array}$ & $\begin{array}{l}\text { Les utilise aussi } \\
\text { de façon émotive. }\end{array}$ & $\begin{array}{l}\text { Horizon présent. } \\
\text { Environnement mani- } \\
\text { festé seulement lors- } \\
\text { que signifiant. }\end{array}$ \\
\hline
\end{tabular}


ANNEXE II

Modèles de schémas

proposés par A. Stern 


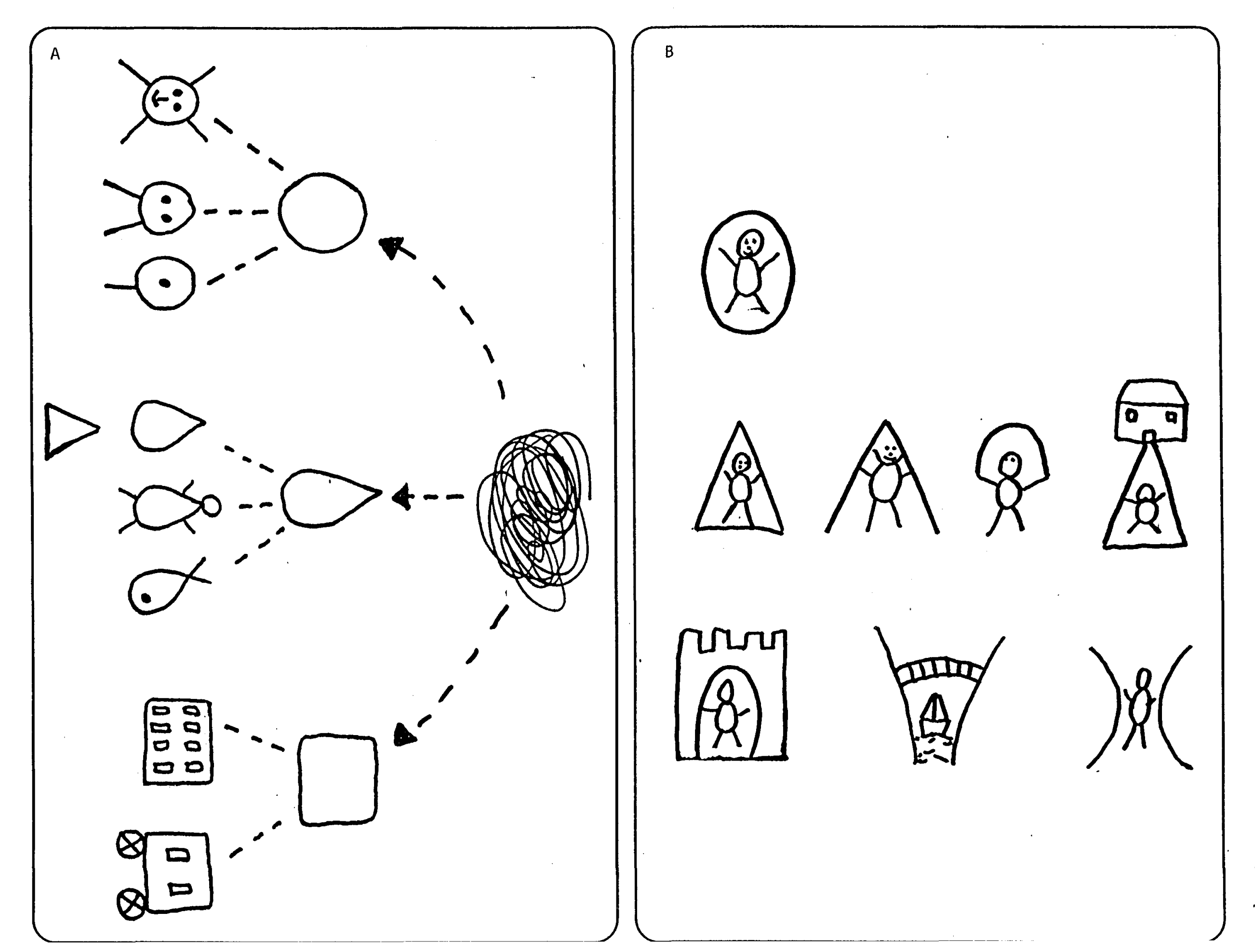




$$
\text { (3) }
$$




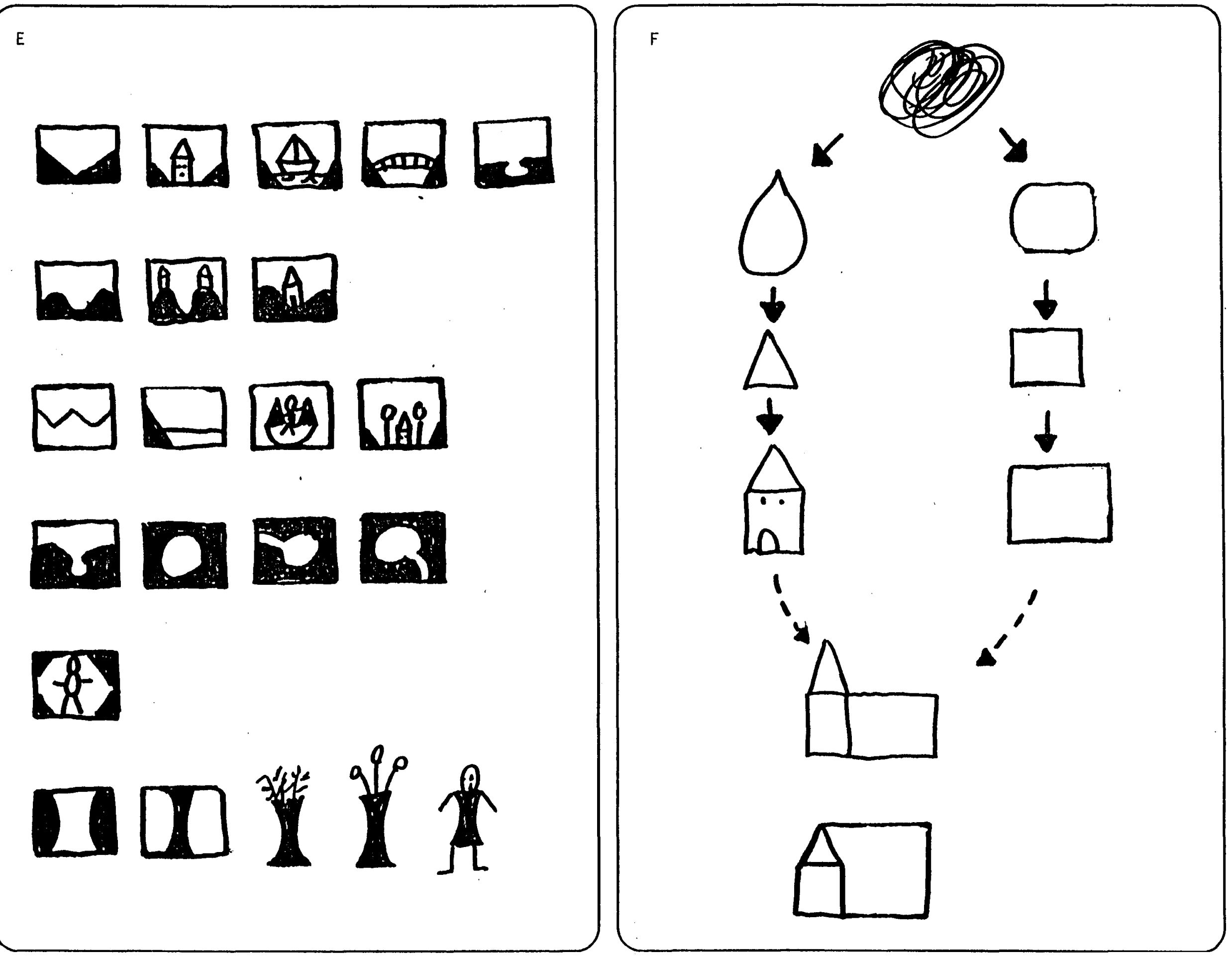




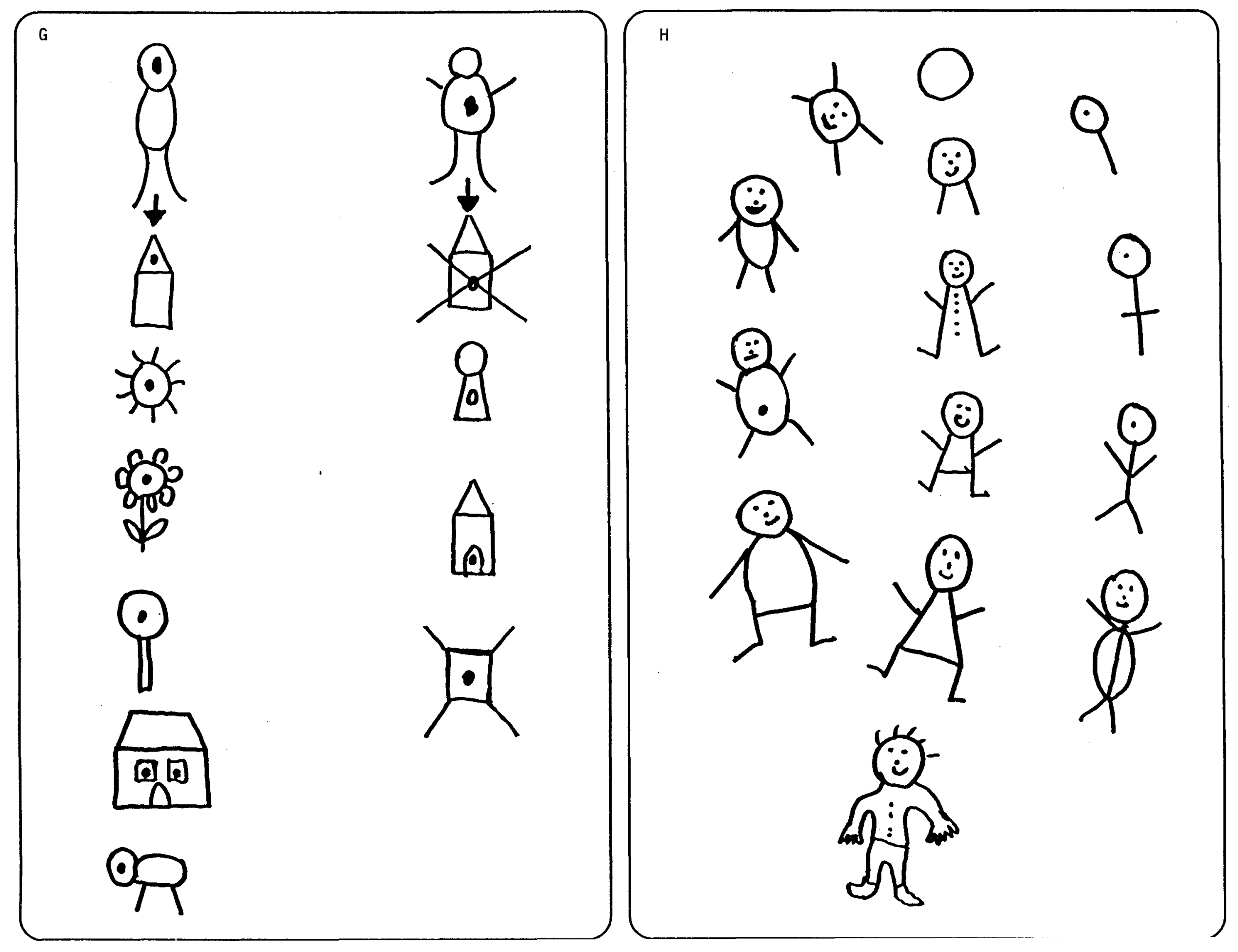




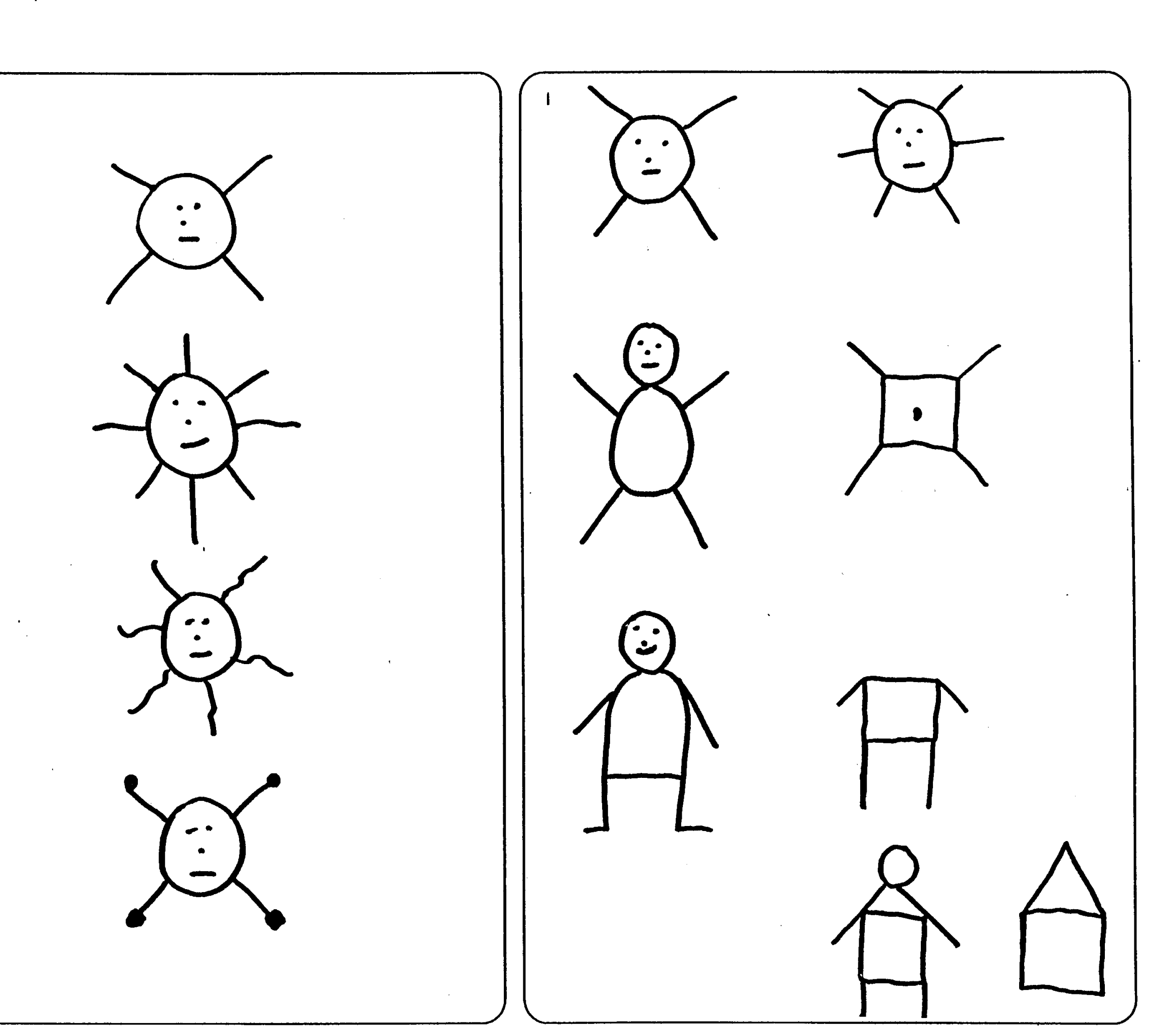




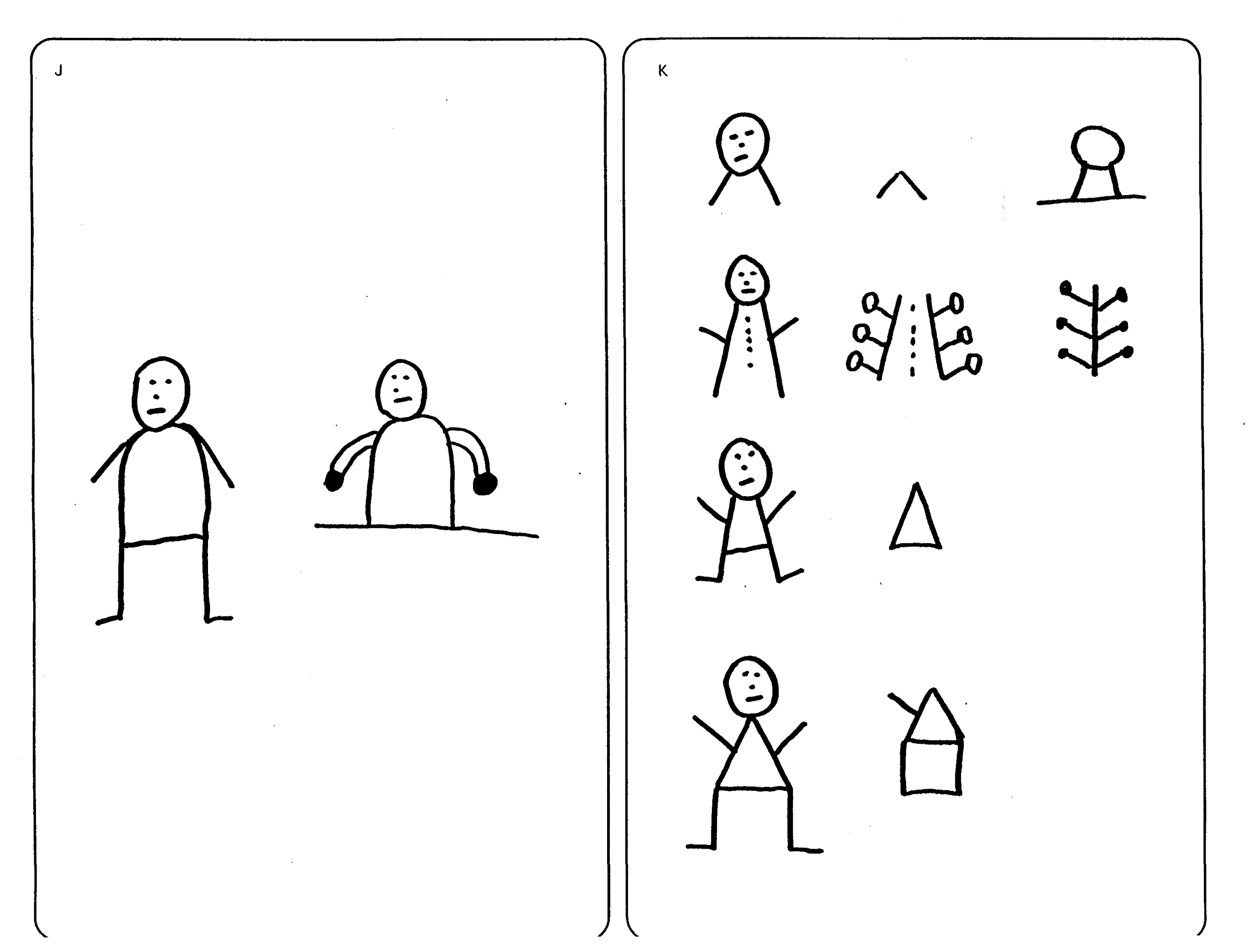




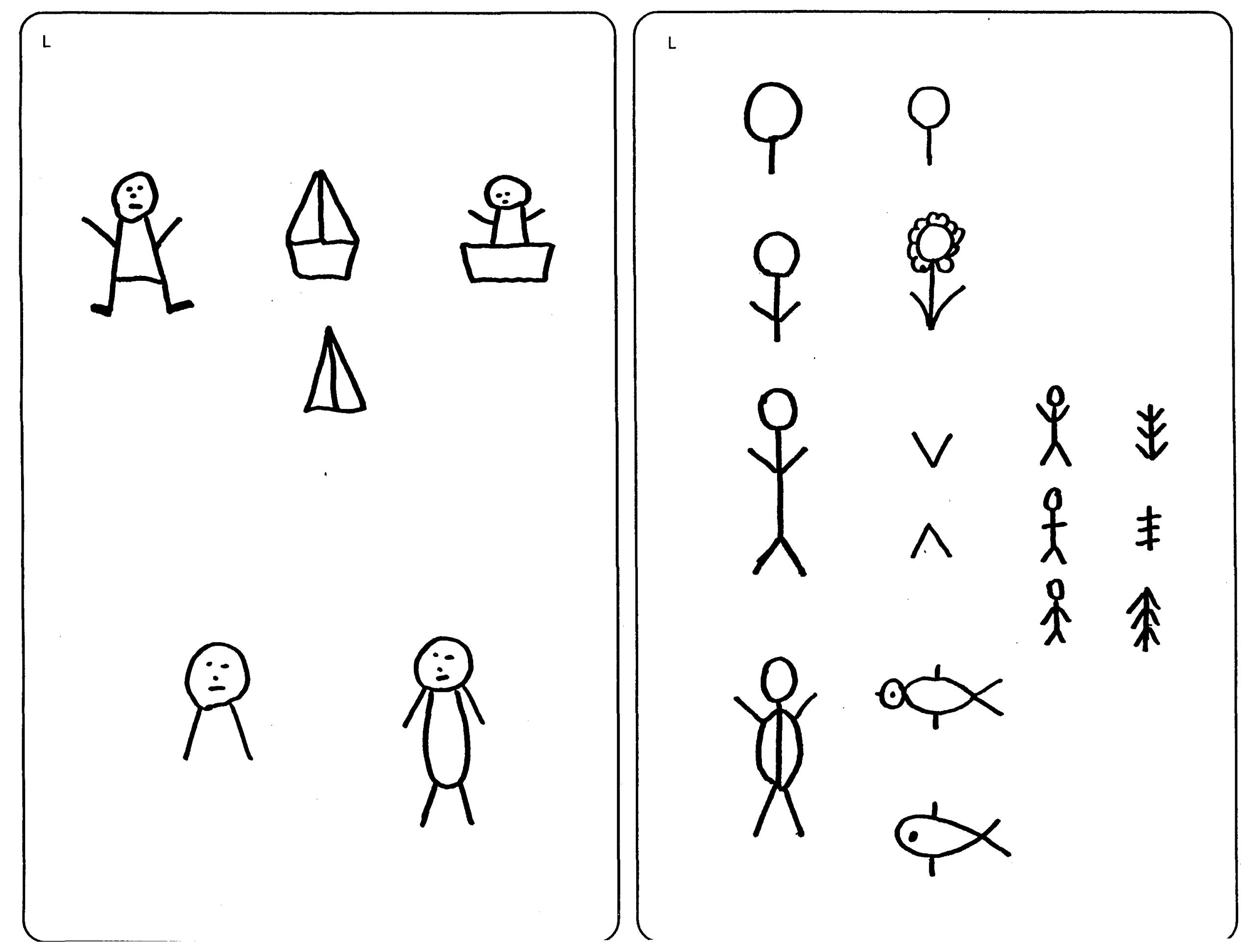




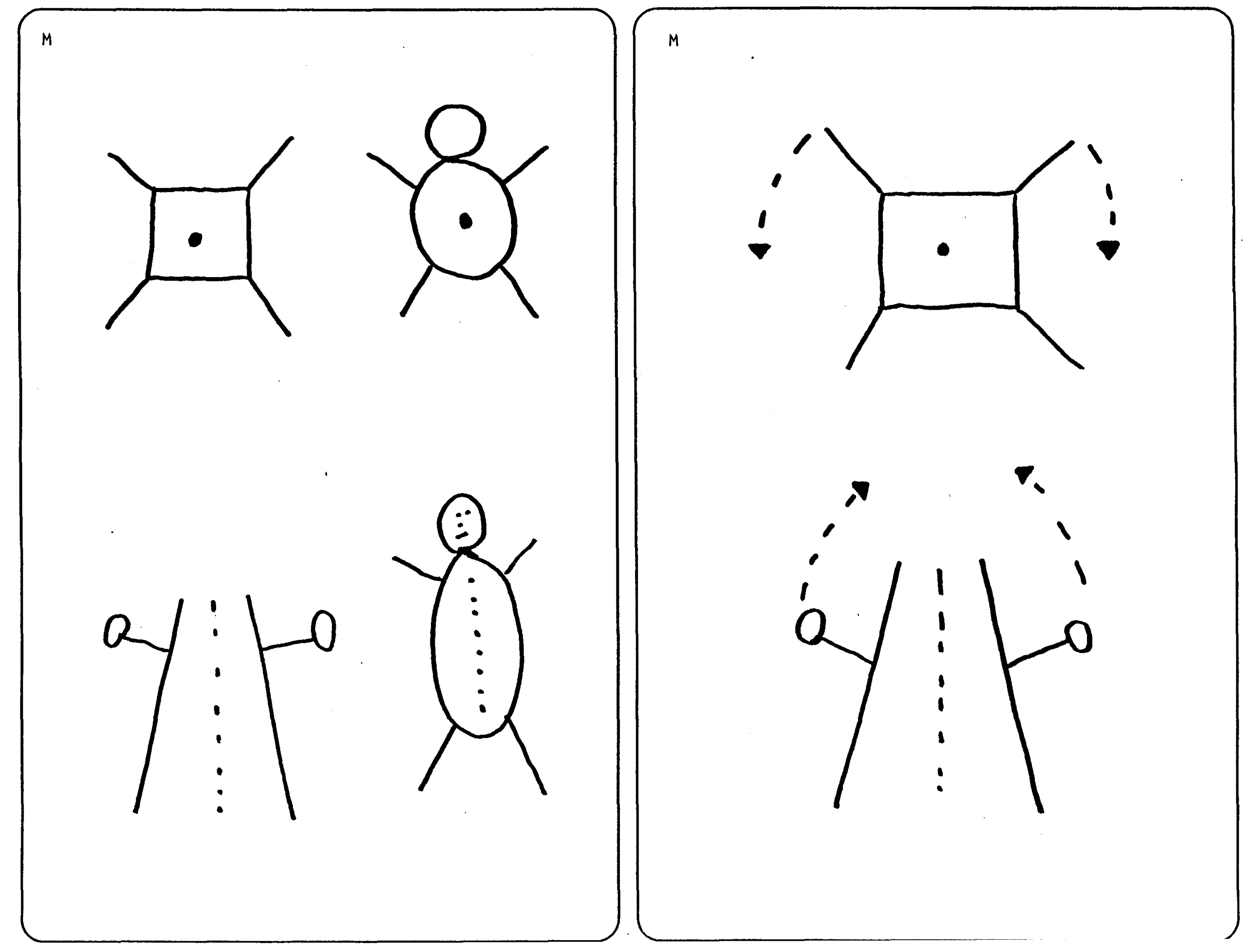




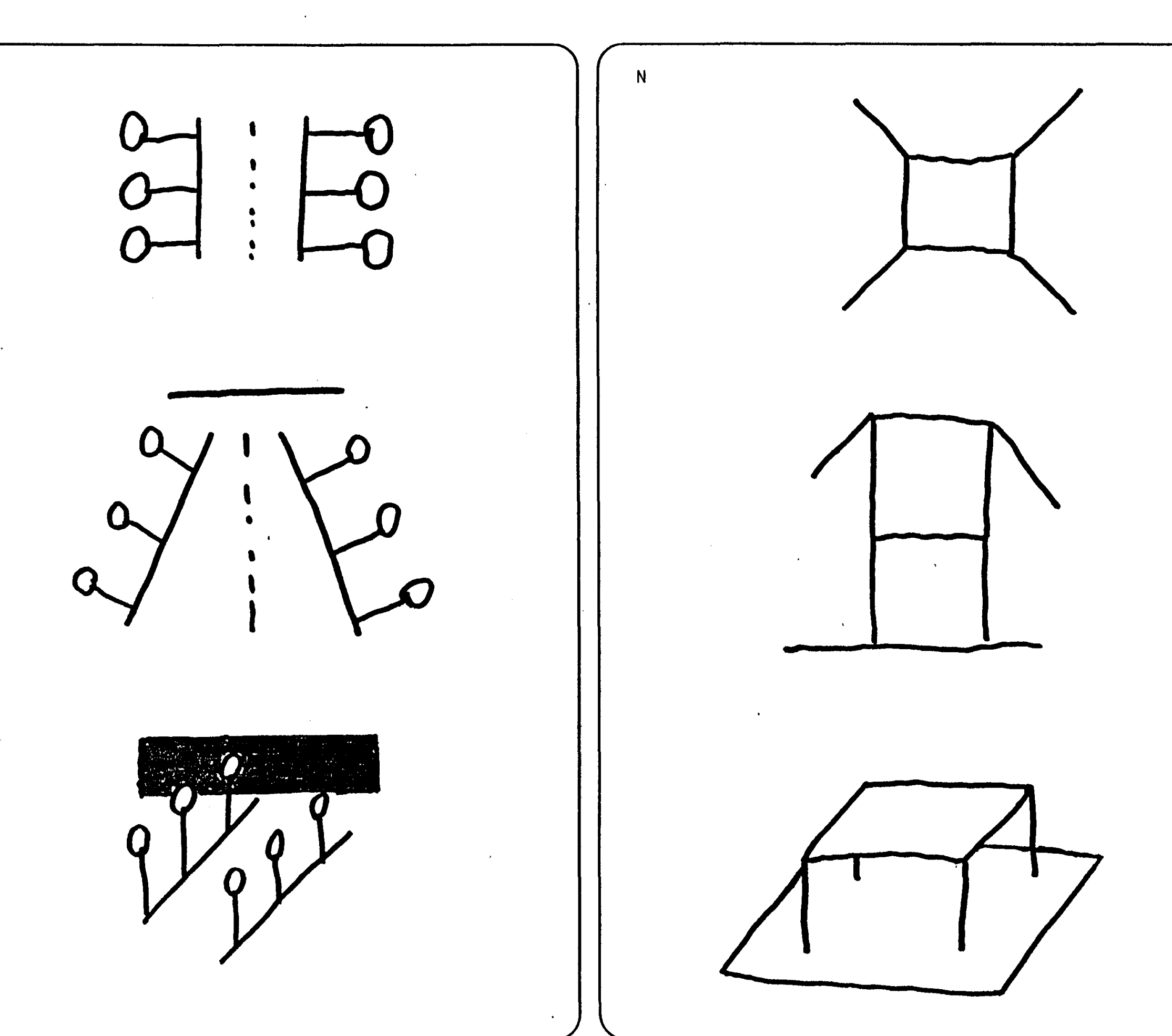




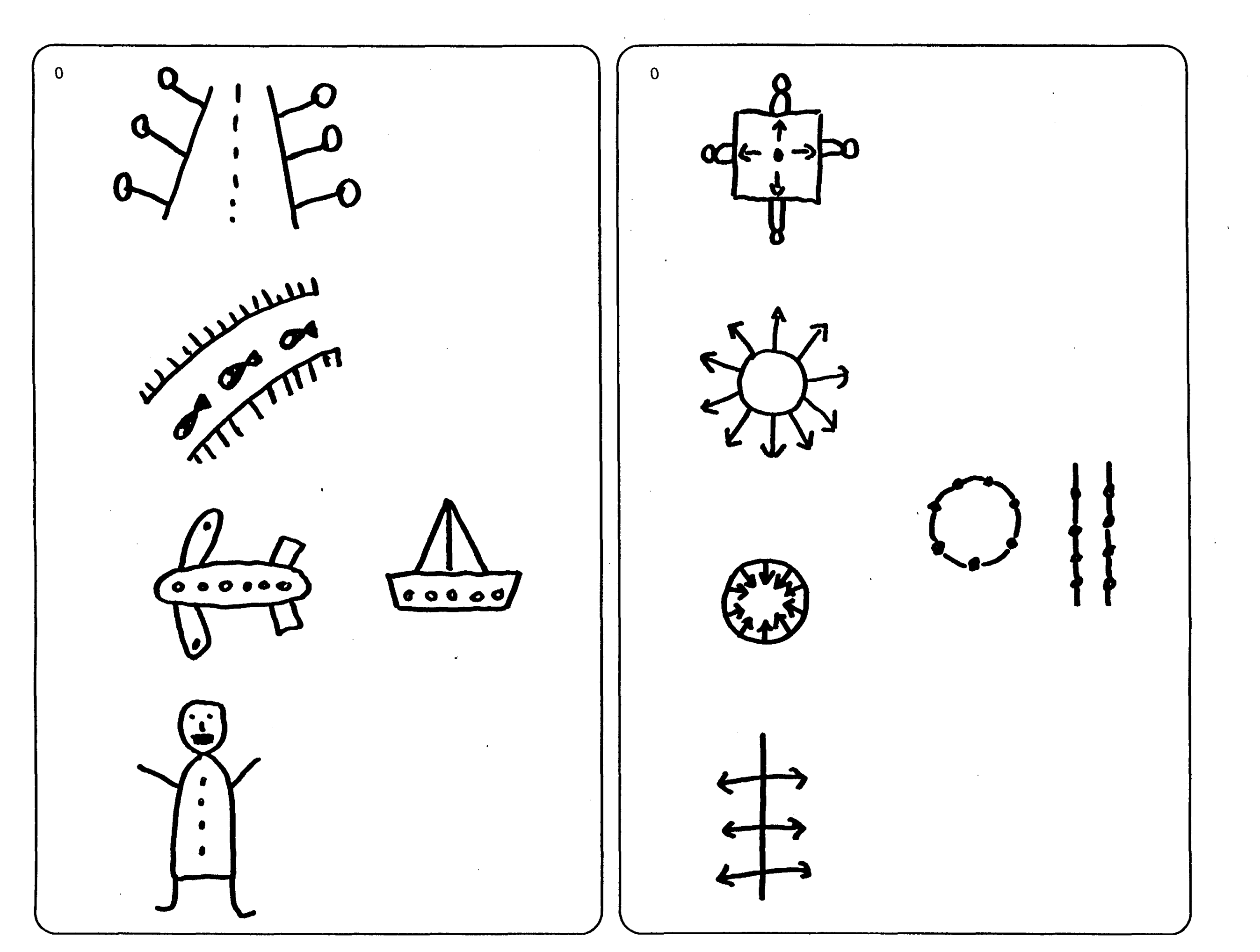




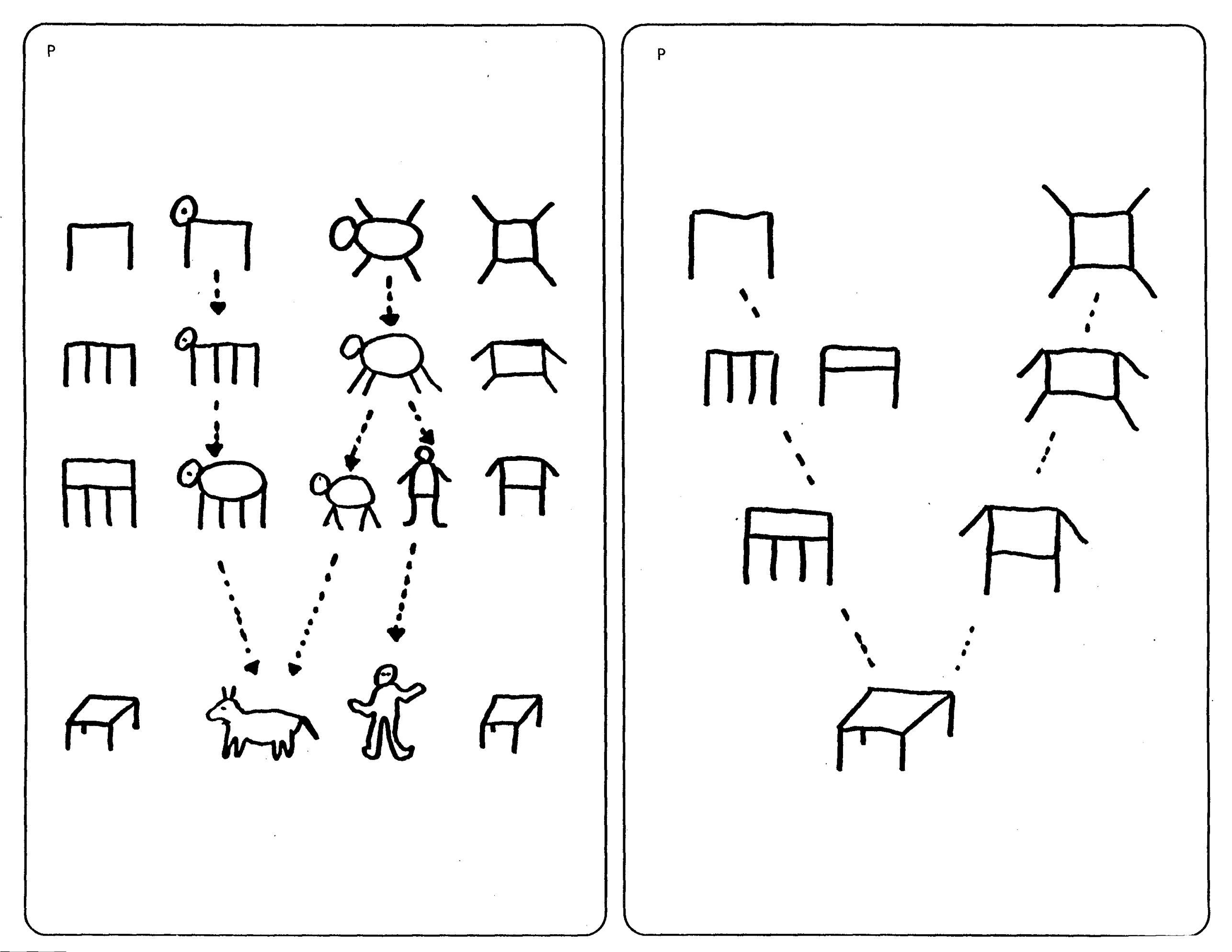




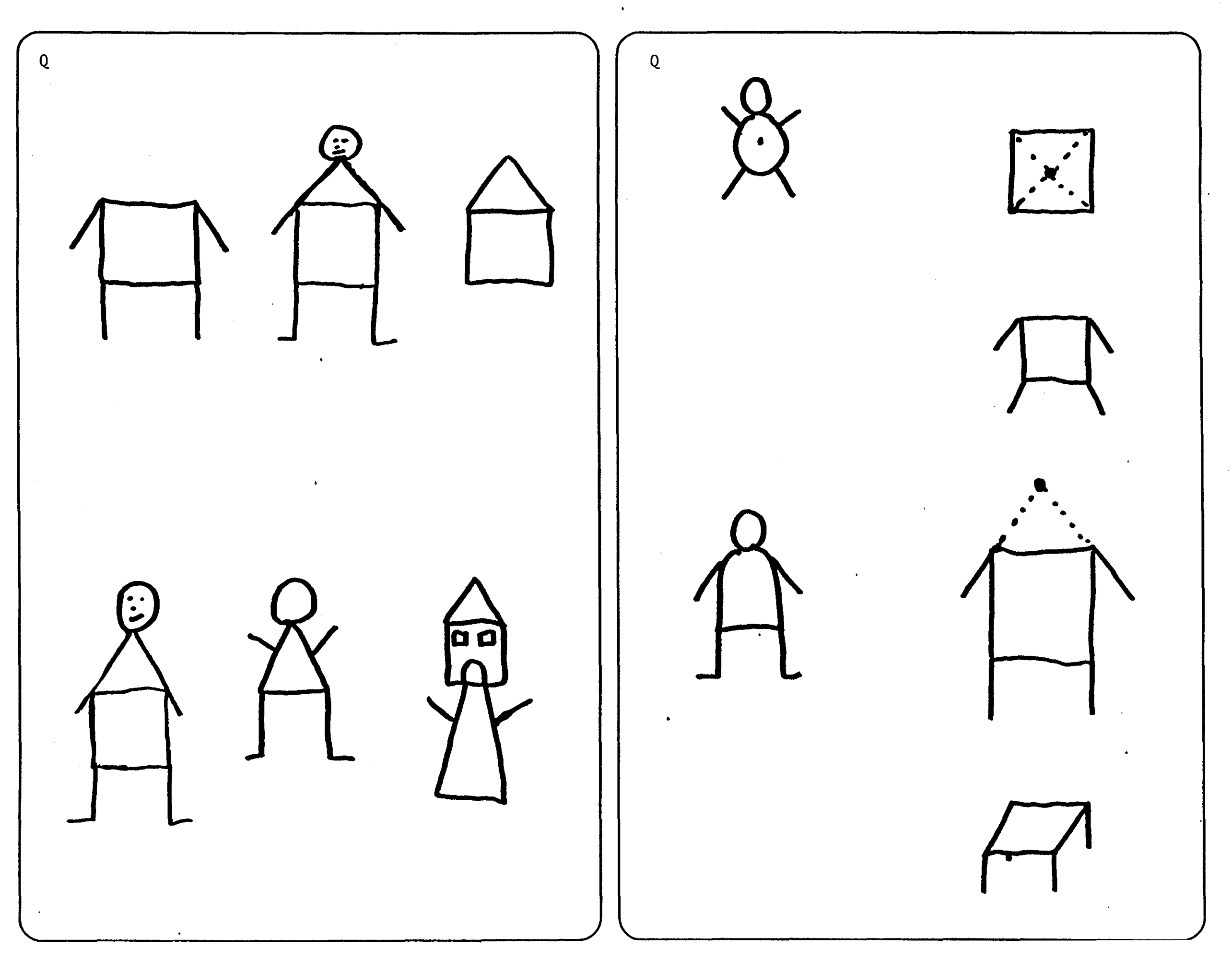




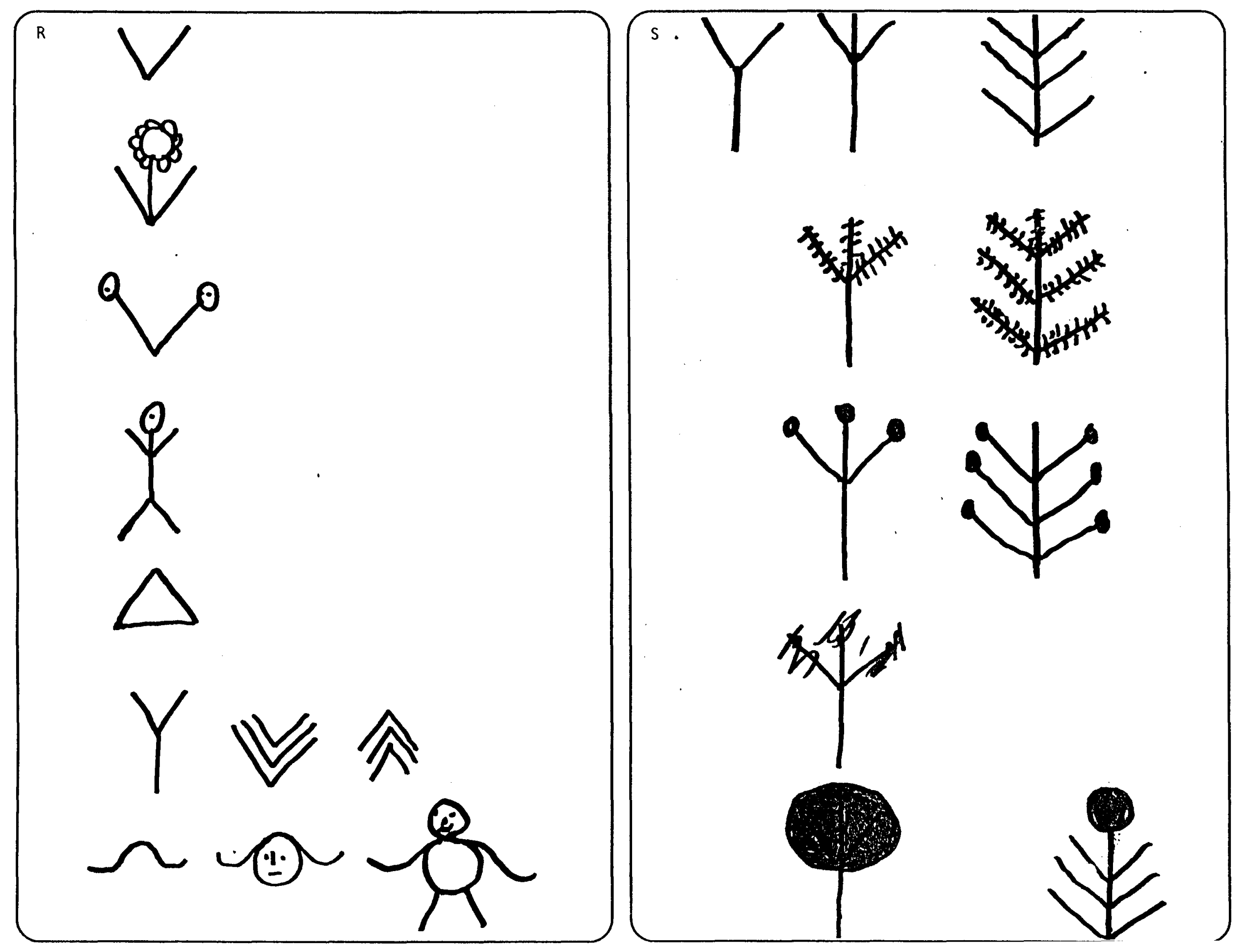




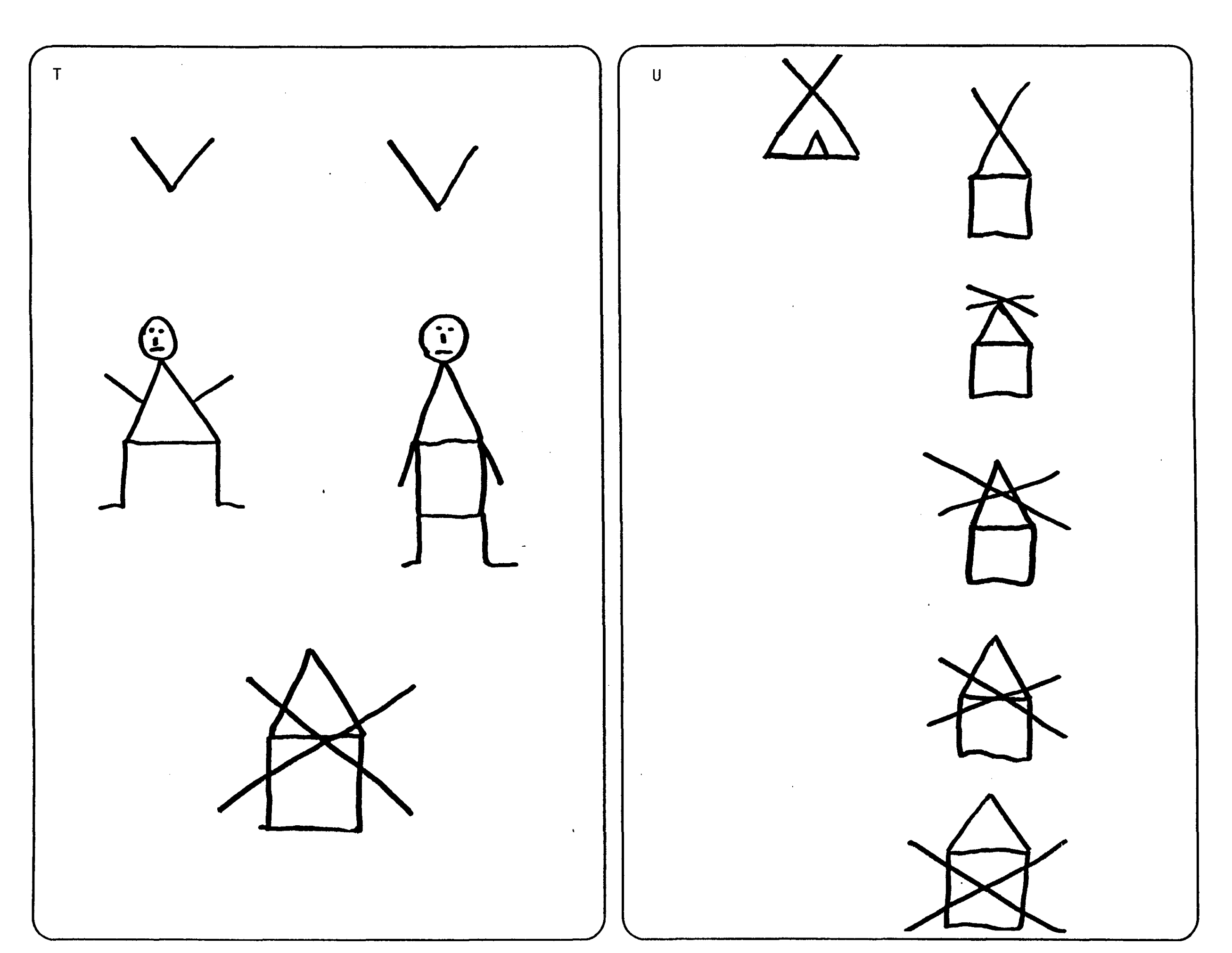




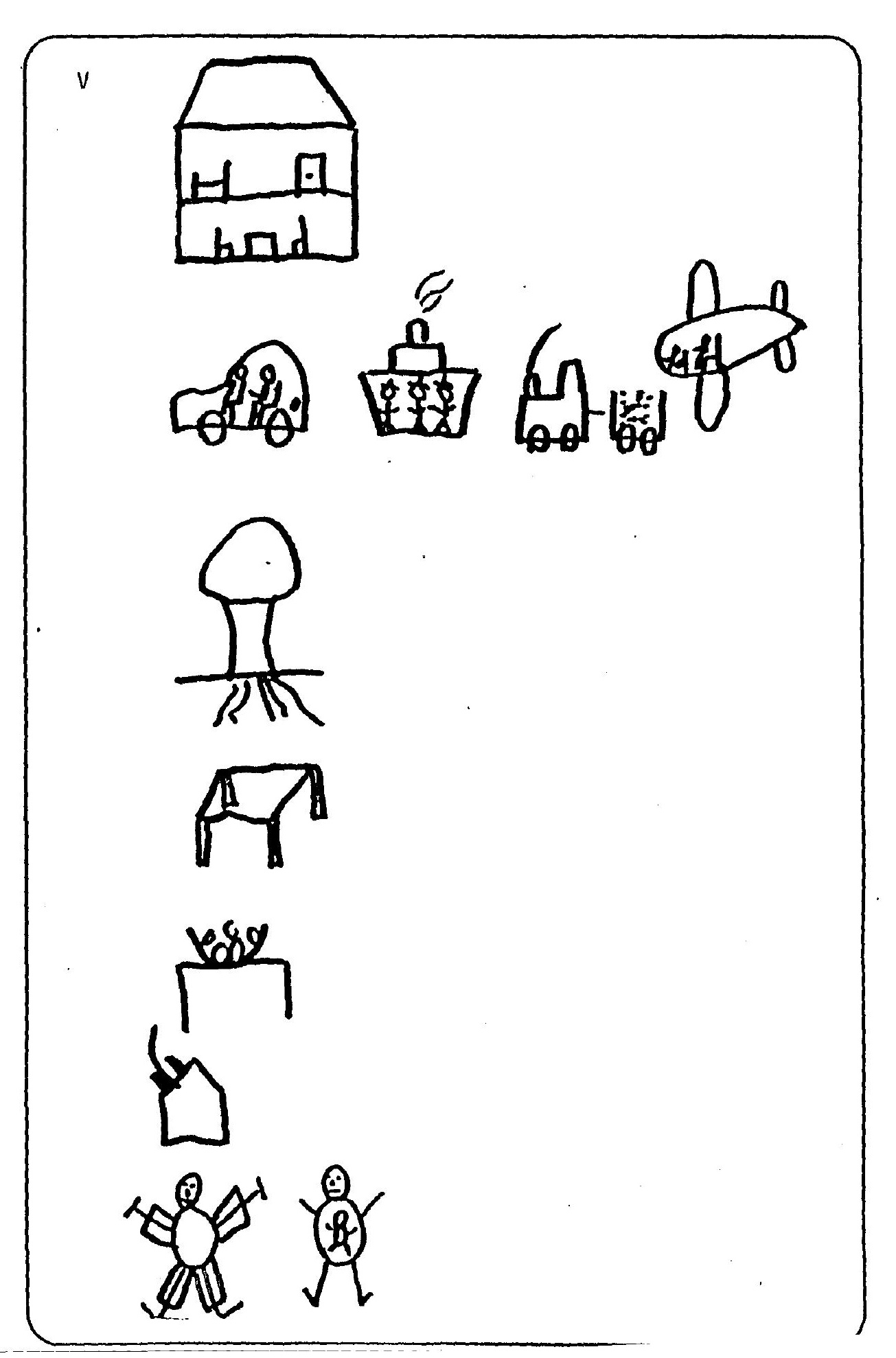




\begin{abstract}
ANNEXE III
Manifestations idéographiques

proposées par Clasca Ozinga
\end{abstract}




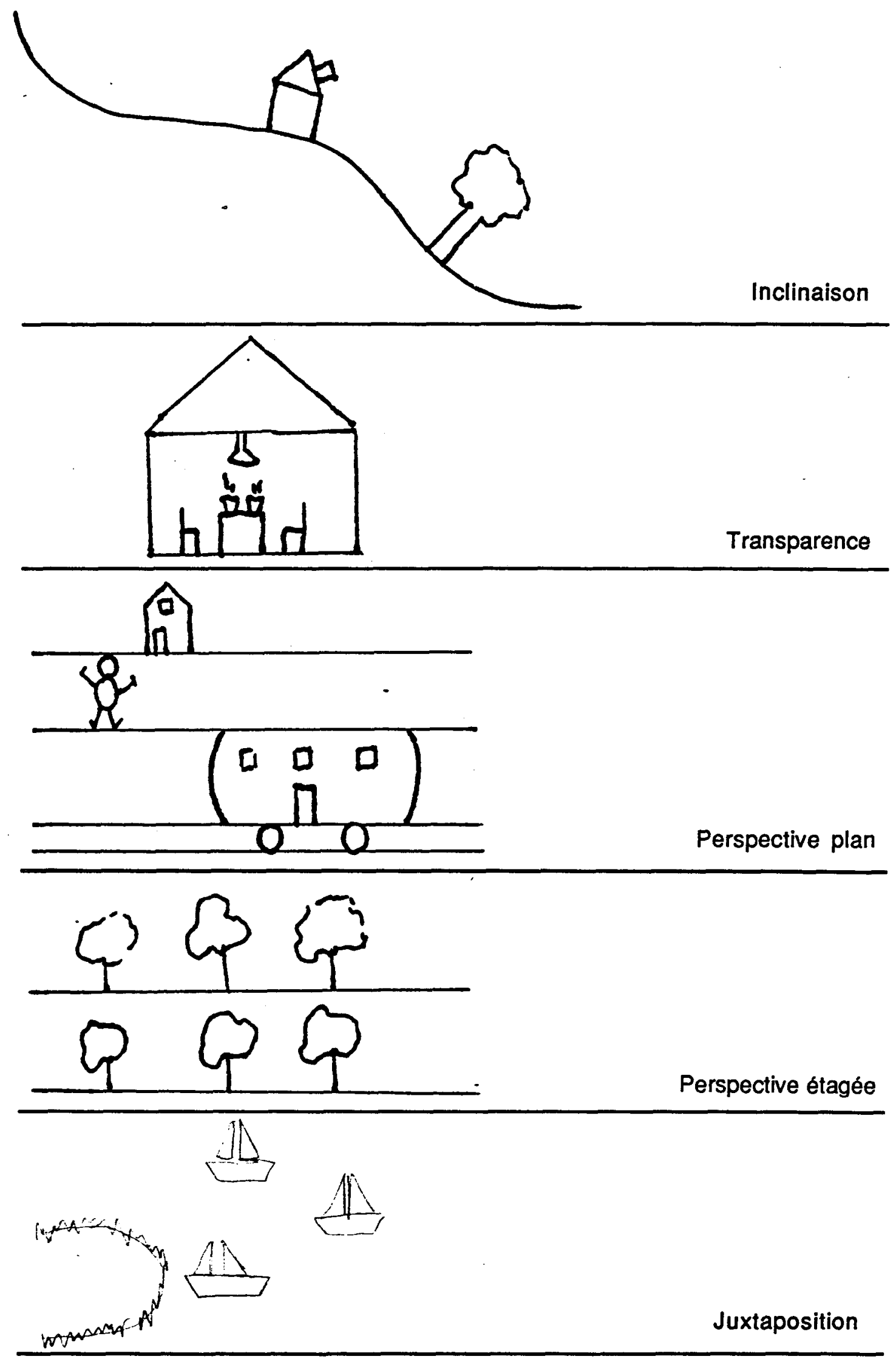




\begin{abstract}
ANNEXE IV
Reproduction du modèle table-palette de Stern adapté aux réalités d'une classe
\end{abstract}




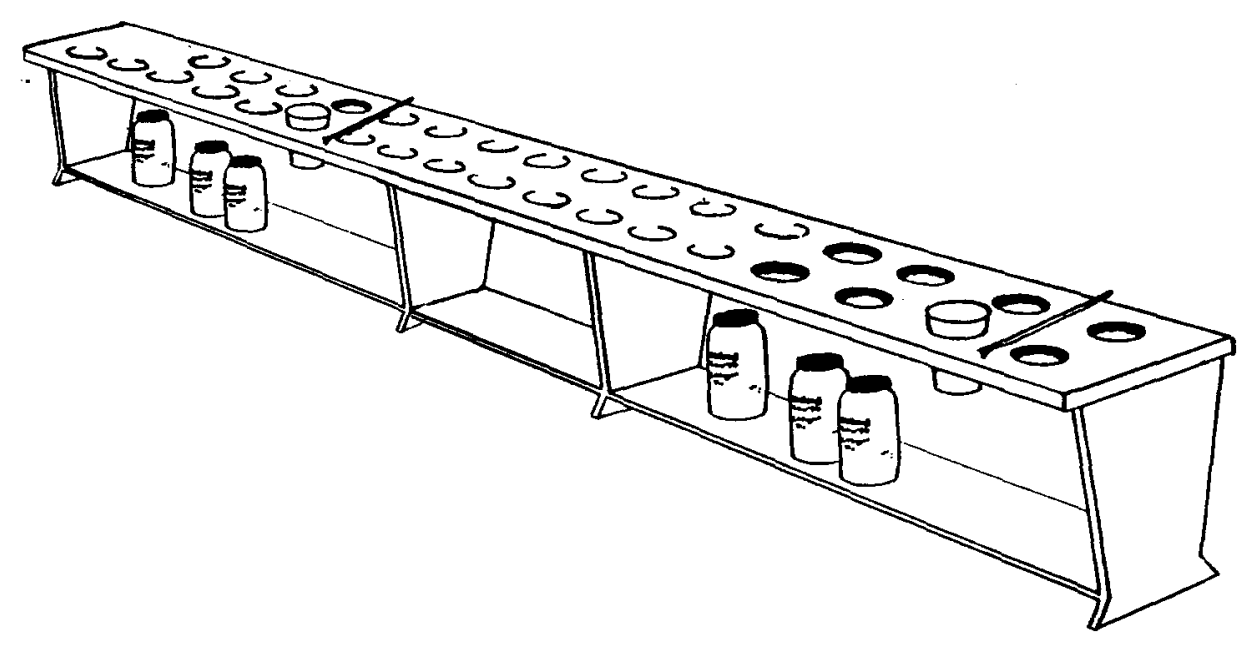

Table-palette de Stern.

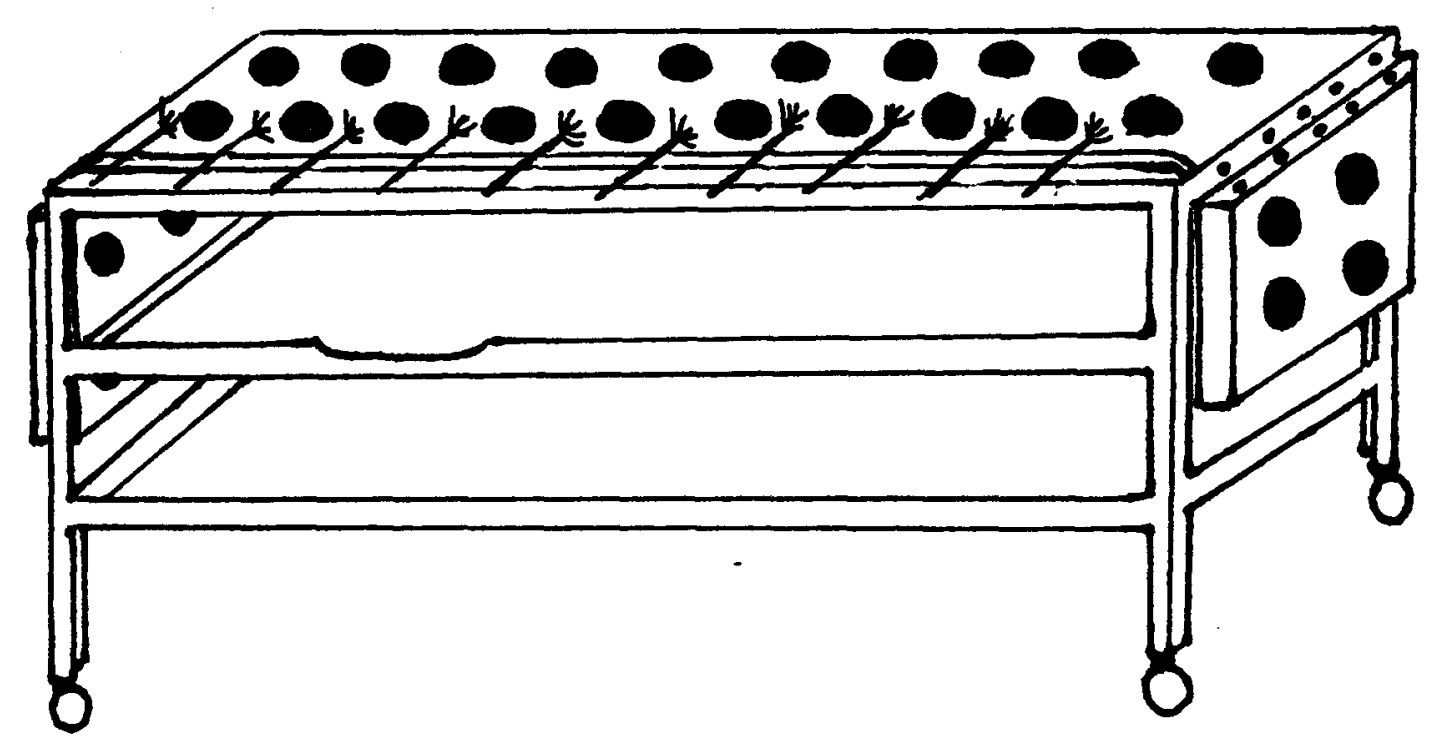

Table-palette adaptée. 
ANNEXE V

Reproduction du modèle de rangement pour la conservation des peintures 


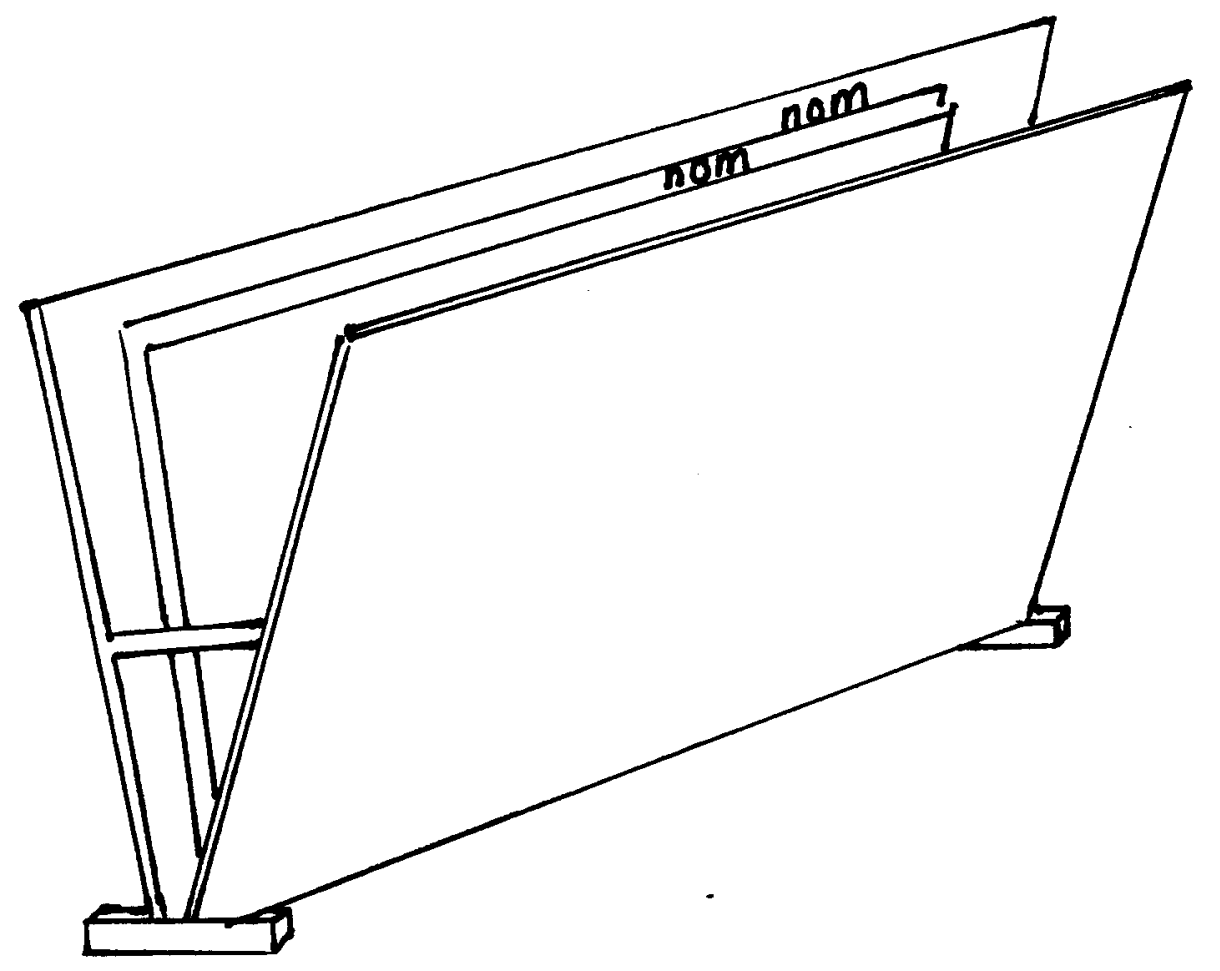




\begin{abstract}
ANNEXE VI
Reproduction des grilles utilisées pour l'analyse des peintures
\end{abstract}




\begin{tabular}{|l|}
\hline SOLEIL \\
\hline Cercle \\
\hline En coin \\
\hline En triangle \\
\hline Avec rayons \\
\hline Rayons seuls \\
\hline NUAGE \\
\hline Contour seul \\
\hline Contour $\neq$ contenu \\
\hline Contour=contenu \\
\hline Pas de contour \\
\hline Couleur réelle \\
\hline Couleur irréelle \\
\hline ARC EN ClEL \\
\hline Couleur réelle \\
\hline Couleur irréelle \\
\hline Superposition couleurs \\
\hline Couleur fondue \\
\hline Nombre de couleurs \\
\hline Répétition couleurs \\
\hline Autres \\
\hline
\end{tabular}

\begin{tabular}{|l|}
\hline Réel \\
\hline Irréel \\
\hline Tête \\
\hline Yeux \\
\hline Museau \\
\hline Gueule \\
\hline Dents \\
\hline Langue \\
\hline Cou \\
\hline Corps \\
\hline Pattes \\
\hline Griffes \\
\hline Queue \\
\hline $\begin{array}{l}\text { Représentation } \\
\text { de la peau }\end{array}$ \\
\hline Poils \\
\hline Plumes \\
\hline Écailles \\
\hline $\begin{array}{l}\text { Position réelle } \\
\text { quadrupédie }\end{array}$ \\
\hline Autres positions \\
\hline Couleur réelle \\
\hline Couleur irréelle \\
\hline Autres \\
\hline
\end{tabular}

\begin{tabular}{|l|}
\hline Articulation coude \\
\hline Articulation poignet \\
\hline Mains \\
\hline Doigts \\
\hline Ongles \\
\hline Présence mouvement \\
\hline Jambes \\
\hline Épaisseur jambe \\
\hline Articulation genou \\
\hline Articulation cheville \\
\hline Pieds \\
\hline Orteils \\
\hline Présence mouvement \\
\hline Costume identifiant \\
garçon ou fille \\
\hline Costume de rôle \\
\hline Coiffure \\
\hline Présence d'accessoire \\
\hline Costume avec motif \\
\hline Contour seul \\
\hline Contour $\neq$ contenu \\
\hline Contour=contenu \\
\hline Pas de contour \\
\hline Autres \\
\hline
\end{tabular}

\begin{tabular}{|l|}
\hline Première lignée \\
\hline Deuxième lignée \\
\hline Troisième lignée \\
\hline Figuration humaine \\
\hline Profil \\
\hline Tête \\
\hline Yeux \\
\hline Nez \\
\hline Bouche \\
\hline Oreilles \\
\hline Sourcils \\
\hline Cils \\
\hline Expresion visage \\
\hline Présence cheveux \\
\hline Couleurs cheveux \\
\hline Longueur cheveux \\
\hline Style raide \\
\hline Style frisé \\
\hline Style natté-gauffré \\
\hline Cou \\
\hline Épaules \\
\hline Corps \\
\hline Bras \\
\hline Épaisseur bras \\
\hline
\end{tabular}

Epaisseur bras 


\begin{tabular}{|l|}
\hline Noir d'ivoire \\
\hline Terre de Sienne \\
\hline Rouge vermillon \\
\hline Ocre jaune \\
\hline Jaune chrome clair \\
\hline Vert anglais foncé \\
\hline Bleu outremer \\
\hline Terre d'ombre brülée \\
\hline Jaune de chrome \\
\hline Blanc permanent \\
\hline Autres mélanges \\
\hline Contour seul \\
\hline Contourłcontenu \\
\hline Contour=contenu \\
\hline Pas de contour \\
\hline Couleur=objet \\
\hline Couleur=objet \\
\hline Présence nuance \\
\hline Couleurs superposées \\
\hline Ombre-lumière \\
\hline Présence texture \\
\hline Présence motif́ \\
\hline Autres \\
\hline
\end{tabular}

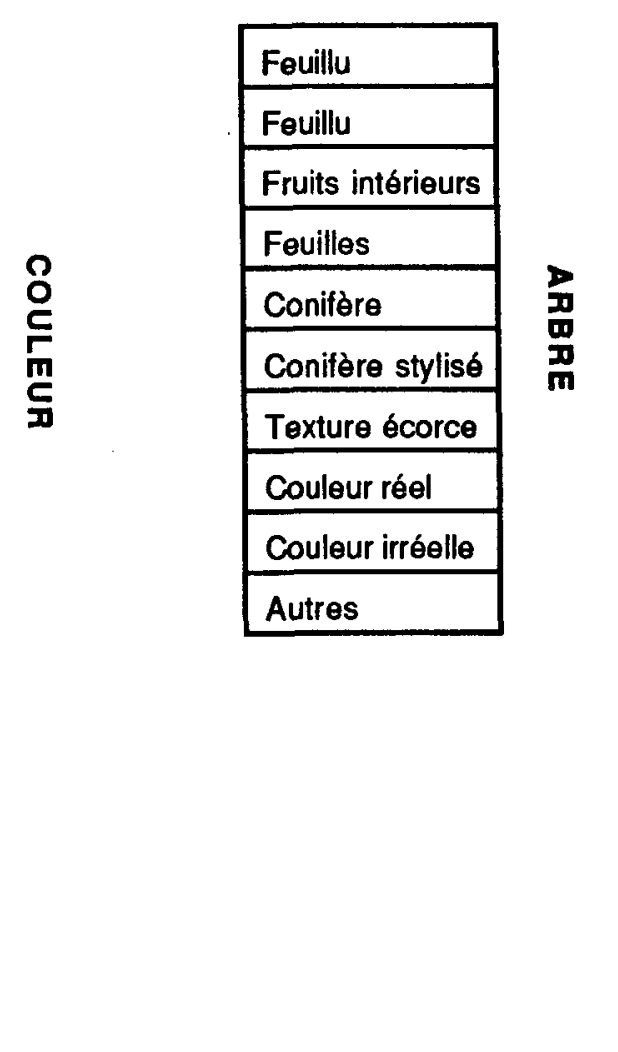

\begin{tabular}{|l|}
\hline Maison triangle \\
\hline Maison carrée \\
\hline Maison vue de face \\
\hline Maison face et profil \\
\hline Porte \\
\hline Poignée \\
\hline Fenêtre \\
\hline Carreau \\
\hline Fumée \\
\hline Rideau \\
\hline Cheminée \\
\hline Fumée \\
\hline Chemin \\
\hline Texture du mur extérieur \\
\hline Texture du chemin \\
\hline Autres \\
\hline
\end{tabular}




\begin{tabular}{|l|}
\hline Élements verticaux \\
\hline Suspension éléments \\
\hline Alignement éléments \\
\hline Alignement bas feuille \\
\hline Ligne du ciel \\
\hline Ligne de sol droite \\
\hline Ligne de sol courbe \\
\hline Espace sol et ciel \\
\hline Lignesol reiointciel \\
\hline Ordre des éléments \\
\hline Forme close-contenant \\
\hline Remplissage entier \\
\hline Encadrement \\
\hline Exemplarité \\
\hline Exagération objet \\
\hline Inclinaison \\
\hline Répétition automatigue \\
\hline Juxtaposition \\
\hline Transparence \\
\hline Rabattement \\
\hline Symétrie \\
\hline Perspective plan \\
\hline Perspective étagée \\
\hline Perspective vol oiseau \\
\hline Perspective polygone \\
\hline Diff. séq.-même endroit \\
\hline Diff. act.-temps diff. \\
\hline Objets éloignés réduits \\
\hline
\end{tabular}




\section{ANNEXE VII}

Le nombre de productions réalisées

par chacun des pré-adolescents 


\begin{tabular}{|c|c|}
\hline $\begin{array}{c}\text { Identification } \\
\text { de l'enfant }\end{array}$ & Nombre de dessins \\
\hline $\begin{array}{r}1 \\
2 \\
3 \\
4 \\
5 \\
6 \\
7 \\
8 \\
9 \\
10 \\
11 \\
12 \\
13 \\
14 \\
15 \\
16 \\
17 \\
18 \\
19 \\
20 \\
21 \\
22 \\
23 \\
24 \\
25 \\
26\end{array}$ & $\begin{array}{l}15 \\
13 \\
20 \\
14 \\
17 \\
13 \\
13 \\
14 \\
13 \\
17 \\
19 \\
15 \\
13 \\
12 \\
15 \\
16 \\
16 \\
14 \\
13 \\
21 \\
24 \\
13 \\
14 \\
12 \\
20 \\
13\end{array}$ \\
\hline
\end{tabular}

